Portland State University

PDXScholar

\title{
Introduction to Data Science for Transportation Researchers, Planners, and Engineers
}

Liming Wang

Portland State University, limwang@pdx.edu

Follow this and additional works at: https://pdxscholar.library.pdx.edu/trec_reports

Part of the Transportation Commons, Urban Studies Commons, and the Urban Studies and Planning Commons

Let us know how access to this document benefits you.

\section{Recommended Citation}

Wang, Liming. Introduction to Data Science for Transportation Researchers, Planners, and Engineers. NITC-ED-854. Portland, OR: Transportation Research and Education Center (TREC), 2018. https://doi.org/ 10.15760/trec.192

This Report is brought to you for free and open access. It has been accepted for inclusion in TREC Final Reports by an authorized administrator of PDXScholar. Please contact us if we can make this document more accessible: pdxscholar@pdx.edu. 


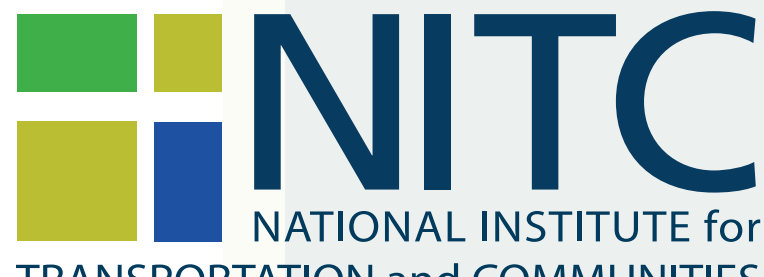

TRANSPORTATION and COMMUNITIES

FINAL REPORT

Introduction to Data Science for Transportation Researchers, Planners, and Engineers

NITC-ED-854 January 2018

NITC is a U.S. Department of Transportation

national university transportation center.

H:H TREC 



\title{
INTRODUCTION TO DATA SCIENCE FOR TRANSPORTATION RESEARCHERS, PLANNERS, AND ENGINEERS
}

\author{
Final Report
}

NITC-ED-854

by

Liming Wang

Portland State University

for

National Institute for Transportation and Communities (NITC)

P.O. Box 751

Portland, OR 97207
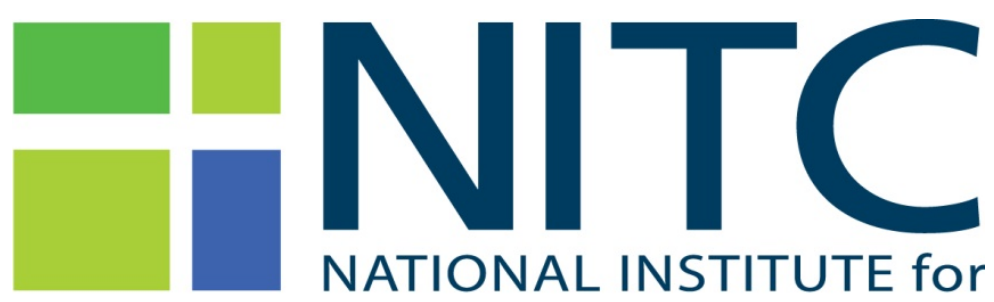

TRANSPORTATION and COMMUNITIES

January 2017 



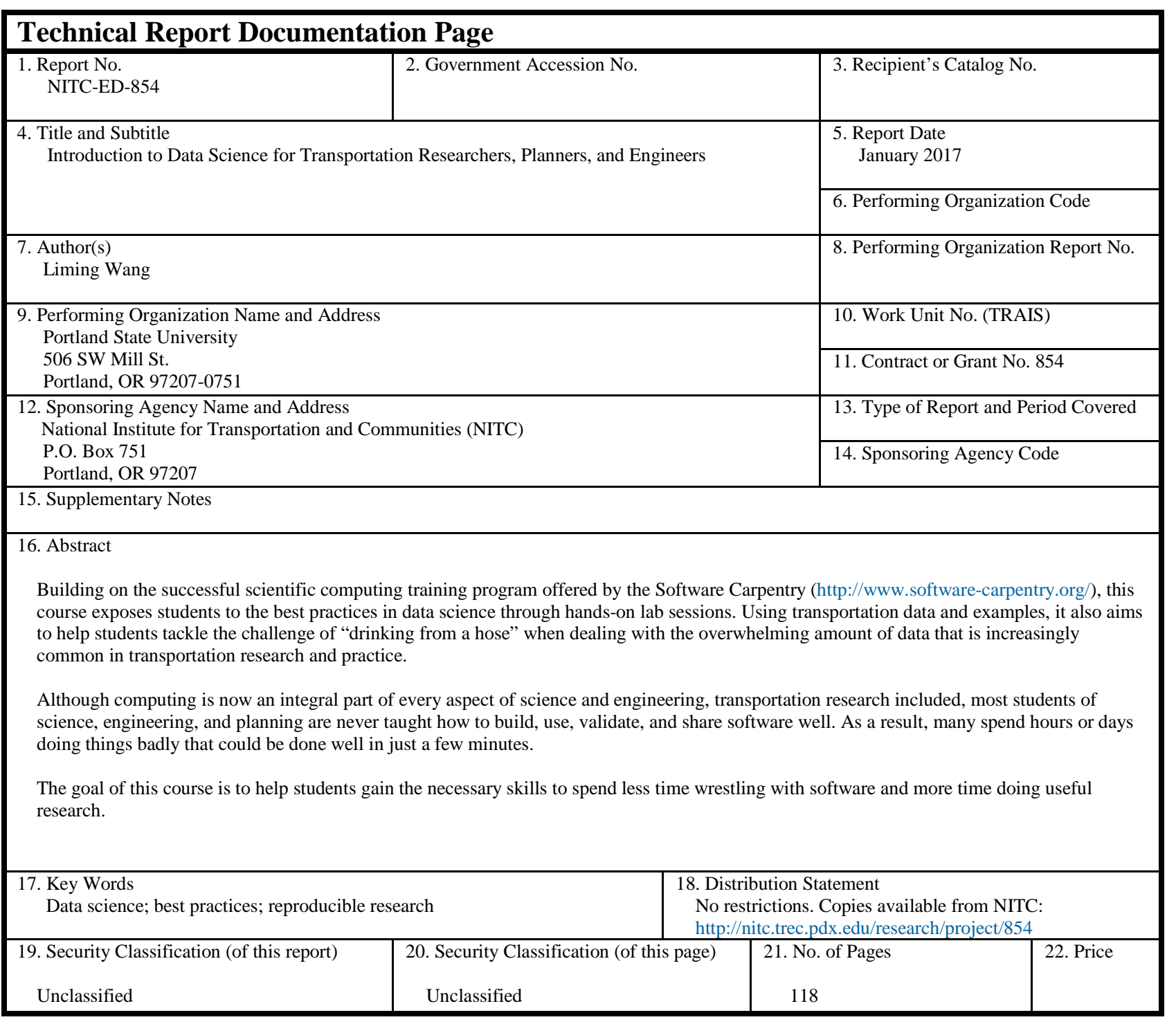




\section{ACKNOWLEDGMENTS}

This course was developed with financial support from the National Institute of Transportation and Communities under grant number 854.

I am grateful to the participants of the 2017 summer course for their valuable feedback. The support of TREC staff, in particular, Lisa Patterson and Eva-Maria Muecke, is greatly appreciated. Jamaal Green and Dillon Mahmoudi, both graduate students at Portland State University, have given me valuable feedback and assistance in the process of developing the course.

Parts of the course materials have been adapted from the following sources:

- R for Data Science by Hadley Wickham

- Software Carpentry workshop lessons

- UBC Stat 545 by Professor Jenny Bryan at UBC

- NEU 5110 Introduction to Data Science by Professor Jan Vitek

The write-up and website are powered by the bookdown package and GitHub.

\section{DISCLAIMER}

The contents of this report reflect the views of the authors, who are solely responsible for the facts and the accuracy of the material and information presented herein. This document is disseminated under the sponsorship of the U.S. Department of Transportation University Transportation Centers Program in the interest of information exchange. The U.S. Government assumes no liability for the contents or use thereof. The contents do not necessarily reflect the official views of the U.S. Government. This report does not constitute a standard, specification or regulation.

\section{RECOMMENDED CITATION}

Wang, Liming. Introduction to Data Science for Transportation Researchers, Planners, and Engineers. NITC-ED-854. Portland, OR: Transportation Research and Education Center (TREC), 2018. 


\section{TABLE OF CONTENTS}

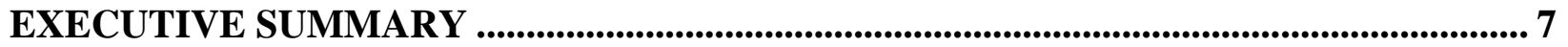

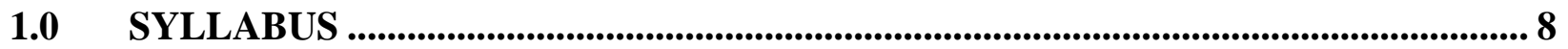

1.1 FORMAT, SOFTWARE AND HARDWARE...................................................... 8

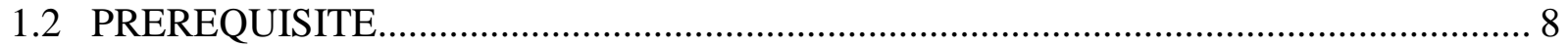

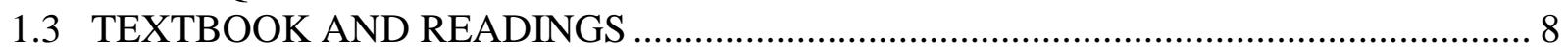

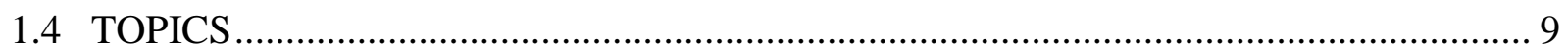

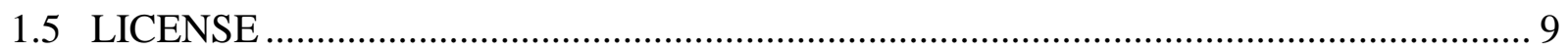

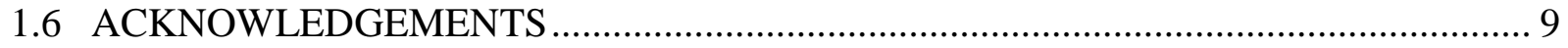

2.0 OVERVIEW AND INTRODUCTION …....................................................................... 11

2.1 PART I: DATA SCIENCE BEST PRACTICES ....................................................... 11

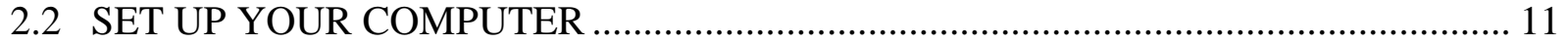

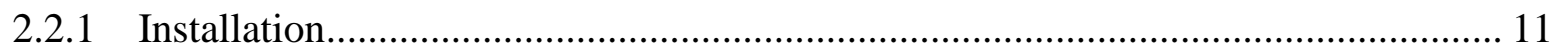

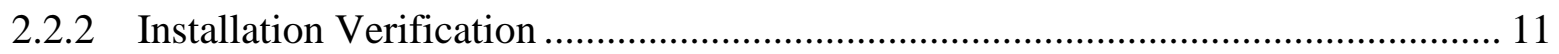

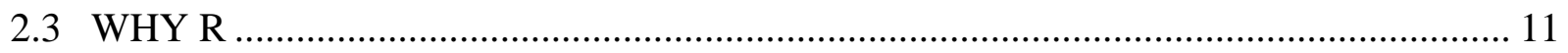

2.4 INTRODUCTION TO R AND RSTUDIO .............................................................. 11

2.4.1 Project Organization in RStudio ………….................................................. 12

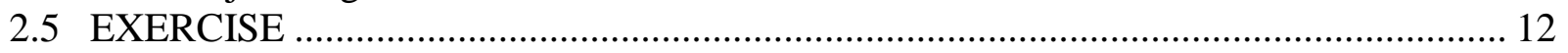

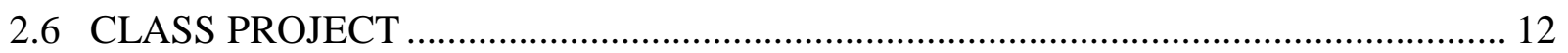

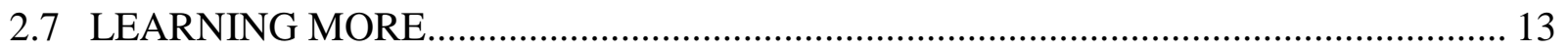

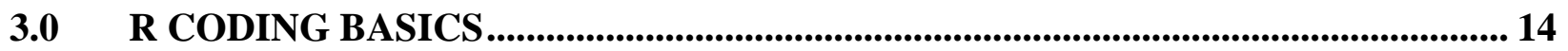

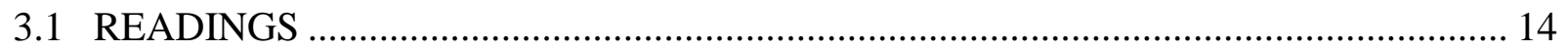

3.2 BEST PRACTICES FOR DATA SCIENCE ……………......................................... 14

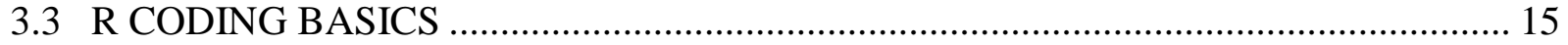

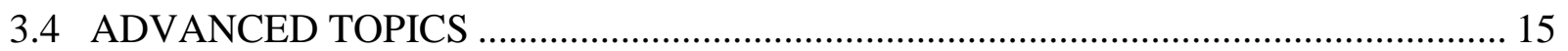

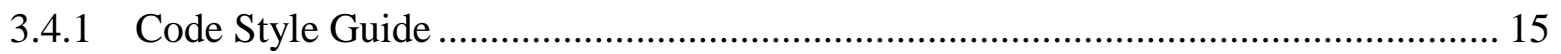

3.4.2 R Command-Line Program................................................................................... 15

3.4.3 Debugging with RStudio.......................................................................................... 17

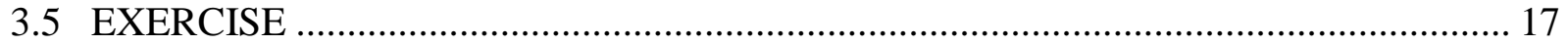

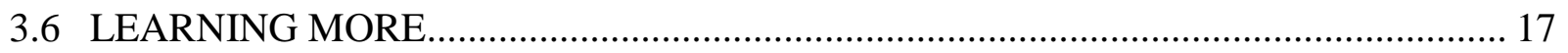

4.0 VERSION CONTROL WITH GIT ................................................................. 18

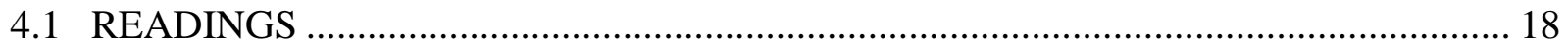

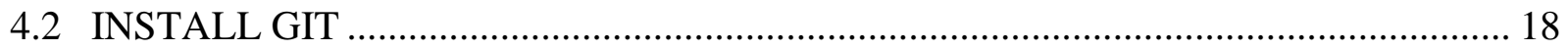

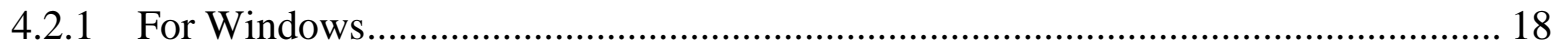

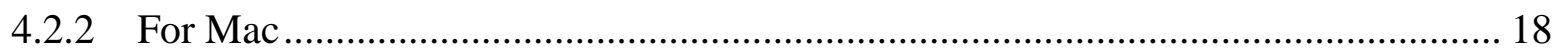

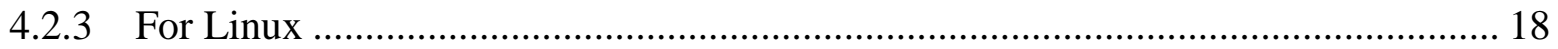

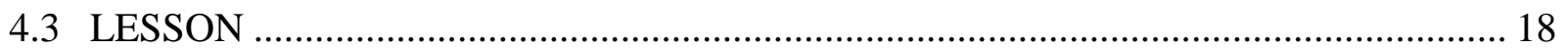

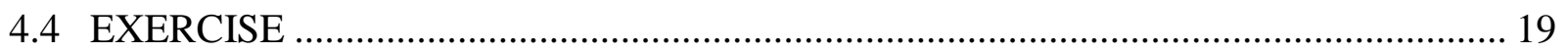

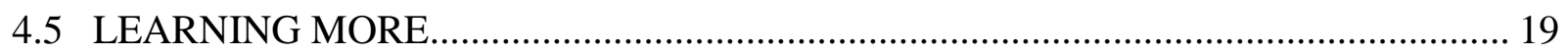

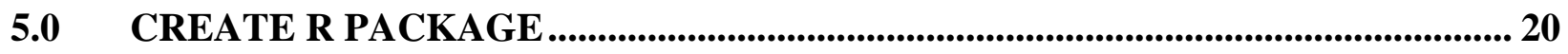

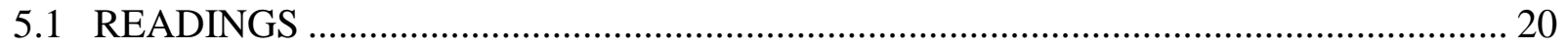




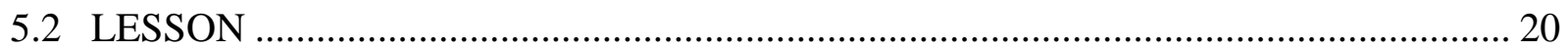

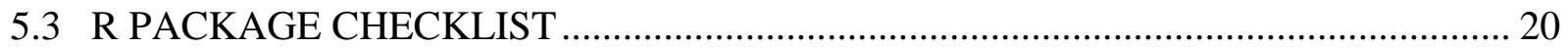

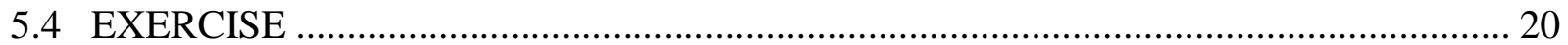

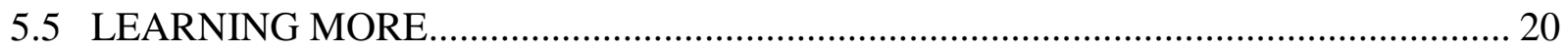

6.0 R MARKDOWN ............................................................................................................. 21

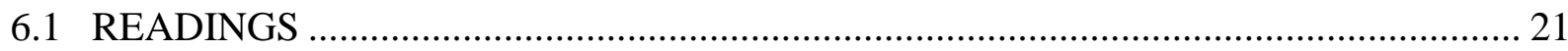

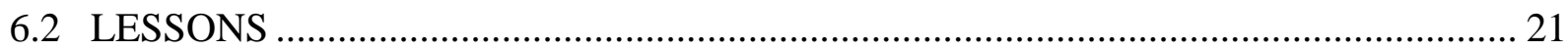

6.2.1 Formatting Text in Markdown................................................................................ 21

6.2.2 Use knitr to Produce a Report ................................................................................... 22

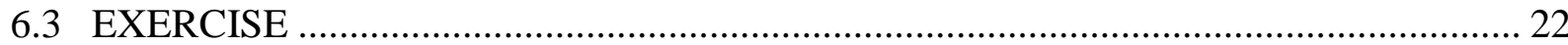

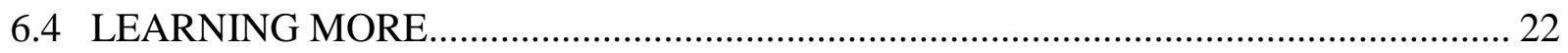

7.0 PART II: OVERVIEW AND INTRODUCTION........................................................... 23

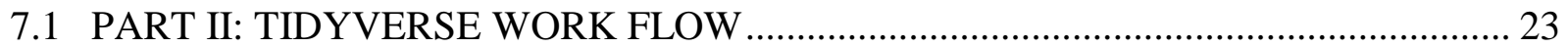

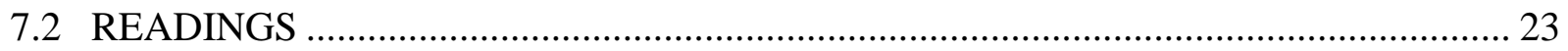

7.3 THE TIDYVERSE WORKFLOW …………………................................................ 23

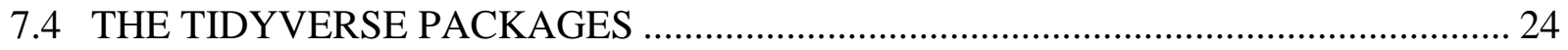

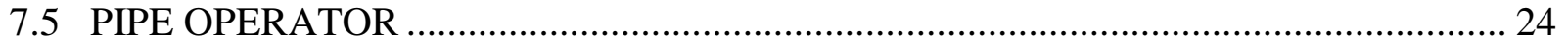

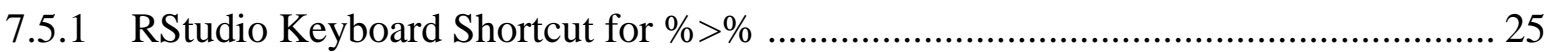

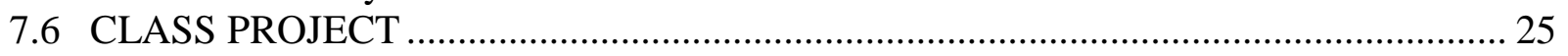

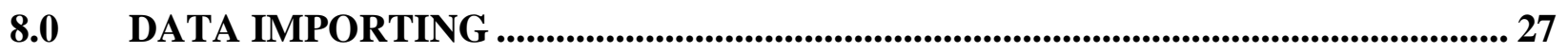

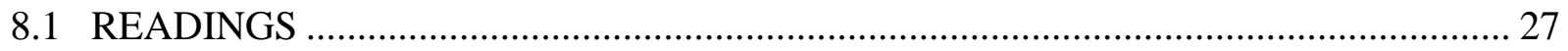

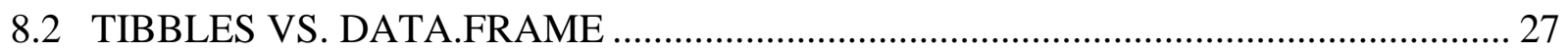

8.2.1 Data Frames are Awesome ………………….......................................................... 27

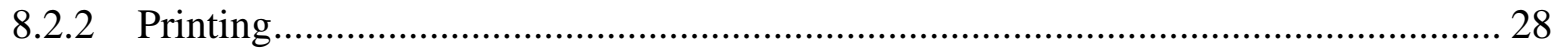

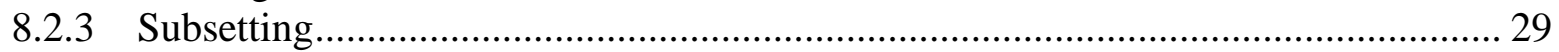

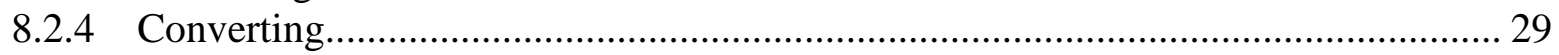

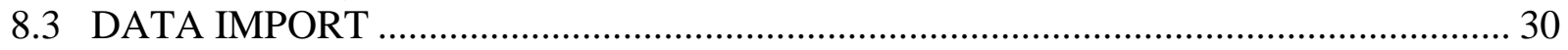

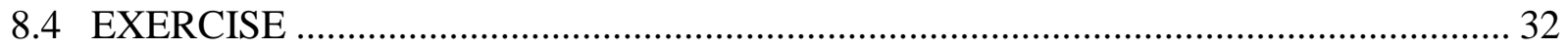

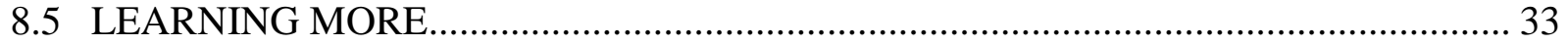

9.0 TIDY DATA ...................................................................................................... 34

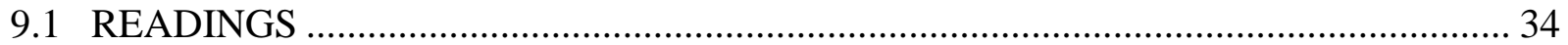

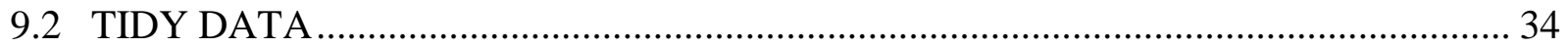

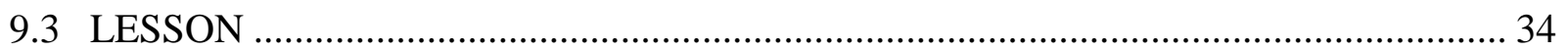

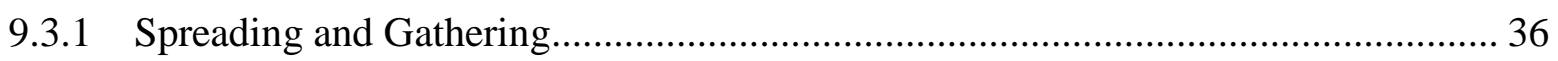

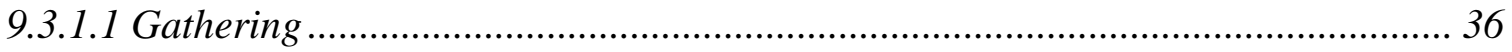

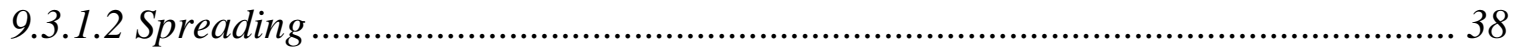

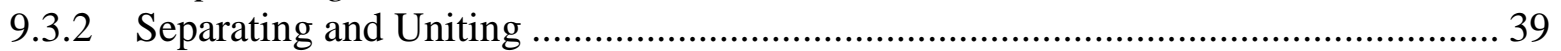

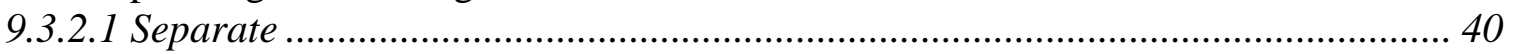

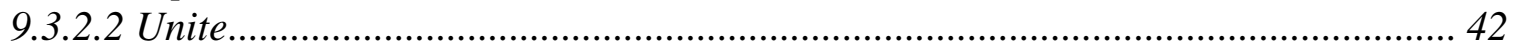

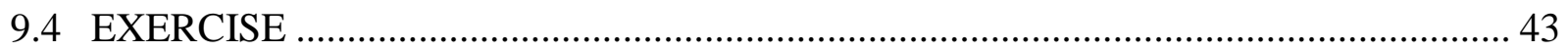

9.5 LEARNING MORE............................................................................................ 44

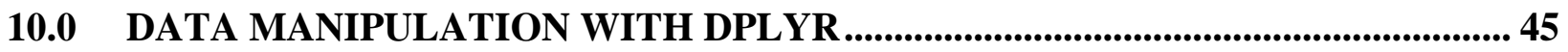

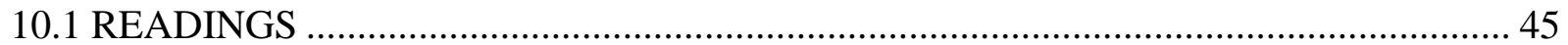

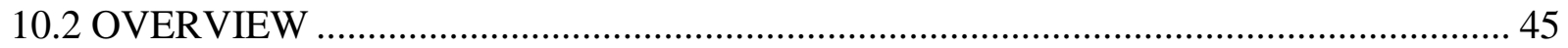

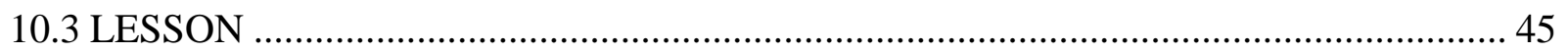




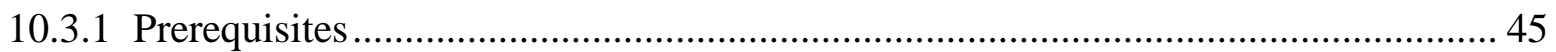

10.3.2 Use filter() to Subset Data Row-wise. ...................................................................... 45

10.3.3 Use select() to Subset the Data on Variables or Columns......................................... 46

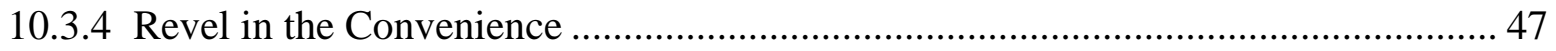

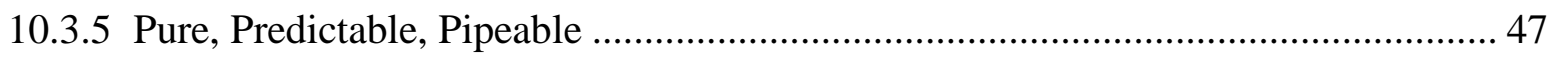

10.3.6 Use mutate() to Add New Variables .......................................................................... 48

10.3.7 Use arrange() to Row-order Data in a Principled Way ............................................. 51

10.3.8 Use rename() to Rename Variables ............................................................................. 52

10.3.9 select() Can Rename and Reposition Variables............................................................ 53

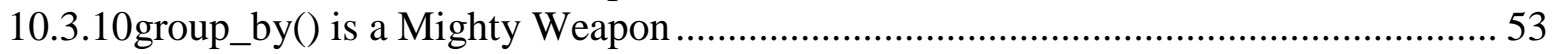

10.3.10.1 Counting Things Up ................................................................................ 54

10.3.10.2 General Summarization ....................................................................... 55

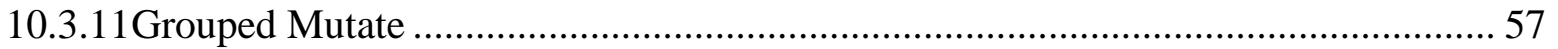

10.3.11.1 Computing With Group-wise Summaries.............................................. 57

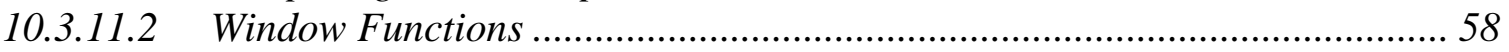

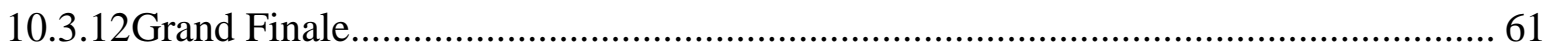

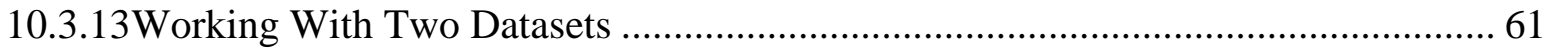

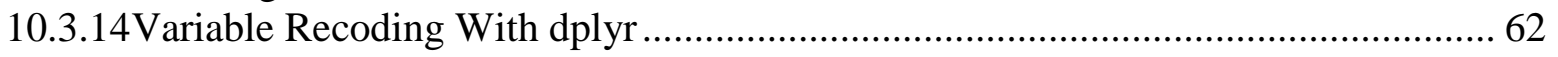

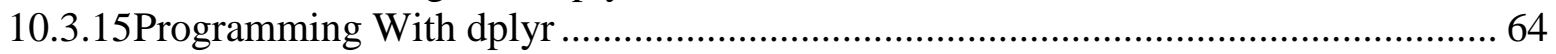

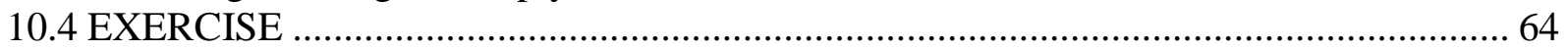

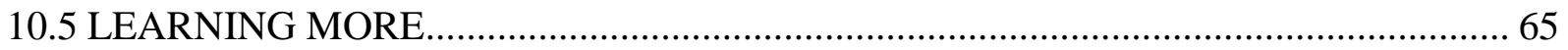

11.0 DATA VISUALIZATION WITH GGPLOT2 …........................................................66

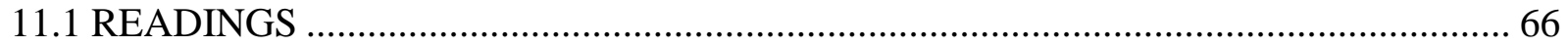

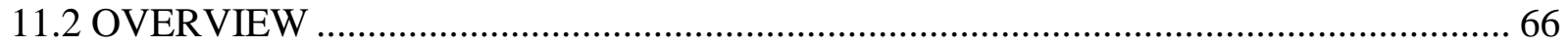

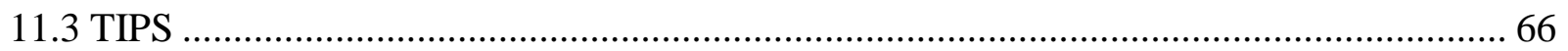

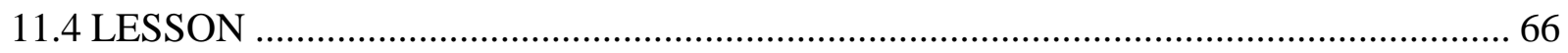

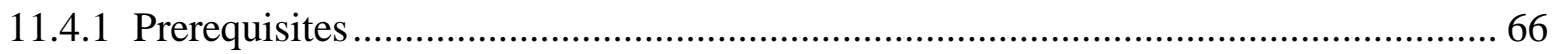

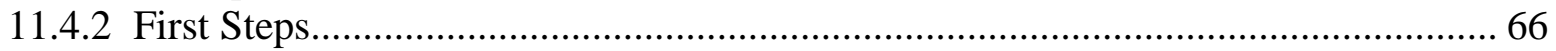

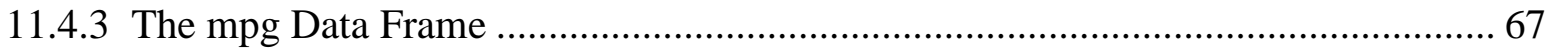

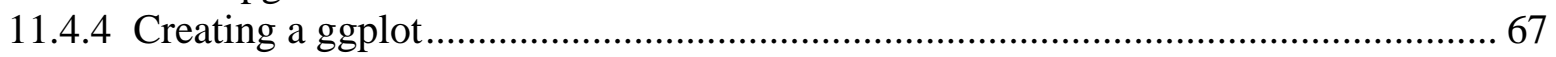

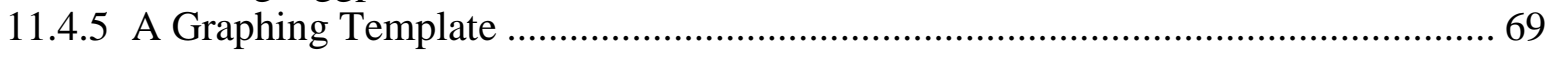

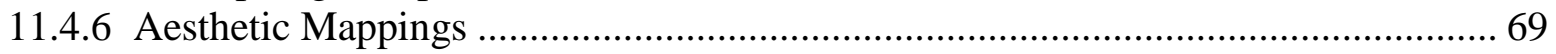

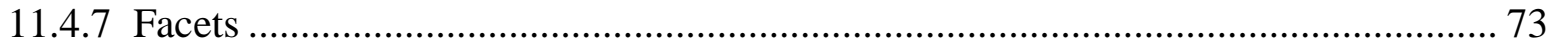

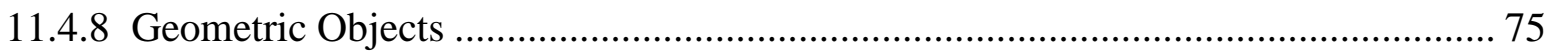

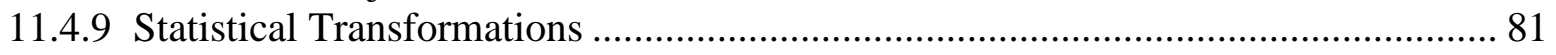

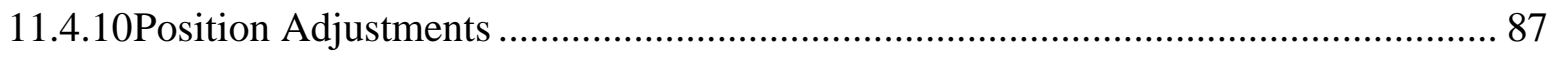

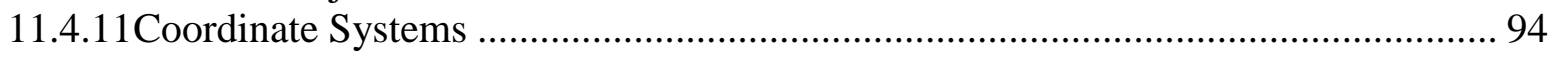

11.4.12The Layered Grammar of Graphics ......................................................................... 97

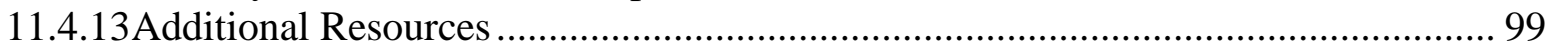

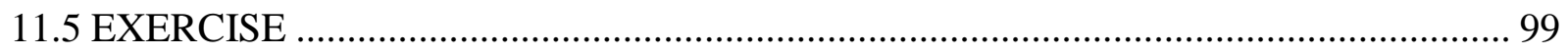

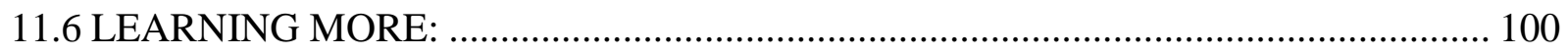

12.0 SPLIT-APPLY-COMBINE PATTERN IN DATA MODELING ........................... 101

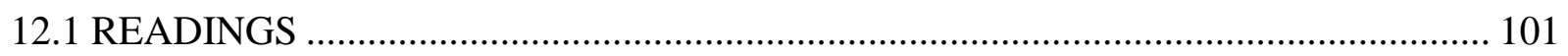

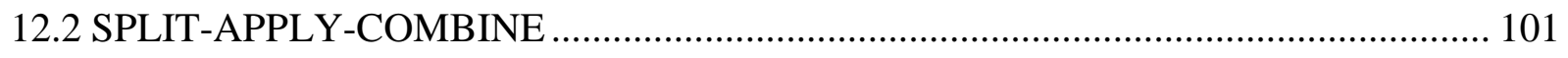

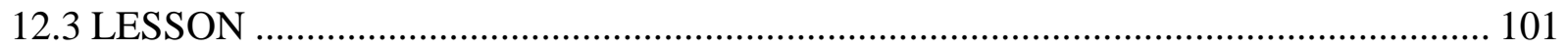

12.3.1 Think Before You Create Excerpts of Your Data ..................................................... 101 


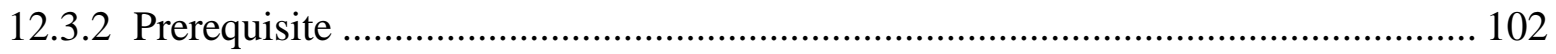

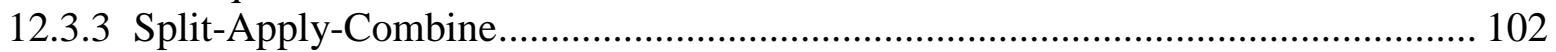

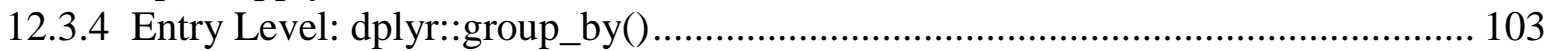

12.3.5 General Approach: Nesting....................................................................................... 103

12.3.6 Apply a Function purrr::map() and mutate() ........................................................ 105

12.3.7 Always Have an Exit Strategy .......................................................................... 107

12.3.8 Simplify and Combine ................................................................................. 107

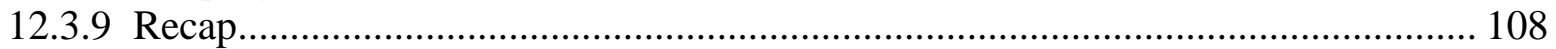

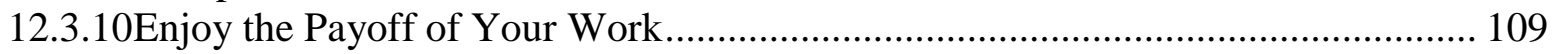

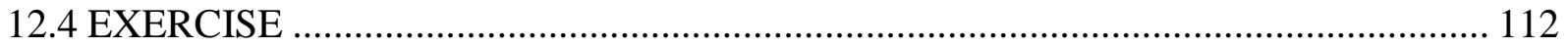

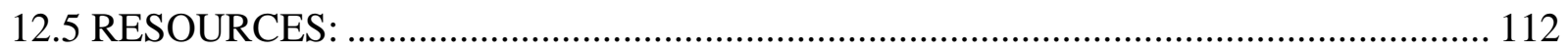

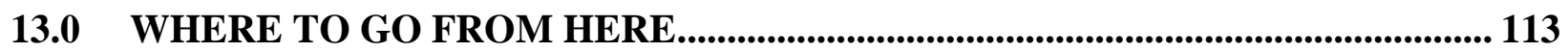

13.1 NOTICEABLE FEATURES NOT COVERED ………........................................... 113

13.1.1 Automate Testing Your Code ............................................................................ 113

13.1.2 Automate Your Workflow ……………………................................................. 113

13.1.3 Develop Interactive Apps With Shiny ………….................................................... 113

13.2 WHERE TO FIND HELP................................................................................. 114

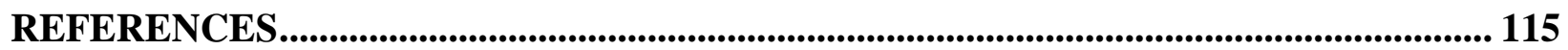

\section{LIST OF TABLES}

Table 9.1 Bike counts by day of week and bridge (1st format) ................................................. 43

Table 9.2 Bike counts by day of week and bridge (2nd format).................................................... 43

\section{LIST OF FIGURES}

Figure 9.1 Gathering table4 into a tidy form ............................................................................. 37

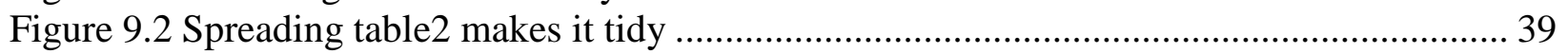

Figure 9.3 Separating table3 makes it tidy.............................................................................. 41

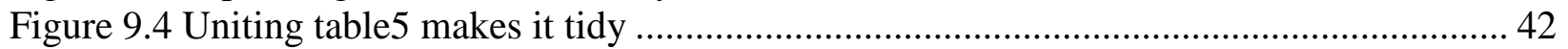

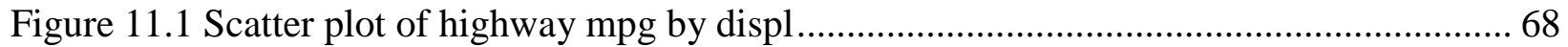

Figure 11.2 Scatter plot with some cars highlighted in red ........................................................ 69

Figure 11.3 Scatter plot of highway mpg by displ, with class of cars differentiated using color. 70 Figure 11.4 Scatter plot of highway mpg by displ, with class of cars differentiated using size... 71

Figure 11.4 Scatter plot of highway mpg by displ, with class of cars differentiated using size... 72

Figure 11.5 Scatter plot with color aesthetic manually set to blue ……………………………..... 73

Figure 11.6 Scatter plots of highway mpg by displ, faceted by class ............................................ 74

Figure 11.6 Scatter plots of highway mpg by displ, faceted by class ............................................. 75

Figure 11.7 Scatter plot and smooth curve of highway mpg by displ ............................................ 76

Figure 11.8 Smooth curves of highway mpg by displ, grouped by drv ........................................ 78

Figure 11.9 Scatter plot and smooth curves of highway mpg by displ, grouped by drv ............... 79

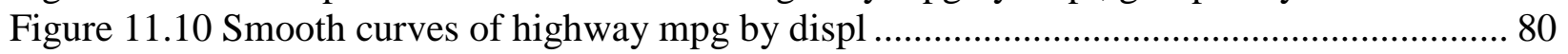

Figure 11.11 Scatter plot of highway mpg by displ super-imposed with stat_smooth................... 81

Figure 11.12 Bar chart of diamonds by cut................................................................................. 82 


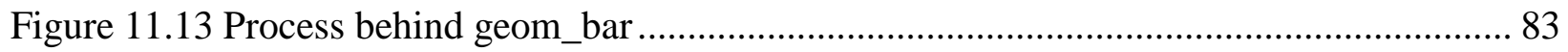

Figure 11.14 Bar chart of diamonds by cut created with stat_count ...................................... 84

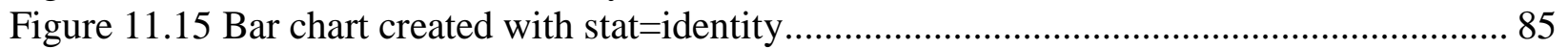

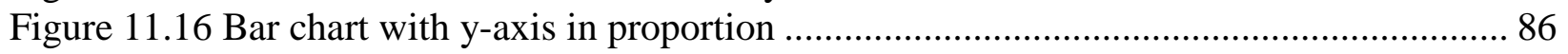

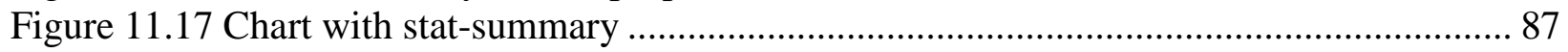

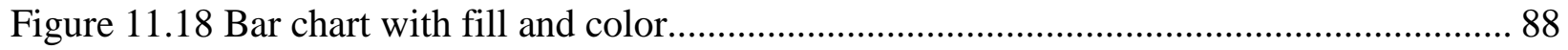

Figure 11.19 Stacked bar chart of diamonds by cut and clarity.......................................... 89

Figure 11.20 Bar chart with position='identity' .............................................................. 90

Figure 11.21 Bar chart with position='fill' ............................................................... 91

Figure 11.22 Bar chart with position='identity' .......................................................... 92

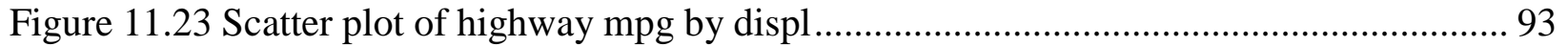

Figure 11.24 Scatter plot of highway mpg by displ, with position='jitter'................................ 94

Figure 11.25 Bar chart with flipped $\mathrm{x}$ and $\mathrm{y}$ axis......................................................... 95

Figure 11.26 Map of New Zealand with ggplot2 ............................................................ 96

Figure 11.27 Coxcomb chart with the flipped and polar coordinate system ........................... 97

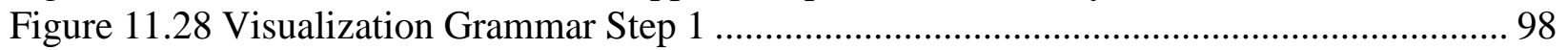

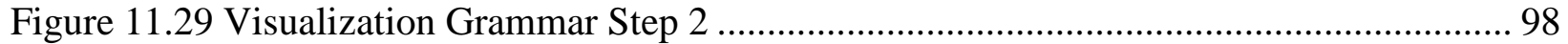

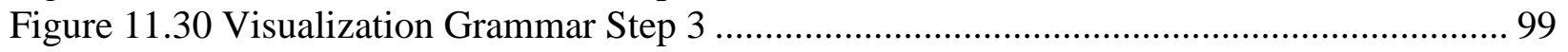

Figure 12.1 Distributions of model intercepts and slopes and their relationship in a scatter plot 


\section{EXECUTIVE SUMMARY}

With almost every aspect of transportation research and practice driven to utilize complex computer software and innovative data sources (University Transportation Research Center, 2014), researchers and professionals increasingly face the computing challenges that have plagued other science and engineering disciplines. Most students of science, engineering, and planning are never taught to build, use, validate, and share software well (Merali, 2010). To help students and professionals in transportation research cope with these challenges, this project will apply lessons from similar programs in other disciplines and aims to equip them with proper scientific computing skills.

The direct outcomes of this project are a course plan and materials for training transportation students and professionals in basic data science. The course materials are open source under the Creative Commons (CC) license and publicly available online on GitHub at https://cities.github.com/datascience. Any school or instructor can replicate the course. The course would also be helpful to transportation practitioners who may use it for self-instruction.

The intangible outcomes of this project will be groups of transportation students who are better equipped for transportation research in the age of computing, and who can spend less time wrestling with software and more time doing useful research.

The project identifies and absorbs the best practices in data science in the academic and professional literature (for example, G. Wilson et al., 2014) as well as successful courses and workshops for similar purposes, such as the Software Carpentry lessons, and develops the following course topics:

- $\quad$ Best practices in data science

- $\quad$ Coding and scripting basics

- $\quad$ Version control using Git

- The import-tidy-transform-visualize-model-communicate workflow

- Communication and reproducible research

- How to find help

The course was first offered as a Transportation Research and Education Consortium (TREC) summer course in 2017. Future offerings of the course are being planned. 


\subsection{SYLLABUS}

Did you ever feel you are "drinking from a hose” with the amount of data you are attempting to analyze? Have you been frustrated with the tedious steps in your data processing and analysis process and thinking, “There's gotta be a better way to do things”? Are you curious what the buzz of data science is about? If any of your answers are yes, then this course is for you.

Although computing is now integral to every aspect of science and engineering, transportation research included, most students of science, engineering, and planning are never taught how to build, use, validate, and share software well. As a result, many spend hours or days doing things badly that could be done well in just a few minutes and in a repeatable and self-documented way. The goal of this course is to empower students to spend less time wrestling with software and more time doing useful research/work.

This course builds on successful data science training programs, such as the Software Carpentry (http://www.software-carpentry.org/) and Data Carpentry, and recent developments in related software and research. It exposes transportation students and professional to the best practices in data science and scientific computing through lectures, discussion, and hands-on lab sessions, and aims to help them tackle the challenge of "drinking from a hose" when dealing with an overwhelming amount of data.

\subsection{FORMAT, SOFTWARE AND HARDWARE}

Classes will all be hands-on sessions with lectures, discussions, and labs. A major component of the class is the class project, in which students go through actual data processing, analysis and reporting while learning the best practices of data science.

This course will use the free statistical software R, with RStudio (https://www.rstudio.com/) as the main interface. The lecture and lab instructions will use R. It is possible (and encouraged) for existing Python users (and potentially users of other software, such as Stata, Matlab, etc.) to keep using the software they already know well. Students are encouraged to bring their own laptops. The instructor and TA will help the students set laptops up to run all examples and exercises in lectures and labs, and to re-run them later for review and their own project.

\subsection{PREREQUISITE}

Basic knowledge and experience of working with quantitative data; experiences and skills in (or keenness to learn) a programming language (e.g., Python); and/or data processing and statistical software (e.g., R, Matlab, Stata).

\subsection{TEXTBOOK AND READINGS}

The course will use the following textbook:

- Wickham, H., Grolemund, G., 2017. R for Data Science: Import, Tidy, Transform, Visualize, and Model Data, 1 edition. ed. O'Reilly Media. (R4DS)

An electronic version is available on Hadley Wickham's website. 
For Python users, Wes McKinney's book is recommended:

- McKinney, W., 2012. Python for Data Analysis: Data Wrangling with Pandas, NumPy, and IPython, 1 edition. ed. O'Reilly Media.

Journal articles and online resources are used as supplements to the textbook.

\subsection{TOPICS}

Topics are tentative and subject to change according to students' needs, but will be centered on these topics:

- $\quad$ Part I:

1. Overview and Introduction

2. R Coding Basics

3. Version Control with Git

4. Create R Package

5. Producing Reports with R Markdown

- $\quad$ Part II:

1. Data Importing

2. Tidy Data

3. Data Manipulation with dplyr

4. Data Visualization with ggplot2

5. Split-Apply-Combine Pattern in Data Modeling

6. Where To Go From Here

\subsection{LICENSE}

The course materials are made available under the Creative Commons Attribution license.

\subsection{ACKNOWLEDGEMENTS}

This course is developed with financial support from the National Institute of Transportation and Communities for project \#854.

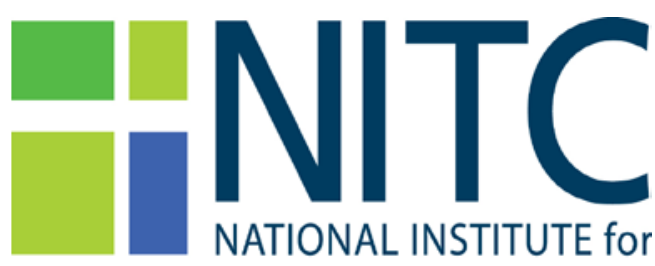

TRANSPORTATION and COMMUNITIES 
I am grateful to the participants of the 2017 summer course for their valuable feedback. The support of TREC staff, in particular, Lisa Patterson and Eva-Maria Muecke, is greatly appreciated. Jamaal Green and Dillon Mahmoudi, both graduate students at Portland State University, have given me valuable feedback and assistance in the process of developing the course.

Parts of the course materials have been adapted from the following sources:

- $\quad$ R for Data Science by Hadley Wickham

- $\quad$ Software Carpentry workshop lessons

- $\quad$ UBC Stat 545 by Professor Jenny Bryan at UBC

- $\quad$ NEU 5110 Introduction to Data Science by Professor Jan Vitek

The writeup and website are powered by the bookdown package and GitHub. 


\subsection{OVERVIEW AND INTRODUCTION}

\subsection{PART I: DATA SCIENCE BEST PRACTICES}

1. Overview and Introduction

2. R Coding Basics

3. Version Control with Git

4. Create R Package

5. Producing Reports with R Markdown

\subsection{SET UP YOUR COMPUTER}

\subsubsection{Installation}

- Install R 3.4.1 from https://ftp.osuosl.org/pub/cran/. (Requires administrator privileges)

- Install RStudio Desktop free version from https://www.rstudio.com/products/rstudio/download/

- Git: Follow these steps to install Git for Windows (or for the specific operating system on your laptop). (Requires administrator privileges)

\subsubsection{Installation Verification}

1. Launch RStudio and you should see a program window like this:

2. Click the File menu, select New Project..., then Version Control and Git;

3. Copy \& paste this URL: https://github.com/cities/datascience2017.git into the Repository URL textbox;

4. Click Create Project.

If you see a popup box that says "Clone Repository" with a progress bar and then RStudio refreshes, then your installation is working.

\section{$2.3 \quad$ WHY R}

- $\quad$ Free, as in beer and speech

- $\quad$ Large and growing community, with more than 13,000 packages and growing

- $\quad$ Powerful and flexible

- $\quad$ An (incomplete) list of models implemented as R packages

- Graphics Gallery

- $\quad$ Interactive Web Apps: Interactive Plots, Dashboard, Widget ...

\subsection{INTRODUCTION TO R AND RSTUDIO}

1. Introduction to R and RStudio

2. Seeking help 


\subsubsection{Project Organization in RStudio}

R experts keep all the files associated with a project together — input data, R scripts, analytical results, and figures. This is such a wise and common practice that RStudio has built-in support for this via projects. For example, a project can be an R package under development, or code, data, and documents for a research project, or a book project (for example, Hadley use an RStudio project for the R for Data Science book at https://github.com/hadley/r4ds).

An RStudio project must contain a file with extension Rproj and can contain any files within the directory where the project file lives or its subdirectories. Typical types of files in an RStudio project include R Script - R Markdown, R notebook - R Presentation - Shiny Web App - Text file, $\mathrm{C}++$, HTML, ...

Hadley recommends the following for RStudio projects workflow:

- $\quad$ Create an RStudio project for each data analysis project.

- $\quad$ Keep data files there and load them into R with data import.

- $\quad$ Keep scripts there; edit them, run them in bits or as a whole.

- $\quad$ Save your outputs (plots and cleaned data) there.

- $\quad$ Only ever use relative paths (relative to the root path of the project), not absolute paths.

Everything you need is in one place and cleanly separated from all the other projects that you are working on.

I recommend organizing files in subdirectories by types, typically with at least these items: project_name.Rproj, RStudio project file - README.md, a description file of the project - code, for R scripts - data, for data files - docs, for document/report files, such as R Markdown files

\subsection{EXERCISE}

Create an RStudio project for this class on your laptop, set up the directory structure as recommended above, download the bike counts data file, and save them in the data directory of your RStudio project.

\subsection{CLASS PROJECT}

For the class project, you are expected to create an R package with the following requirements and commit it to GitHub:

1. Contains at least one self-contained function;

2. Completed with necessary documentation;

3. Passes R package check and test(s); and

4. [Advanced] includes a vignette that demonstrates the usage of the package.

You can take and/or re-organize code from your current work or start from scratch. Take the feasibility of completing it in a week into consideration when selecting project ideas. 
If you don't have a feasible project idea at the moment, consider writing an $\mathrm{R}$ package that reads and visualizes the bike counts on Hawthorne Bridge and Tilikum Crossing. Daily traffic counts data for these two bridges can be found here. At the minimum, your package should be able to:

- $\quad$ Read the data in the excel files;

- $\quad$ Process (tidy) data as necessary;

- Visualize bike counts on either or both bridges based on the data frame passed in;

- $\quad$ Plot daily bike counts for any specified period;

- $\quad$ [Advanced] Plot daily, weekly, or monthly bike counts based on a frequency argument;

- Pull weather data for Portland and study (visualize and/or model) the effect of weather on bike counts; and

- [Advanced] Study (visualize and/or model) the effect of Tilikum Crossing (opened in September 2015) on bike counts on Hawthorne Bridge.

\subsection{LEARNING MORE}

The above will get your basic setup ready but here are some links if you are interested in reading a bit further.

- $\quad$ RStudio Cheat Sheet

- https://www.rstudio.com/wp-content/uploads/2016/01/rstudio-IDE-cheatsheet.pdf

- $\quad$ RStudio's leads for getting help with R

- $\quad$ https://support.rstudio.com/hc/en-us/articles/200717153-Getting-Help-with-R

- R FAQ:

- http://cran.r-project.org/doc/FAQ/R-FAQ.html

- $\quad$ R Installation and Administration

- http://cran.r-project.org/doc/manuals/R-admin.html

- $\quad$ More about add-on packages in the R Installation and Administration Manual

- https://cran.r-project.org/doc/manuals/R-admin.htm|\#Add_002don-packages 


\subsection{R CODING BASICS}

\subsection{READINGS}

1. Wilson, G., Aruliah, D. A., Brown, C. T., Hong, N. P. C., Davis, M., Guy, R. T., ... Wilson, P. (2014). Best Practices for Scientific Computing. PLOS Biology, 12(1), e1001745. https://doi.org/10.1371/journal.pbio.1001745

2. R for Reproducible Scientific Analysis by Software Carpentry

\subsection{BEST PRACTICES FOR DATA SCIENCE}

These are the best practices for data science recommended by G. Wilson et al. (2014):

1. Write programs for people, not computers

i. a program should not require its readers to hold more than a handful of facts in memory at once

ii. make names consistent, distinctive, and meaningful

iii. make code style and formatting consistent

2. Let the computer do the work

i. make the computer repeat tasks

ii. save recent commands in a file for re-use

iii. use a build tool to automate workflows

3. Make incremental changes

i. $\quad$ work in small steps with frequent feedback and course correction

ii. use a version control system

iii. put everything that has been created manually in version control

4. Don't repeat yourself (or others)

i. every piece of data must have a single authoritative representation in the system

ii. modularize code rather than copying and pasting

iii. re-use code instead of rewriting it

5. Plan for mistakes

i. add assertions to programs to check their operation

ii. use an off-the-shelf unit testing library

iii. turn bugs into test cases

iv. use a symbolic debugger

6. Optimize software only after it works correctly

i. use a profiler to identify bottlenecks

ii. write code in the highest-level language possible

7. Document design and purpose, not mechanics

i. document interfaces and reasons, not implementations

ii. refactor code in preference to explaining how it works

iii. embed the documentation for a piece of software in that software

8. Collaborate

i. use pre-merge code reviews

ii. use pair programming when bringing someone new up to speed and when tackling particularly tricky problems

iii. use an issue tracking tool 
In small groups, each picks (or is assigned) a practice and discusses:

1. What is the current practice? Why it is not a good practice and why is the best practice better?

2. How can we move the current practice to the best practice?

\subsection{R CODING BASICS}

This section assumes that students know little about R and gets them up to speed with the basics:

1. Data Structures

- $\quad$ How can I read data in R?

- What are the basic data types in R?

- How do I represent categorical information in R?

2. Exploring Data Frames

- How can I manipulate a data frame?

3. Subsetting Data

- $\quad$ How can I work with subsets of data in R?

4. Control Flow

- How can I work with subsets of data in R?

5. Visualization with ggplot2

- How can I create publication-quality graphics in R?

6. Vectorization

- How can I operate on all the elements of a vector at once?

7. Functions Explained

- $\quad$ How can I write a new function in R?

8. Writing Good Software

- How can I write software that other people can use?

\subsection{ADVANCED TOPICS}

\subsubsection{Code Style Guide}

In programming, as in writing, it is generally a good idea to stick to a consistent coding style. There are two style guides that you can adopt or customize to create your own:

- $\quad$ Google's R style guide

- Hadley Wickham's code style guide

\subsubsection{R Command-Line Program}

RStudio is good for writing and testing your R code, but for work that needs repetition or takes a long time to finish, it may be easier to run your program/script in command line instead. Before we start, open the RStudio project you created following the RStudio project organization recommendations in the Overview section (assuming you downloaded and saved the bike counts data to the data directory of the project).

We can create an R script (from the File/New File/R Script menu of RStudio) that loads the bike counts for the Hawthorne Bridge: 
library(tidyverse)

input_file <- "data/Hawthorne Bridge daily bike counts 2012-2016 082117.xlsx"

bridge_name $<-$ "Hawthorne"

bikecounts $<$-read_excel(input_file)

names(bikecounts) <- c("date", "westbound", "eastbound", "total")

bikecounts\$bridge $<$ - bridge_name

head(bikecounts)

Choose a file name, for example, load_data.R, and save the script in the code directory of your RStudio project (create a code directory first if you haven't yet).

Now we can run the script in a command line shell (you can open one in RStudio's

Tools/Shell... menu):

Rscript code/load_data.R

Notice that the script may not print out outputs on the screen when called in the command line unless you explicitly call the print function.

But what if we have many files for which we would like to repeatedly show the basic information (rows, data types, etc.)? We can refactor our script to accept the file name and bridge name from command line arguments so that the script can work with any acceptable files.

In an R script, you can use the commandArgs function to get the command line arguments:

args $<$ - commandArgs()

print(args)

So, in our case, our script should take input_file and bridge_name from the command line arguments, we can get the value of the arguments with:

args $<-$ commandArgs( $)$

input_file $<-$ args[1]

bridge_name $<-$ args[2]

Replace the two lines in load_data.R starting with input_file and bridge_name with these three lines.

Now our script can be invoked in the command line with:

Rscript code/load_data.R "data/Hawthorne Bridge daily bike counts 2012-2016 082117.xlsx" \ Hawthorne

(The quotation marks are needed for the file name when there are spaces in the name and "।" breaks a command into two lines.) 


\subsubsection{Debugging with RStudio}

This section is adapted from Visual Debugging with RStudio.

1. Download foo.R from

https://raw.githubusercontent.com/cities/datascience2017/master/code/foo.R and save it to the code directory of your project folder;

2. Open foo.R and source it;

3. In the RStudio Console pane of type foo("-1") and then enter.

Why does the foo function claim "-1 is larger than 0"? Let's debug the foo function and find out.

\subsection{EXERCISE}

1. Write a function that takes the name of a bike counts data file as input and return a data frame;

- $\quad$ use the readxl package to read data in excel files

2. Create an R script that utilizes your function to read in data in the Tilikum and Hawthorne bike count files;

3. Do quick summaries of the data for each bridge:

- How many days of data are there for each bridge?

- What are the average daily bike counts for each bridge? Minimum? Maximum?

- What are the average weekly, monthly, and annual bike counts for each bridge?

4. [Advanced] Write a function that calculates average daily, weekly, or monthly bike counts for each bridge based on a frequency argument.

\subsection{LEARNING MORE}

- Introduction to R on Data Camp: A self-instruction course covering R basics.

- $\quad$ Try R by Code School 


\subsection{VERSION CONTROL WITH GIT}

\subsection{READINGS}

- Happy Git and GitHub for the useR

\subsection{INSTALL GIT}

\subsubsection{For Windows}

1. Download the Git for Windows installer.

2. Run the installer and follow the steps below:

1. Click "Next".

2. Click "Next".

3. Keep "Use Git from the Windows Command Prompt" selected and click "Next". If you forgot to do this, rerun the installer and select the appropriate option.

4. Click "Next".

5. Keep "Checkout Windows-style, commit Unix-style line endings" selected and click "Next".

6. Keep "Use Windows' default console window" selected and click "Next".

7. Click on "Install".

8. Click "Finish".

3. If your "HOME" environment variable is not set (or you don't know what this is):

1. Open command prompt (Open Start Menu then type cmd and press [Enter])

2. Type the following line into the command prompt window exactly as shown: setx HOME "\%USERPROFILE\%"

3. Press [Enter], you should see SUCCESS: Specified value was saved.

4. Quit command prompt by typing exit then pressing [Enter].

\subsubsection{For Mac}

Install git-osx-installer.

\subsubsection{For Linux}

install git from your Linux distro's package manager.

\subsection{LESSON}

Adapted from the Software Carpentry lesson Version Control with Git and Professor Jenny Bryan's Happy Git with R.

- $\quad$ A quick overview of Version Control;

- $\quad$ Register a Github account at https://github.com;

- $\quad$ Use your GitHub username and email to config Git installation on your own computer.

Open a Shell/Command line window from RStudio menu Tools/Shell..., and modify and run the following commands (of course, replace my name and email with your own): 
git config --global user.name "Liming Wang"

git config --global user.email "lmwang@gmail.com"

git config --global color.ui "auto"

- $\quad$ Cache credentials

- Collaborating

- Conflicts

- Review of common git commands

- $\quad$ Using Git fro RStudio

\subsection{EXERCISE}

1. Set up your Git installation for use with GitHub;

2. Turn the RStudio project you have been working on into a Git repository;

3. Commit it to GitHub by following these steps.

\subsection{LEARNING MORE}

1. Happy Git with $\mathrm{R}$

2. Software Carpentry's Version Control with Git

3. $\quad$ Try Git

4. Pro Git, a book by Scott Chacon and Ben Straub 


\subsection{CREATE R PACKAGE}

\subsection{READINGS}

- $\quad$ R packages Getting Started

\subsection{LESSON}

1. Developing R Packages with RStudio

2. Building, Testing, and Distributing Packages

3. Writing Package Documentation

4. Writing Documents for R Package

\subsection{R PACKAGE CHECKLIST}

0. Check out (clone) the package repository as an RStudio project or create an R package project from RStudio menu File/New Project...;

1. Test that the package builds and loads without issue;

2. Check the package with devtools::check(), which also runs tests included in the package; verify there are no errors or warnings;

3. Does the package include tests? Do the tests run successfully?

4. Verify that the package includes proper documents as roxygen comments in the R code files;

5. Does the package include vignettes?

6. Install the package and load it with library;

7. See what functions are provided by the package with ls("package:<package name>") after loading it with library; and

8. Study the vignettes and help documents to learn how to use it.

\subsection{EXERCISE}

Reorganize the function(s) you developed in the R Coding Basics section into an R package:

- $\quad$ Use roxgen comments to specify the dependencies of your function(s);

- $\quad$ Use roxgen comments to document your function(s);

- $\quad$ [Advanced] Create tests for your functions;

- Make sure your package builds and loads properly;

- $\quad$ Go through the R package checklist; and

- $\quad$ Commit your package as a GitHub repository.

\subsection{LEARNING MORE}

1. R Package by Hadley Wickham

2. Making Your First R Package by Fong Chun Chan

3. A Quickstart Guide for Building Your First R Package 


\subsection{R MARKDOWN}

R Markdown provides an authoring framework for data science. You can use a single R Markdown file to both

- $\quad$ save and execute code and

- $\quad$ generate high-quality reports that can be shared with an audience.

R Markdown documents are fully reproducible and support dozens of static and dynamic output formats.

You write R Markdown documents in a simple plain text-like format, which allows you to format text using intuitive notations so that you can focus on the content you're writing. But you still get a well-formatted document out. In fact, you can turn your plain text (and R code and results) into an HTML file or, if you have an installation of LaTeX and Pandoc on your machine, a pdf, or even a Word document (if you must!).

\subsection{READINGS}

1. $\quad$ R4DS Chapter 27, Chapter 30, Chapter 29

2. R Markdown gallery

\subsection{LESSONS}

To get started, install the knitr package.

install.packages("knitr")

When you click on File -> New File, there is an option for "R Markdown...". Choose this and accept the default options in the dialog box that follows (but note that you can also create presentations this way). Save the file and click on the "Knit HTML" button at the top of the script. Compare the output to the source.

\subsubsection{Formatting Text in Markdown}

Visit http://rmarkdown.rstudio.com/authoring_basics.html and briefly check out some of the formatting options.

In the example document add

- Headers using \#

- $\quad$ Emphasis using asterisks: *italics* and **bold**

- $\quad$ Lists using * and numbered lists using 1., 2., etc.

Markdown also supports LaTeX equation editing. We can display pretty equations by enclosing them in $\$$, e.g., $\$ \backslash$ alpha $=\backslash \operatorname{dfrac}\{1\}\left\{(1-\backslash \text { beta })^{\wedge} 2\right\} \$$ renders as: $\alpha=\frac{1}{(1-\beta)^{2}}$. 
The top of the source (.Rmd) file has some header material in YAML format (enclosed by triple dashes). Some of this gets displayed in the output header; some provides formatting information to the conversion engine.

To distinguish R code from text, RMarkdown uses three back-ticks followed by $\{r\}$ to distinguish a "code chunk". In RStudio, the keyboard shortcut to create a code chunk is command-option-i or control-alt-i.

A code chunk will set off the code and its results in the output document, but you can also print the results of code within a text block by enclosing code like so: `r code-here`.

\subsubsection{Use knitr to Produce a Report}

1. Open a new.Rmd script and save it as inflammation_report.Rmd.

2. Copy code from earlier into code chunks to read the inflammation data and plot average inflammation.

3. Add a few notes describing what the code does and what the main findings are. Include an in-line calculation of the median inflammation level.

4. Knit the document and view the HTML result.

\subsection{EXERCISE}

Follow Test drive R Markdown by Professor Bryan to create a report (in HTML format) that includes:

- A brief introduction of the bike counts data (where were the data collected, the period, etc.);

- $\quad$ Load the bike counts data in the R code chunks of your R markdown file;

- $\quad$ Embed summary statistics and visualization of the bike counts data in your report;

- [Advanced] Create a vignette for the R package you created for Create R Package that demonstrates the usage of the package.

\subsection{LEARNING MORE}

1. R Markdown from RStudio

2. R Markdown Cheatsheet

3. Software Carpentry R Markdown Lesson

4. Test Drive R Markdown by Professor Bryan

5. Writing Vignettes with R Markdown 


\subsection{PART II: OVERVIEW AND INTRODUCTION}

Data science is a discipline that combines computing with statistics. A data analysis problem is solved in a series of data-centric steps: data acquisition and representation (Import), data cleaning (Tidy), an iterative sequence of data transformation (Transform), data modeling (Model) and data visualization (Visualize). The end result of the process is to communicate insights obtained from the data (Communicate). Part II of this class will take you through all the steps in the process and will teach you how to approach such problems in a systematic manner. You will learn how to design data analysis pipelines as well as how to implement data analysis pipelines in R. The class will also emphasize how elegant code leads to reproducible science.

\subsection{PART II: TIDYVERSE WORKFLOW}

1. Data Importing

2. Tidy Data

3. Data Manipulation with dplyr

4. Data Visualization with ggplot2

5. Split-Apply-Combine Pattern in Data Modeling

6. Where To Go From Here

\subsection{READINGS}

1. R4DS Chapter 1

\subsection{THE TIDYVERSE WORKFLOW}

First, you must import your data into R. This typically means that you take data stored in a file, database or web API, and load it into a data frame in R. If you can't get your data into R, you can’t do data science on it!

Once you've imported your data, it is a good idea to tidy it. Tidying your data means storing thhhem in a consistent form that matches the semantics of the dataset with the way they are stored. In brief, when your data are tidy, each column is a variable, and each row is an observation. Tidy data are important, because the consistent structure lets you focus your efforts on questions about the data, not fighting to get the data into the right form for different functions.

Once you have tidy data, a common first step is to transform them. Transformation includes narrowing in on observations of interest (like all people in one city, or all data from the last year), creating new variables that are functions of existing variables (like computing velocity from speed and time), and calculating a set of summary statistics (like counts or means).

Together, tidying and transforming are called wrangling, because getting your data in a form that's natural to work with often feels like a fight! 
Once you have tidy data with the variables you need, there are two main engines of knowledge generation: visualization and modeling. These have complementary strengths and weaknesses so any real analysis will iterate between them many times.

Visualization is a fundamentally human activity. A good visualization will show you things that you did not expect, or raise new questions about the data. A good visualization might also hint that you're asking the wrong question, or you need to collect different data. Visualizations can surprise you, but don't scale particularly well because they require a human to interpret them.

Models are complementary tools for visualization. Once you have made your questions sufficiently precise, you can use a model to answer them. Models are a fundamentally mathematical or computational tool, so they generally scale well. Even when they don't, it's usually cheaper to buy more computers than it is to buy more brains! But every model makes assumptions, and by its very nature a model cannot question its own assumptions. That means a model cannot fundamentally surprise you.

The last step of data science is communication, an absolutely critical part of any data analysis project. It doesn't matter h

ow well your models and visualization have led you to understand the data unless you can also communicate your results to others.

Surrounding all these tools is programming. Programming is a cross-cutting tool that you use in every part of the project. You don't need to be an expert programmer to be a data scientist, but learning more about programming pays off because becoming a better programmer allows you to automate common tasks and solve new problems with greater ease.

You'll use these tools in every data science project, but for most projects they're not enough. There's a rough $80-20$ rule at play; you can tackle about $80 \%$ of every project using the tools that you'll learn in this book, but you'll need other tools to tackle the remaining 20\%. Throughout this book, we'll point you to resources where you can learn more.

\subsection{THE TIDYVERSE PACKAGES}

- Website: http://www.tidyverse.org/packages/

- Install with install.packages("tidyverse")

\subsection{PIPE OPERATOR}

$\%>\%$ pipes an object forward into a function or call expression

- $\quad$ Basic piping

- $\quad \mathrm{x} \%>\% \mathrm{f}$ is equivalent to $\mathrm{f}(\mathrm{x})$

- $\quad x \%>\% \mathrm{f}(\mathrm{y})$ is equivalent to $\mathrm{f}(\mathrm{x}, \mathrm{y})$

- $\quad x \%>\%$ f \% $>$ g \% $>\%$ h is equivalent to $h(g(f(x)))$

- $\quad$ Using $\%>\%$ with unary function calls

require(tidyverse)

iris $\%>\%$ head 
\#\# Sepal.Length Sepal.Width Petal.Length Petal.Width Species

$\begin{array}{lllll}\text { \#\# 1 } & 5.1 & 3.5 & 1.4 & 0.2 \text { setosa } \\ \text { \#\# 2 } & 4.9 & 3.0 & 1.4 & 0.2 \text { setosa } \\ \text { \#\# 3 } & 4.7 & 3.2 & 1.3 & 0.2 \text { setosa } \\ \text { \#\# 4 } & 4.6 & 3.1 & 1.5 & 0.2 \text { setosa } \\ \text { \#\# 5 } & 5.0 & 3.6 & 1.4 & 0.2 \text { setosa } \\ \text { \#\# 6 } & 5.4 & 3.9 & 1.7 & 0.4 \text { setosa }\end{array}$

iris $\%>\%$ tail

\#\# Sepal.Length Sepal.Width Petal.Length Petal.Width Species

$\begin{array}{lllll}\text { \#\# } 145 & 6.7 & 3.3 & 5.7 & 2.5 \text { virginica }\end{array}$

$\begin{array}{lllll}\text { \#\# } 146 & 6.7 & 3.0 & 5.2 & 2.3 \text { virginica }\end{array}$

$\begin{array}{lllll}\text { \#\# } 147 & 6.3 & 2.5 & 5.0 & 1.9 \text { virginica }\end{array}$

$\begin{array}{lllll}\text { \#\# } 148 & 6.5 & 3.0 & 5.2 & 2.0 \text { virginica }\end{array}$

$\begin{array}{lllll}\text { \#\# } 149 & 6.2 & 3.4 & 5.4 & 2.3 \text { virginica }\end{array}$

$\begin{array}{lllll}\text { \#\# } 150 & 5.9 & 3.0 & 5.1 & 1.8 \text { virginica }\end{array}$

- $\quad$ Placing lhs as the first argument in rhs call

\#\# What are the outputs of these two lines?

$3 \%>\%{ }^{\prime}-(4)$

iris $\%>\% \operatorname{head}(5)$

iris $\%>\%$ tail(5)

- $\quad$ Placing lhs elsewhere in rhs call

\#\# What are the outputs of these

$3 \%>\%{ }^{\prime}-(4,$.

4 \% > \% c("A", "B", "C", "D", "E")[.]

More information available at http://magrittr.tidyverse.org/

\subsubsection{RStudio Keyboard Shortcut for $\%>\%$}

- $\quad$ ctrl-Shift-M (Windows)

- $\quad$ Command-Shift-M (Mac)

\subsection{CLASS PROJECT}

For the class project for Part II, you are expected to follow the tidyverse workflow and use the tidyverse suite of packages to conduct an analytic project using data of your choice. Your project should involve these steps:

1. Data importing and tidying

2. Data transformation

3. Data visualization

4. [Optional] Data modeling

5. [Optional] Reproducible reporting 
You can use data from your current work or continue a project you have been working on. Take the feasibility of completing it in a week into consideration when selecting project ideas.

If you don't have a feasible project idea at the moment, consider continuing to analyze the bike counts data on Hawthorne Bridge and Tilikum Crossing from Part I. Daily traffic counts data for these two bridges can be found here.

Sample analytic questions are:

- $\quad$ After accounting for seasonal variation, is there a trend in bike traffic across these two bridges?

- How was the bike traffic affected by weather (temperature, precipitation, etc.)?

- Was the bike traffic on the Hawthorne Bridge affected by the opening of Tilikum Crossing?

You can work on any one or all of these questions with visualization and/or modeling after you import, tidy and transform the data when necessary. 


\subsection{DATA IMPORTING}

\subsection{READINGS}

1. R4DS Chapter 10, Chapter 11

\subsection{TIBBLES VS. DATA.FRAME}

\subsubsection{Data Frames are Awesome}

Whenever you have rectangular, spreadsheet-y data, your default data receptacle in $\mathrm{R}$ is a data frame. Do not depart from this without good reason. Data frames are awesome because

- $\quad$ Data frames package related variables neatly together,

- $\quad$ keeping them in sync vis-a-vis row order and

- $\quad$ applying any filtering of observations uniformly.

- Most functions for inference, modeling, and graphing are happy to be passed a data frame via a data = argument. This has been true in base $\mathrm{R}$ for a long time.

- The set of packages known as the tidyverse takes this one step further and explicitly prioritizes the processing of data frames. This includes popular packages like dplyr and ggplot2. In fact, the tidyverse prioritizes a special flavor of data frame, called a "tibble."

Data frames - unlike general arrays or, specifically, matrices in $\mathrm{R}$ - can hold variables of different flavors, such as character data (subject ID or name), quantitative data (white blood cell count), and categorical information (treated vs. untreated). If you use homogenous structures, like matrices, for data analysis, you are likely to make the terrible mistake of spreading a dataset out over multiple, unlinked objects. Why? Because you can't put character data, such as subject name, into the numeric matrix that holds white blood cell count. This fragmentation is a bad idea.

In R Coding Basics we use data.frame in base $\mathrm{R}$ as our main data structure. From now on, we will start to use tibble and other functions in tidyverse as much as possible. This will provide a special type of data frame called a "tibble" that has nice default printing behavior, among other benefits such as speedy performance and better default behavior.

First, install tidyverse packages if you haven't yet; you only need to do this once on your laptop: install.packages("tidyverse")

Then load the tidyverse packages:

library(tidyverse)

There are two main differences in the usage of a tibble vs. a classic data.frame: printing and subsetting. 


\subsubsection{Printing}

Tibbles have a refined print method that shows only the first 10 rows, and all the columns that fit on the screen. This makes it much easier to work with large data. In addition to its name, each column reports its type, a nice feature borrowed from str():

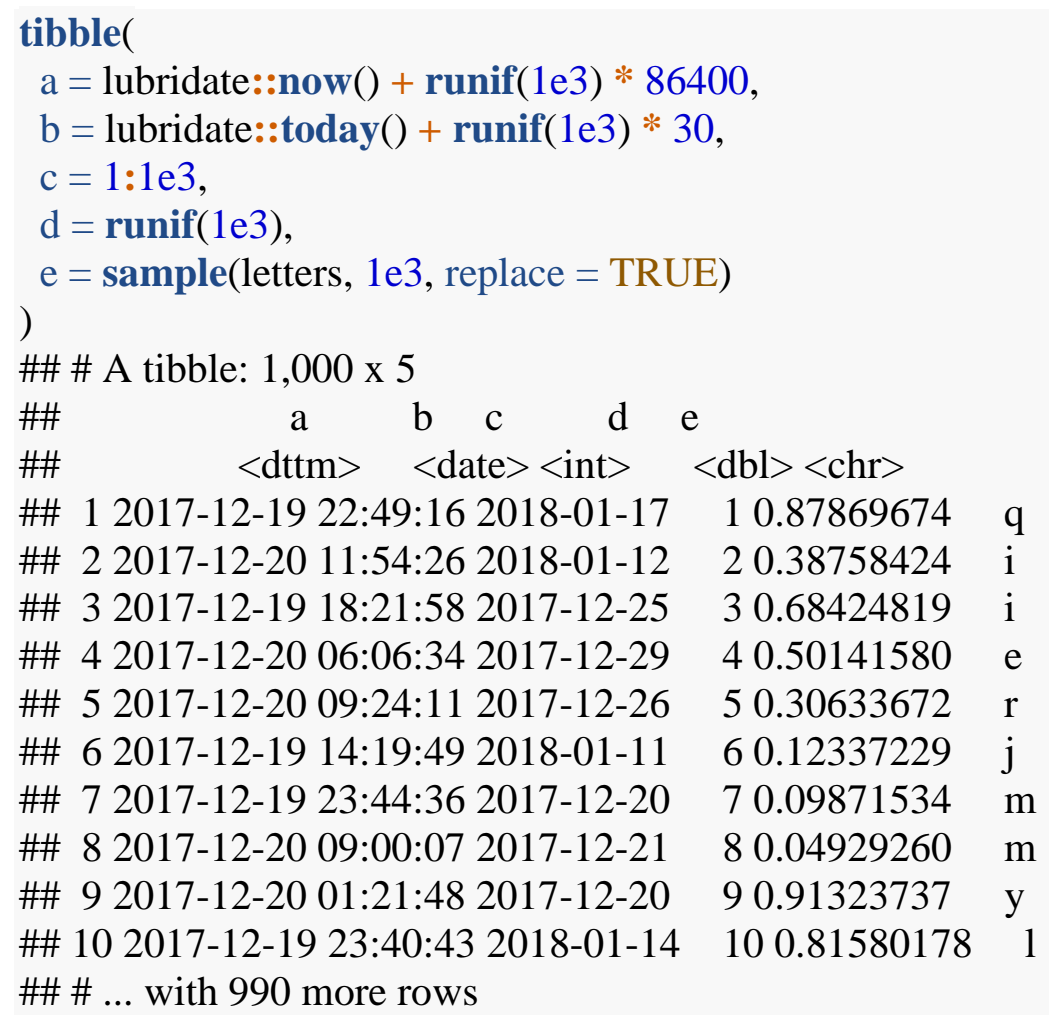

Lubridate is an R package for date and time. You need to install the package if you want to replicate the above code on your own computer.

Tibbles are designed so that you don't accidentally overwhelm your console when you print large data frames. But sometimes you need more output than the default display. There are a few options that can help.

First, you can explicitly print() the data frame and control the number of rows (n) and the width of the display. width = Inf will display all columns:

print (mtcars, $\mathrm{n}=10$, width $=\operatorname{Inf})$

You can also control the default print behavior by setting options:

- options(tibble.print_max $=\mathrm{n}$ ): (Replace $n$ with an actual number, for example, 100) print at most n rows. Use options(dplyr.print_min = Inf) to always show all rows.

- Use options(tibble.width = Inf) to always print all columns, regardless of the width of the screen.

You can see a complete list of options by looking at the package help with ?tibble. 
A final option is to use RStudio's built-in data viewer to get a scrollable view of the complete dataset. This is also often useful at the end of a long chain of manipulations.

View(mtcars)

\subsubsection{Subsetting}

So far all the tools you've learned have worked with complete data frames. If you want to pull out a single variable, you need some new tools, \$ and [[. [[ can extract by name or column position; $\$$ only extracts by name but is a little less typing.

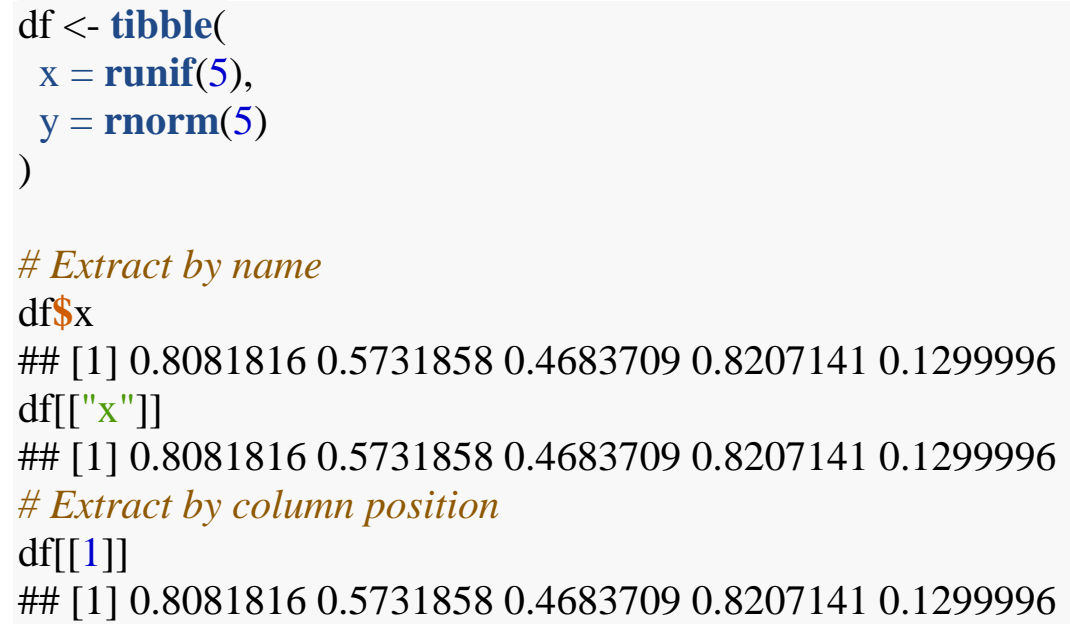

Compared to a data.frame, tibbles are more strict: They never do partial matching, and they will generate a warning if the column you are trying to access does not exist.

\subsubsection{Converting}

Some older functions don't work with tibbles. If you encounter one of these functions, use as.data.frame() to turn a tibble back to a data.frame:

class(as.data.frame(df))

\#\# [1] "data.frame"

Or use as_tibble() to convert a data.frame to tibble:

class(as_tibble(mtcars))

\#\# [1] "tbl_df" "tbl" "data.frame"

The main reason that some older functions don't work with tibble is the [ function. We don't use [ much because dplyr::filter() and dplyr::select() allow you to solve the same problems with clearer code (but you will learn a little about it in vector subsetting). With base R data frames, [ sometimes returns a data frame, and sometimes returns a vector. With tibbles, [ always returns another tibble. 


\subsection{DATA IMPORT}

The most common data in data science are rectangular, spreadsheet-y data that work best to be loaded as an $\mathrm{R}$ data frame, and this section focuses on importing data into $\mathrm{R}$ as data frames. tidyverse provides the readr package that reads comma-separated values (csv), tab-delimited values (tsv), and fixed-width files (fwf) as tibbles. Other R packages provide access to Excel spreadsheets, binary statistical data formats (SPSS, SAS, Stata), and relational databases:

- $\quad$ readr - reads flat files (csv, tsv, fwf) into $\mathrm{R}$

- $\quad$ readxl - reads Excel files (.xls and .xlsx) into R

- $\quad$ heaven - reads SPSS, Stata and SAS files into R

- $\quad$ rstats-db - R interfaces to databases

- RMYSQL

- RSQLite

- RPostgres

For this section, we primarily focus on importing data in flat files into data frames. readr provides these functions:

- read_csv() reads comma-delimited files, and read_csv2() reads semicolon separated files (common in countries where, is used as the decimal).

- $\quad$ read_tsv() reads tab-delimited files, and read_delim() reads in files with any delimiter.

- read_fwf() reads fixed-width files. You can specify fields either by their widths with fwf_widths(), or their position with fwf_positions(). read_table() reads a common variation of fixed width files where columns are separated by white space.

- read_log() reads Apache-style log files. (But also check out webreadr which is built on top of read_log() and provides many more helpful tools.)

These functions all have the similar syntax: Once you've mastered one, you can use the others with ease. For the rest of this chapter, we'll focus on read_csv(). Not only are csv files one of the most common forms of data storage, but once you understand read_csv(), you can easily apply your knowledge to all the other functions in readr.

The first argument to read_csv() is the most important: It's the path to the file to read.

Download heights.csv from this link and save it in the data directory of your RStudio project.

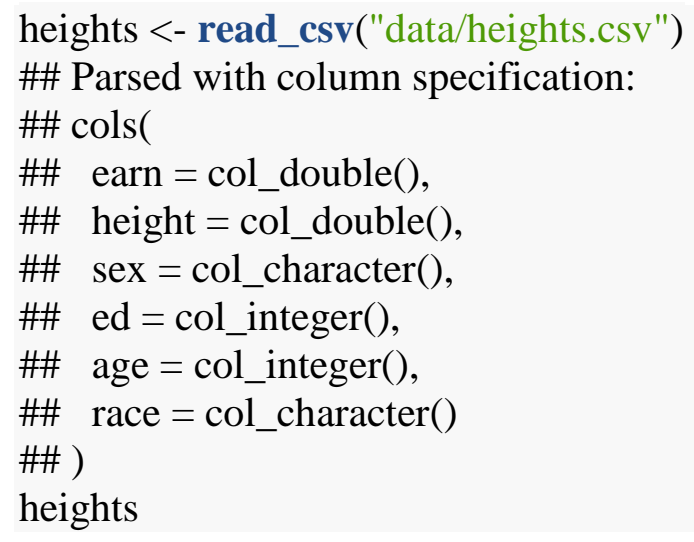




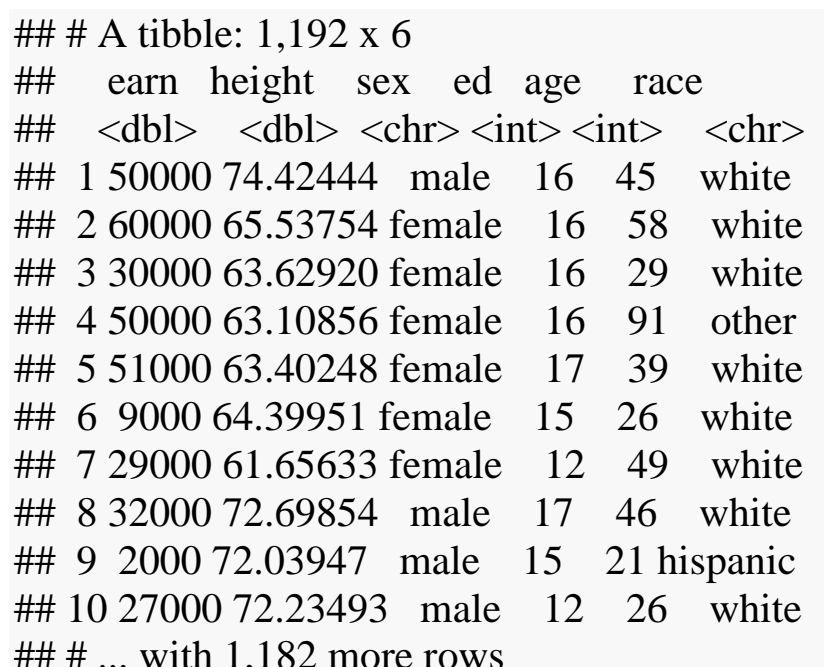

When you run read_csv(), it prints out a column specification that gives the name and type of each column. That's an important part of the readr package and more details of how it works are available in the parsing a file section of R4DS.

read_csv() uses the first line of the data for the column names, which is a very common convention. There are two cases where you might want to tweak this behavior:

1. Sometimes there are a few lines of metadata at the top of the file. You can use skip $=\mathrm{n}$ to skip the first $n$ lines, or use comment = "\#" to drop all lines that start with (e.g.) \#.

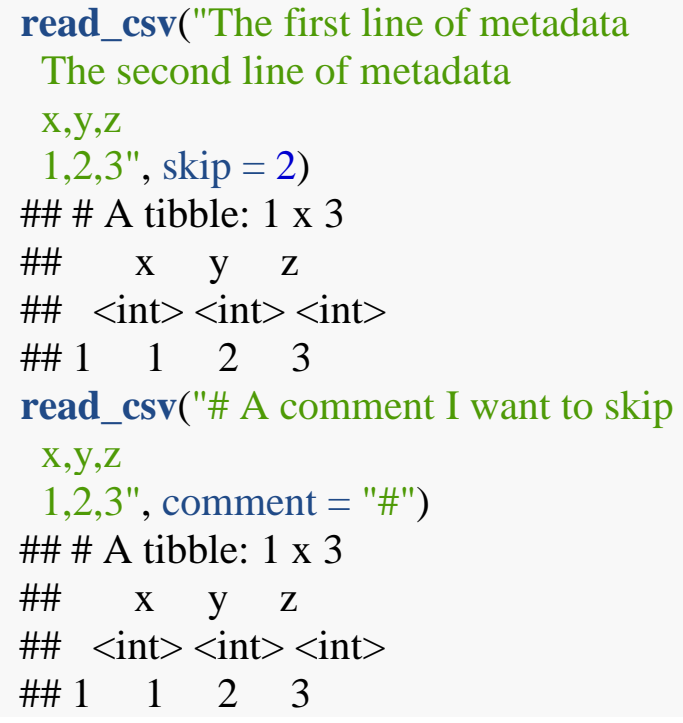

2. The data might not have column names. You can use col_names = FALSE to tell read_csv() not to treat the first row as headings, and instead label them sequentially from X1 to Xn:

read_csv("1,2,3\n4,5,6", col_names = FALSE)

\#\# \# A tibble: 2 x 3

\#\# $\quad$ X1 1 X2 X3 
\#\# $<$ int $><$ int $><$ int $>$

\#\# $1112 \quad 2 \quad 3$

\#\# $2 \quad 4 \quad 5 \quad 6$

("\n" is a convenient shortcut for adding a new line. You'll learn more about it and other types of string escape in string basics.)

Alternatively, you can pass col_names a character vector to use as the column names:

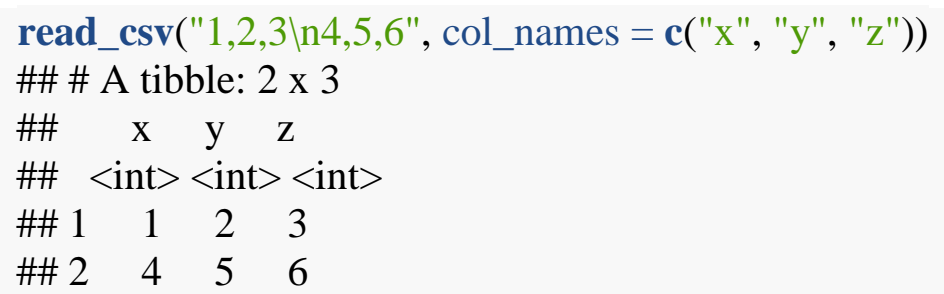

Another option that commonly needs tweaking is na: This specifies the value (or values) that are used to represent missing values in your file:

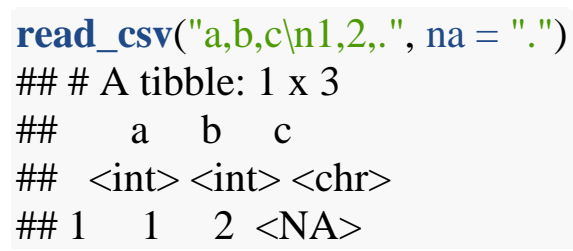

This is all you need to know to read $~ 75 \%$ of CSV files that you'll encounter in practice. You can also easily adapt what you've learned to read tab-separated files with read_tsv() and fixed-width files with read_fwf(). To read more challenging files, you'll need to learn more about how readr parses each column, turning them into $\mathrm{R}$ vectors.

\subsection{EXERCISE}

\section{Link to the README file for the data}

1. What is the difference between a data.frame and tibble? How do you convert between them?

2. Import the bike counts data for Hawthorne and Tilikum in Microsoft Excel format;

3. Import the Portland weather data in csv format;

4. [Challenge] Import the Portland weather data in fixed-width format;

5. For those already familiar with $\mathrm{R}$, create an R script that loads, cleans, and visualizes the bike counts data as well as temperature and precipitation data (using data from Weather Station USC00356750).

For those not yet familiar with $\mathrm{R}$, think about how you would go about doing these tasks with the software you are most comfortable with. 


\subsection{LEARNING MORE}

- $\quad$ R Data Import Tutorial by Data Camp

- Importing Data and Exporting Data by R Programming @ UC.

- $\quad$ Data Import \& Export in R by National Park Services

- $\quad$ Scrape web data with APIs 


\subsection{TIDY DATA}

\subsection{READINGS}

1. R4DS Chapter 12

2. Wickham, Hadley, 2014. Tidy Data, Journal of Statistical Software Vol 59 (2014), Issue 10, 10.18637/jss.v059.i10

\subsection{TIDY DATA}

There are three interrelated rules, which make a dataset tidy:

- $\quad$ Each variable must have its own column.

- $\quad$ Each observation must have its own row.

- $\quad$ Each value must have its own cell.

That interrelationship leads to an even simpler set of practical instructions:

- $\quad$ Put each dataset in a tibble.

- $\quad$ Put each variable in a column.

In this session, you will learn a consistent way to organize your data in $\mathrm{R}$, an organization called tidy data. Getting your data into this format requires some upfront work, but that work pays off in the long term. Once you have tidy data and the tidy tools provided by packages in the tidyverse, you will spend much less time munging data from one representation to another, allowing you to spend more time on the analytic questions at hand. This session will give you a practical introduction to tidy data and the accompanying tools in the tidyr package.

\subsection{LESSON}

table1 - table4 (loaded with the tidyverse package) below shows the same data organized in four different ways. Each dataset shows the same values of four variables country, year, population, and cases, but each dataset organizes the values in a different way:

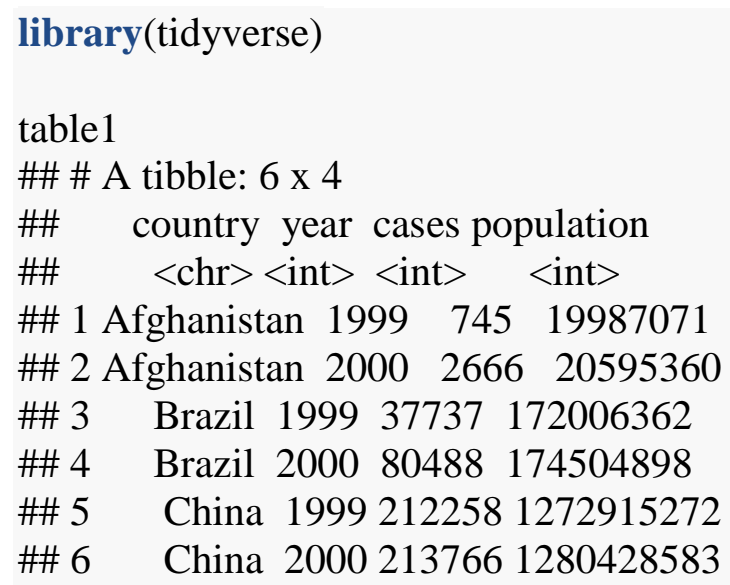


table2

\#\# \# A tibble: 12 x 4

\#\# country year type count

\#\#<chr><int> <chr> <int $>$

\#\# 1 Afghanistan 1999 cases 745

\#\# 2 Afghanistan 1999 population 19987071

\#\# 3 Afghanistan 2000 cases 2666

\#\# 4 Afghanistan 2000 population 20595360

\#\# 5 Brazil 1999 cases 37737

\#\# 6 Brazil 1999 population 172006362

\#\# 7 Brazil 2000 cases 80488

\#\# 8 Brazil 2000 population 174504898

\#\# 9 China 1999 cases 212258

\#\# 10 China 1999 population 1272915272

\#\# 11 China 2000 cases 213766

\#\# 12 China 2000 population 1280428583

table3

\#\# \# A tibble: 6 x 3

\#\# country year rate

\#\# * <chr $><$ int $>\quad<$ chr $>$

\#\# 1 Afghanistan 1999 745/19987071

\#\# 2 Afghanistan $2000 \quad$ 2666/20595360

\#\# 3 Brazil 1999 37737/172006362

\#\# 4 Brazil 2000 80488/174504898

\#\# 5 China 1999 212258/1272915272

\#\# 6 China 2000 213766/1280428583

\# Spread across two tibbles

table4a \# cases

\#\# \# A tibble: 3 x 3

\#\# country '1999`'2000`

\#\# * < chr $><$ int $><$ int $>$

\#\# 1 Afghanistan 7452666

\#\# 2 Brazil 3773780488

\#\# 3 China 212258213766

table4b \# population

\#\# \# A tibble: 3 x 3

\#\# country '1999' '2000`

\#\# * <chr $>\quad<$ int $>\quad<$ int $>$

\#\# 1 Afghanistan 1998707120595360

\#\# 2 Brazil 172006362174504898

\#\# 3 China 12729152721280428583

These are all representations of the same underlying data, but they are not equally easy to use.

One dataset, the tidy dataset, will be much easier to work with inside the tidyverse. 


\subsubsection{Spreading and Gathering}

The principles of tidy data seem so obvious that you might wonder if you'll ever encounter a dataset that isn't tidy. Unfortunately, however, most data that you will encounter will be untidy. There are two main reasons:

1. Most people aren't familiar with the principles of tidy data, and it's hard to derive them yourself unless you spend a lot of time working with data.

2. Data are often organized to facilitate some use other than analysis. For example, data are often organized to make entry as easy as possible.

This means for most real analyses, you'll need to do some tidying. The first step is always to figure out what the variables and observations are. Sometimes this is easy; other times you'll need to consult with the people who originally generated the data. The second step is to resolve one of two common problems:

1. One variable might be spread across multiple columns.

2. One observation might be scattered across multiple rows.

Typically, a dataset will only suffer from one of these problems; it'll only suffer from both if you're really unlucky! To fix these problems, you'll need the two most important functions in tidyr: gather() and spread().

\subsubsection{Gathering}

A common problem is a dataset where some of the column names are not names of variables, but values of a variable. Take table4a: the column names 1999 and 2000 represent values of the year variable, and each row represents two observations, not one.

table4a

\#\# \# A tibble: 3 x 3

\#\# country '1999' '2000`

\#\#* $\quad<$ chr $><$ int $><$ int $>$

\#\# 1 Afghanistan 7452666

\#\# 2 Brazil 3773780488

\#\# 3 China 212258213766

To tidy a dataset like this, we need to gather those columns into a new pair of variables. To describe that operation we need three parameters:

- The set of columns that represent values, not variables. In this example, those are the columns 1999 and 2000.

- The name of the variable whose values form the column names. I call that the key, and here it is year.

- The name of the variable whose values are spread over the cells. I call that value, and here it's the number of cases.

Together those parameters generate the call to gather(): 
table4a $\%>\%$

gather( (1999`, `2000`, key = "year", value = "cases")

\#\# \# A tibble: 6 x 3

\#\# country year cases

\#\# $\quad<$ chr $><$ chr $><$ int $>$

\#\# 1 Afghanistan 1999745

\#\# 2 Brazil 199937737

\#\# 3 China 1999212258

\#\# 4 Afghanistan 20002666

\#\# 5 Brazil 200080488

\#\# 6 China 2000213766

The columns to gather are specified with dplyr::select() style notation. Here there are only two columns, so we list them individually. Note that "1999" and "2000" are non-syntactic names so we have to surround them in backticks. We will learn more about select in Data manipulation with dplyr.

\begin{tabular}{|c|c|c|c|c|c|}
\hline country & year & cases & country & 1999 & 2000 \\
\hline Afghanistan & 1999 & 745 & & & 2666 \\
\hline Afghanistan & 2000 & 2666 & Brazil & 377 & 80488 \\
\hline Brazil & 1999 & 37737 & & & 213766 \\
\hline Brazil & 2000 & 00 & & & \\
\hline China & 1999 & 212258 & & & \\
\hline China & 2000 & 213766 & & & \\
\hline
\end{tabular}

Figure 9.1 Gathering table4 into a tidy form

In the final result the gathered columns are dropped, and we get new key and value columns. Otherwise, the relationships between the original variables are preserved. Visually, this is shown in Figure 9.1. We can use gather() to tidy table4b in a similar fashion. The only difference is the variable stored in the cell values:

table4b \%>\%

gather( '1999`, '2000`, key = "year", value = "population")

\#\# \# A tibble: 6 x 3

\#\# country year population

\#\#<chr $><$ chr $>\quad<$ int $>$

\#\# 1 Afghanistan 199919987071

\#\# 2 Brazil 1999172006362

\#\# 3 China 19991272915272

\#\# 4 Afghanistan 200020595360

\#\# 5 Brazil 2000174504898

\#\# 6 China 20001280428583 
To combine the tidied versions of table4a and table4b into a single tibble, we need to use dplyr::left_join(), which you'll learn about in Working with two datasets.

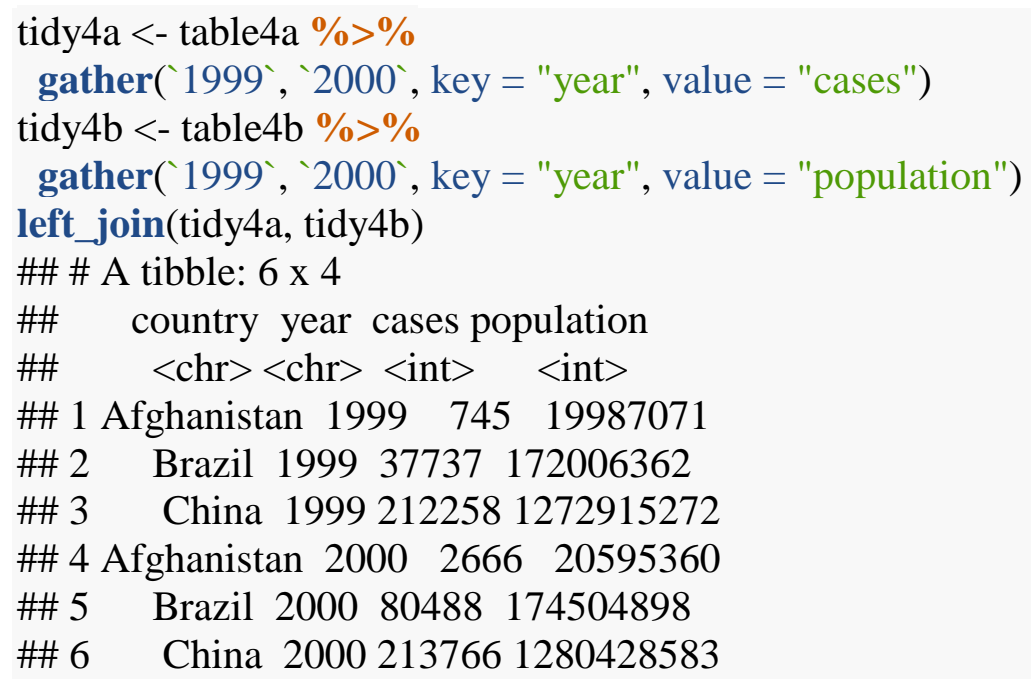

\subsubsection{Spreading}

Spreading is the opposite of gathering. You use it when an observation is scattered across multiple rows. For example, take table2: an observation is a country in a year, but each observation is spread across two rows.

table2

\#\# \# A tibble: 12 x 4

\#\# country year type count

\#\# $\quad<$ chr $><$ int $>\quad<$ chr $>\quad<$ int $>$

\#\# 1 Afghanistan 1999 cases 745

\#\# 2 Afghanistan 1999 population 19987071

\#\# 3 Afghanistan 2000 cases 2666

\#\# 4 Afghanistan 2000 population 20595360

\#\# 5 Brazil 1999 cases 37737

\#\# 6 Brazil 1999 population 172006362

\#\# 7 Brazil 2000 cases 80488

\#\# 8 Brazil 2000 population 174504898

\#\# 9 China 1999 cases 212258

\#\# 10 China 1999 population 1272915272

\#\# 11 China 2000 cases 213766

\#\# 12 China 2000 population 1280428583

To tidy this up, we first analyze the representation in a similar way to gather(). This time, however, we only need two parameters:

- The column that contains variable names, the key column. Here, it's type.

- The column that contains values forms multiple variables, the value column. Here it's count. 
Once we've figured that out, we can use spread(), as shown programmatically below, and visually in Figure 9.2.

spread(table2, key = type, value $=$ count $)$

\#\# \# A tibble: 6 x 4

\#\# country year cases population

\#\# * <chr $><$ int $><$ int $>\quad<$ int $>$

\#\# 1 Afghanistan 199974519987071

\#\# 2 Afghanistan 2000266620595360

\#\# 3 Brazil 199937737172006362

\#\# 4 Brazil 200080488174504898

\#\# 5 China 19992122581272915272

\#\# 6 China 20002137661280428583

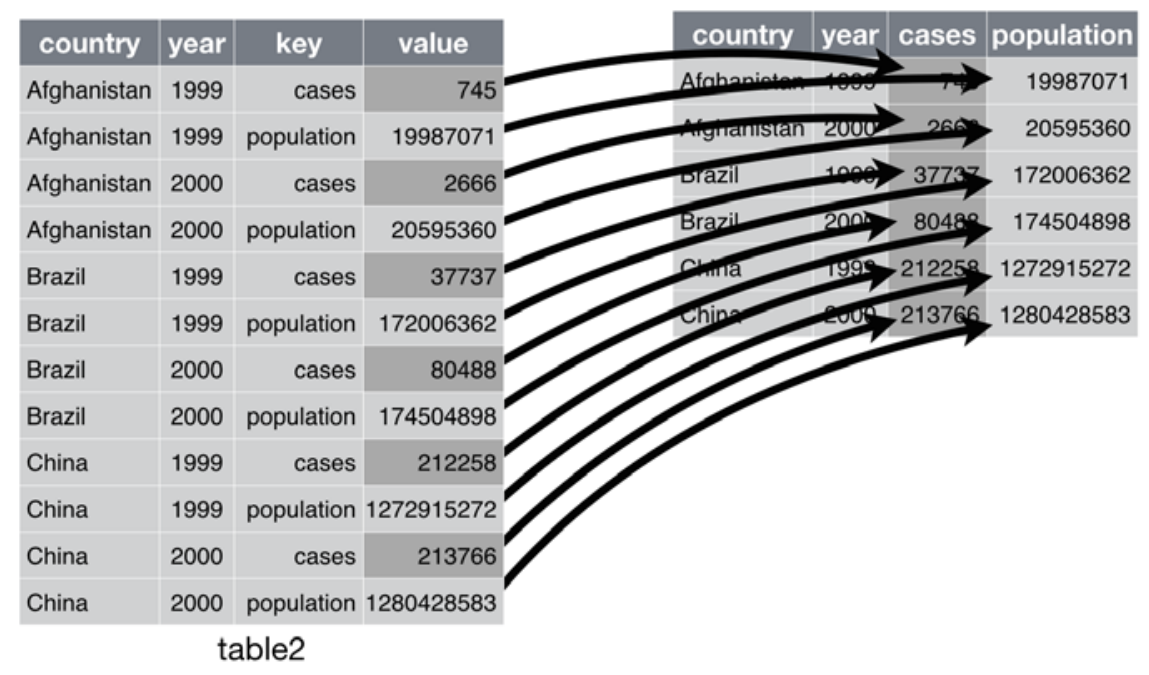

Figure 9.2 Spreading table2 makes it tidy

As you might have guessed from the common key and value arguments, spread() and gather() are complements. gather() makes wide tables narrower and longer; spread() makes long tables shorter and wider.

\subsubsection{Separating and Uniting}

So far, you've learned how to tidy table2 and table4, but not table3. table3 has a different problem: we have one column (rate) that contains two variables (cases and population). To fix this problem, we'll need the separate() function. You'll also learn about the complement of separate(): unite(), which you use if a single variable is spread across multiple columns. 


\subsubsection{Separate}

separate() pulls one column apart into multiple columns, by splitting wherever a separator character appears. Take table3:

table3

\#\# \# A tibble: 6 x 3

\#\# country year rate

\#\# $*$ <chr $><$ int $>\quad<$ chr $>$

\#\# 1 Afghanistan 1999 745/19987071

\#\# 2 Afghanistan 2000 2666/20595360

\#\# 3 Brazil 1999 37737/172006362

\#\# 4 Brazil $2000 \quad 80488 / 174504898$

\#\# 5 China 1999 212258/1272915272

\#\# 6 China 2000 213766/1280428583

The rate column contains both cases and population variables, and we need to split it into two variables. separate() takes the name of the column to separate, and the names of the columns to separate into, as shown in Figure 9.3 and the code below.

table3 \%>\%

separate(rate, into = c("cases", "population"))

\#\# \# A tibble: 6 x 4

\#\# country year cases population

\#\#* <chr $><$ int $><$ chr $>\quad<$ chr $>$

\#\# 1 Afghanistan 199974519987071

\#\# 2 Afghanistan 2000266620595360

\#\# 3 Brazil 199937737172006362

\#\# 4 Brazil 200080488174504898

\#\# 5 China 19992122581272915272

\#\# 6 China 20002137661280428583 


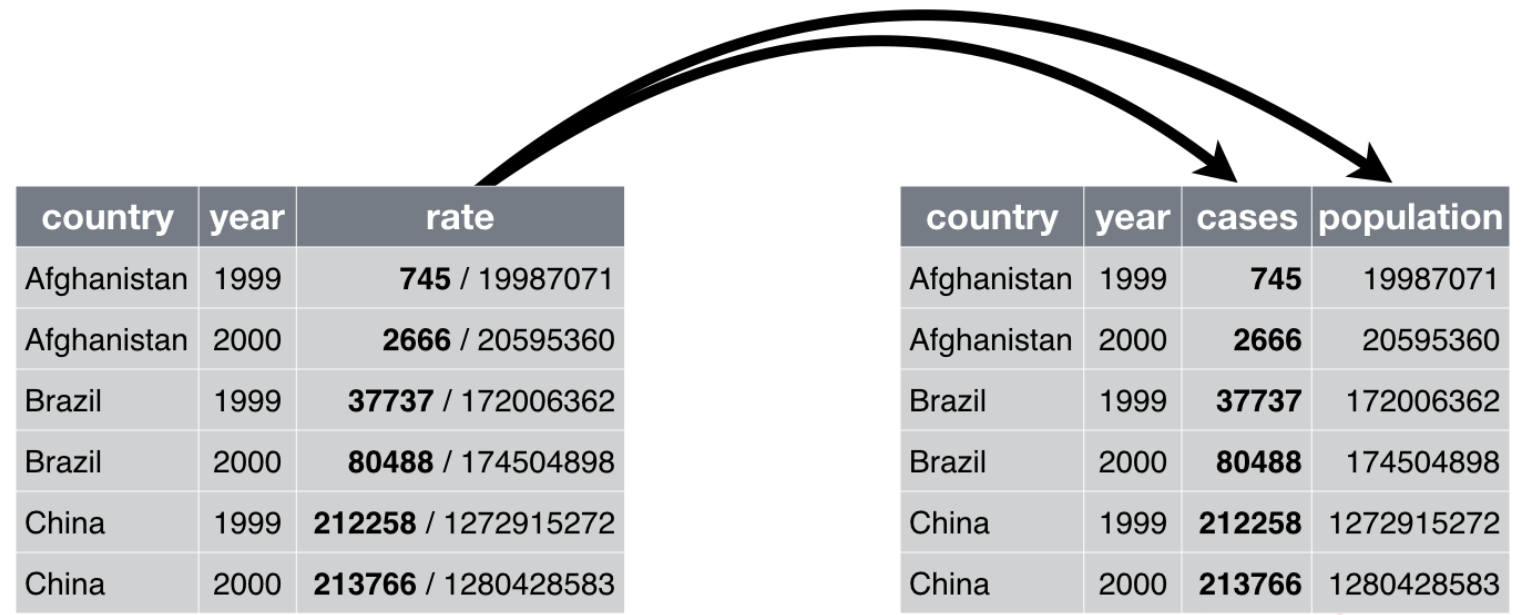

table3

Figure 9.3 Separating table3 makes it tidy

By default, separate() will split values wherever it sees a non-alphanumeric character (i.e., a character that isn't a number or letter). For example, in the code above, separate() split the values of rate at the slash characters. If you wish to use a specific character to separate a column, you can pass the character to the sep argument of separate(). For example, we could rewrite the code above as:

table3 \%>\%

separate(rate, into = c("cases", "population"), sep = "/")

(Formally, sep is a regular expression, which you can learn more about in the Strings chapter of R4DS.)

Look carefully at the column types: you'll notice that case and population are character columns. This is the default behavior in separate(): It leaves the type of the column as is. Here, however, it's not very useful as those really are numbers. We can ask separate() to try converting to better types using convert = TRUE:

table3 \%>\%

separate(rate, into = c("cases", "population"), convert = TRUE)

\#\# \# A tibble: 6 x 4

\#\# country year cases population

\#\# * $\quad<$ chr $><$ int $><$ int $>\quad<$ int $>$

\#\# 1 Afghanistan 199974519987071

\#\# 2 Afghanistan 2000266620595360

\#\# 3 Brazil 199937737172006362

\#\# 4 Brazil 200080488174504898 
You can also pass a vector of integers to sep. separate() will interpret the integers as positions to split at. Positive values start at 1 on the far left of the strings; negative values start at -1 on the far right of the strings. When using integers to separate strings, the length of sep should be one less than the number of names in into.

You can use this arrangement to separate the last two digits of each year. This makes these data less tidy, but is useful in other cases, as you'll see in a little bit.

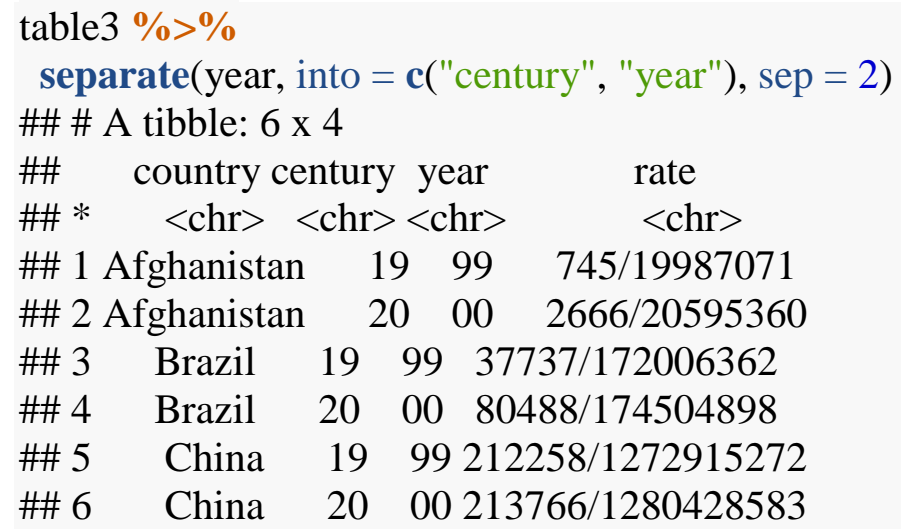

\subsubsection{Unite}

unite() is the inverse of separate(): it combines multiple columns into a single column. You'll need it much less frequently than separate(), but it's still a useful tool to have in your back pocket.

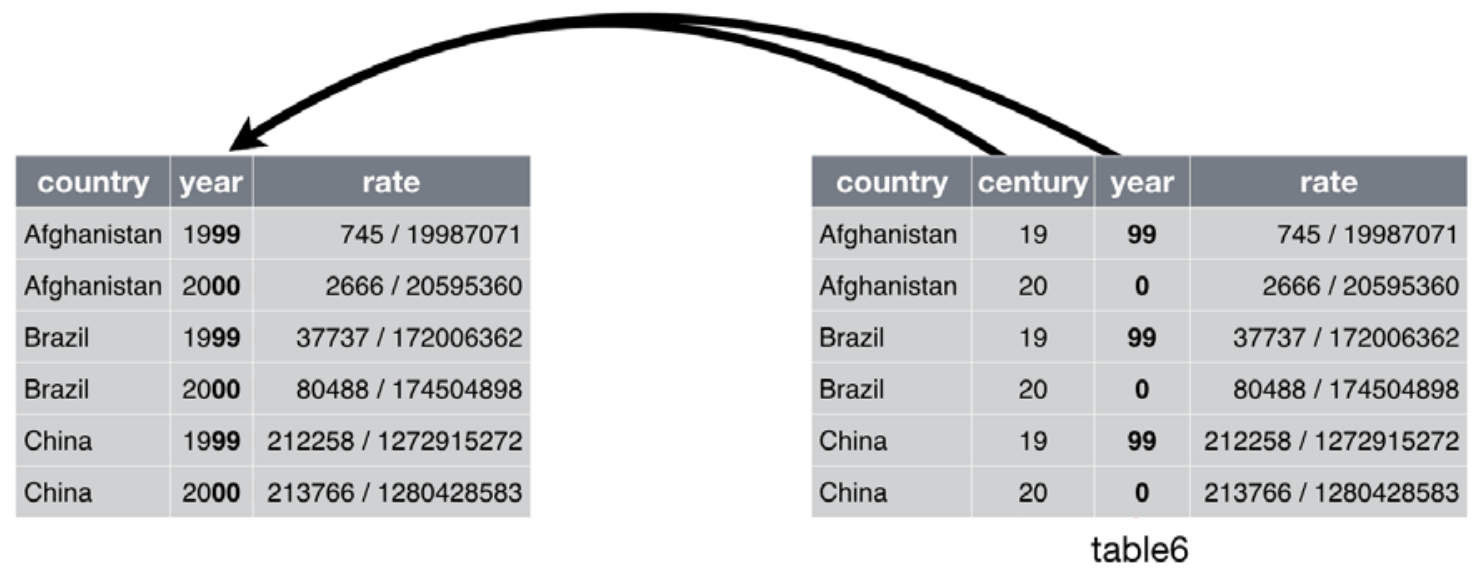

Figure 9.4 Uniting table5 makes it tidy 
We can use unite() to rejoin the century and year columns that we created in the last example (Figure 9.4). The data are saved as tidyr::table5. unite() takes a data frame, the name of the new variable to create, and a set of columns to combine, again specified in dplyr::select() style:

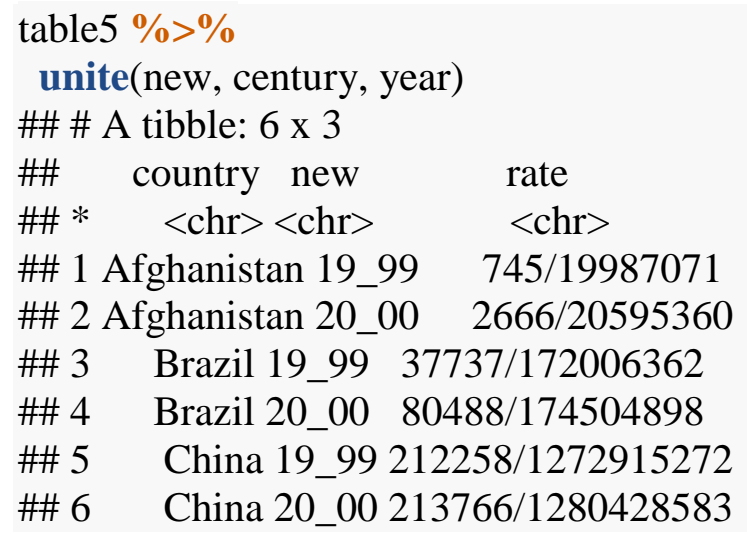

In this case, we also need to use the sep argument. The default will place an underscore (_) between the values from different columns. Here we don't want any separator so we use ":

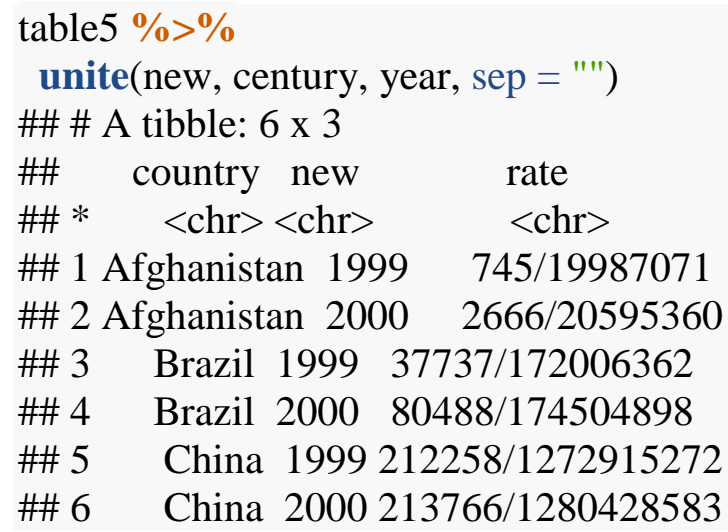

\subsection{EXERCISE}

1. Are the bike counts data for the two bridges tidy data?

2. If not, why not? And how can we tidy it?

3. After tidying the bike counts, using functions in the tidyr package, create tables summarizing the average bike counts by bridge and day of week in two different formats:

Table 9.1 Bike counts by day of week and bridge (1st format)

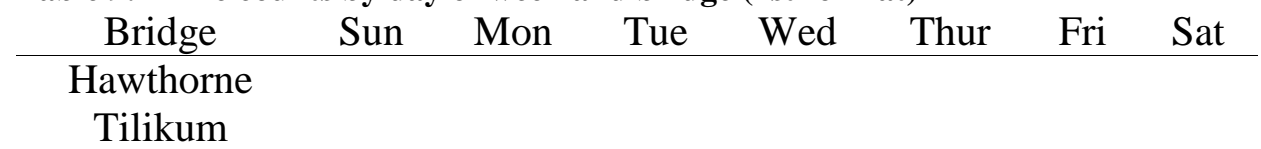

Table 9.2 Bike counts by day of week and bridge (2nd format)

\begin{tabular}{cc} 
Day of Week Hawthorne Tilikum \\
\hline Fri \\
Mon
\end{tabular}


Sat

Sun

Thur

Tue

Wed

\subsection{LEARNING MORE}

1. Dataframe Manipulation with tidyr

2. Data Wrangling Cheatsheet

3. Introduction to tidyr 


\subsection{DATA MANIPULATION WITH DPLYR}

\subsection{READINGS}

1. R4DS Data Transformation

2. R4DS Relational Data

\subsection{OVERVIEW}

dplyr is a grammar of data manipulation, providing a consistent set of verbs that helps you solve the most common data manipulation challenges:

- $\quad$ mutate() adds new variables that are functions of existing variables.

- $\quad$ select() picks variables based on their names.

- $\quad$ filter() picks cases based on their values.

- $\quad$ summarise() reduces multiple values down to a single summary.

- $\quad$ arrange() changes the ordering of the rows.

These all combine naturally with group_by() which allows you to perform any operation "by group." You can learn more about them in vignette("dplyr"). As well as these single-table verbs, dplyr also provides a variety of two-table verbs, which you can learn about in vignette("twotable").

dplyr is designed to abstract over how the data are stored. That means as well as working with local data frames, you can also work with remote database tables using exactly the same R code. Install the dbplyr package and then read vignette("databases", package = "dbplyr").

\subsection{LESSON}

The lesson below is adapted from UBC's Stat 545 course materials by Professor Jenny Bryan.

\subsubsection{Prerequisites}

Load dplyr (or tidyverse, which will load dplyr) and gapminder (install it if not yet installed):

library(gapminder)

library(tidyverse)

\subsubsection{Use filter() to Subset Data Row-wise}

filter() takes logical expressions and returns the rows for which all are TRUE.

filter(gapminder, lifeExp $<29$ )

\#\# \# A tibble: 2 x 6

\#\# country continent year lifeExp pop gdpPercap

\#\# $\quad<$ fctr $>\quad<$ fctr $><$ int $>\quad<$ dbl $>\quad<$ int $>\quad<$ dbl $>$ 
\#\# 1 Afghanistan Asia 195228.8018425333779 .4453

\#\# 2 Rwanda Africa 199223.5997290203737 .0686

filter(gapminder, country == "Rwanda", year > 1979)

\#\# \# A tibble: 6 × 6

\#\# country continent year lifeExp pop gdpPercap

\#\# <fctr $>\quad<$ fctr $><$ int $>\quad<$ dbl $><$ int $>\quad<$ dbl $>$

\#\# 1 Rwanda Africa 198246.2185507565881 .5706

\#\# 2 Rwanda Africa 198744.0206349365847 .9912

\#\# 3 Rwanda Africa 199223.5997290203737 .0686

\#\# 4 Rwanda Africa 199736.0877212583589 .9445

\#\# 5 Rwanda Africa 200243.4137852401785 .6538

\#\# 6 Rwanda Africa 200746.2428860588863 .0885

filter(gapminder, country \%in \% c("Rwanda", "Afghanistan"))

\#\# \# A tibble: 24 x 6

\#\# country continent year lifeExp pop gdpPercap

\#\# $\quad<$ fctr $>\quad<$ fctr $><$ int $>\quad<$ dbl $>\quad<$ int $><$ dbl $>$

\#\# 1 Afghanistan Asia 195228.8018425333779 .4453

\#\# 2 Afghanistan Asia 195730.3329240934820 .8530

\#\# 3 Afghanistan Asia 196231.99710267083853 .1007

\#\# 4 Afghanistan Asia 196734.02011537966836 .1971

\#\# 5 Afghanistan Asia 197236.08813079460739 .9811

\#\# 6 Afghanistan Asia 197738.43814880372786 .1134

\#\# 7 Afghanistan Asia 198239.85412881816978 .0114

\#\# 8 Afghanistan Asia 198740.82213867957852 .3959

\#\# 9 Afghanistan Asia 199241.67416317921649 .3414

\#\# 10 Afghanistan Asia 199741.76322227415635 .3414

\#\# \# ... with 14 more rows

Under no circumstances should you subset your data the way I did at first:

excerpt <- gapminder[241:252, ]

Why is this a terrible idea?

- It is not self-documenting. What is so special about rows 241 through 252?

- It is fragile. This line of code will produce different results if someone changes the row order of gapminder (e.g., sorts the data earlier in the script).

filter(gapminder, country == "Canada")

This call explains itself and is fairly robust.

\subsubsection{Use select() to Subset the Data on Variables or Columns}

Use select() to subset the data on variables or columns. Here's a conventional call: 
select(gapminder, year, lifeExp)

\#\# \# A tibble: 1,704 x 2

\#\# year lifeExp

\#\# <int> <dbl>

\#\# 1195228.801

\#\# 2195730.332

\#\# 3196231.997

\#\# 4196734.020

\#\# 5197236.088

\#\# 6197738.438

\#\# $7 \quad 198239.854$

\#\# 8198740.822

\#\# 9199241.674

\#\# 10199741.763

\#\# \# ... with 1,694 more rows

Think: "Take gapminder, then select the variables year and lifeExp, then show the first four rows."

\subsubsection{Revel in the Convenience}

Here are the data for Cambodia, taking certain variables:

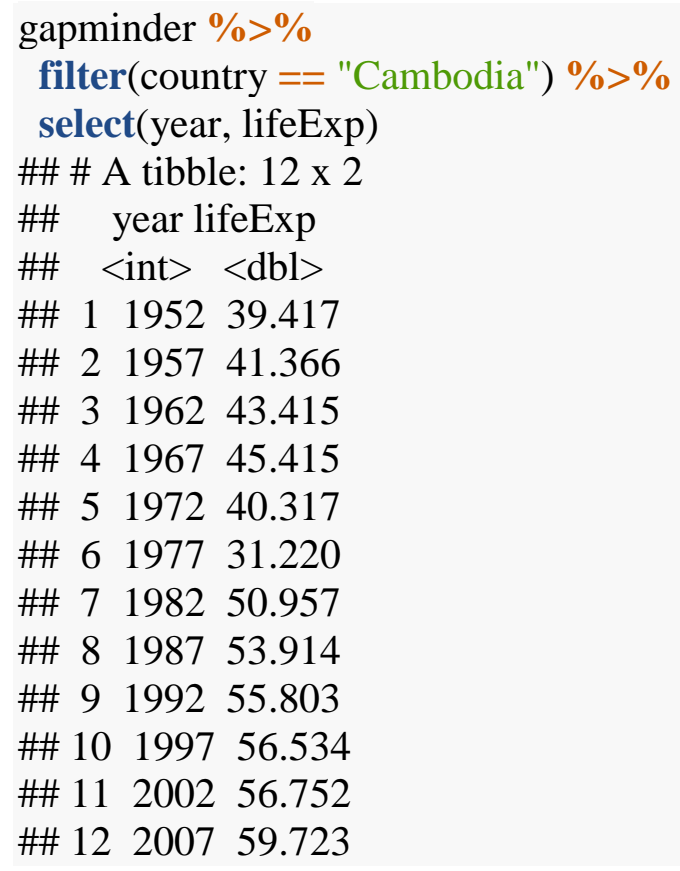

\subsubsection{Pure, Predictable, Pipeable}

We've barely scratched the surface of dplyr but I want to point out key principles you may start to appreciate. If you're new to R or "programming with data," feel free skip this section and move on. 
dplyr's verbs, such as filter() and select(), are what's called pure functions. To quote from Wickham's Advanced R Programming book:

The functions that are the easiest to understand and reason about are pure functions: functions that always map the same input to the same output and have no other impact on the workspace. In other words, pure functions have no side effects: they don't affect the state of the world in any way apart from the value they return.

In fact, these verbs are a special case of pure functions: they take the same flavor of the object as input and output. Namely, a data frame or one of the other data receptacles dplyr supports.

And finally, the data are always the very first argument of the verb functions.

This set of deliberate design choices, together with the new pipe operator, produces a highly effective, low friction domain-specific language for data analysis.

\subsubsection{Use mutate() to Add New Variables}

Imagine we wanted to recover each country's GDP. After all, the Gapminder data have a variable for population and GDP per capita. Let's multiply them together.

mutate() is a function that defines and inserts new variables into a tibble. You can refer to existing variables by name.

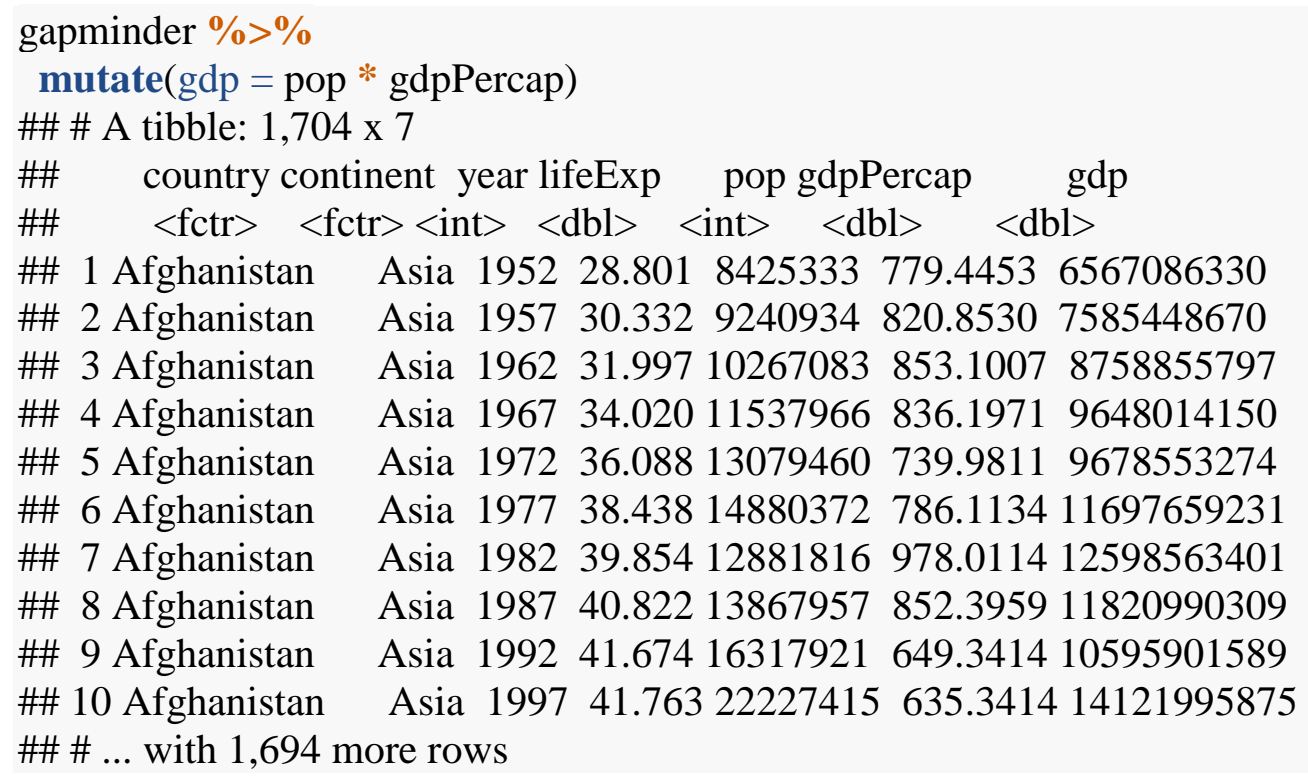

Hmm ... those GDP numbers are almost uselessly large and abstract. Consider the advice of Randall Munroe of xkcd:

One thing that bothers me is large numbers presented without context... 'If I added a zero to this number, would the sentence containing it mean something different to me?' If the answer is 'no,' maybe the number has no business being in the sentence in the first place." 
Maybe it would be more meaningful to consumers of my tables and figures to stick with GDP per capita. But what if I reported GDP per capita, relative to a benchmark country.

I need to create a new variable that is gdpPercap divided by Canadian gdpPercap, taking care that I always divide two numbers that pertain to the same year.

How I achieve this:

- $\quad$ Filter down to the rows for Canada

- $\quad$ Create a new temporary variable in gapminder:

- $\quad$ Extract the gdpPercap variable from the Canadian data.

- $\quad$ Replicate it once per country in the dataset, so it has the right length.

- $\quad$ Divide raw gdpPercap by this Canadian figure.

- Discard the temporary variable of replicated Canadian gdpPercap.

ctib $<$ - gapminder $\%>\%$

filter(country $==$ "Canada") $\%>\%$

select(year, BasegdpPercap=gdpPercap)

ctib

\#\# \# A tibble: 12 x 2

\#\# year BasegdpPercap

\#\# <int> <dbl>

\#\# $11952 \quad 11367.16$

\#\# $21957 \quad 12489.95$

\#\# $31962 \quad 13462.49$

\#\# $41967 \quad 16076.59$

\#\# $51972 \quad 18970.57$

\#\# $61977 \quad 22090.88$

\#\# $71982 \quad 22898.79$

\#\# $81987 \quad 26626.52$

\#\# $91992 \quad 26342.88$

\#\# $101997 \quad 28954.93$

\#\# $11 \quad 2002 \quad 33328.97$

\#\# $122007 \quad 36319.24$

my_gap <- gapminder \% $\% \%$

inner_join(ctib, by="year") \%>\%

mutate(gdpPercapRel = gdpPercap $/$ BasegdpPercap,

BasegdpPercap $=$ NULL)

my_gap

\#\# \# A tibble: 1,704 x 7

\#\# country continent year lifeExp pop gdpPercap gdpPercapRel

\#\# $\quad<$ fctr $><$ fctr $><$ int $><$ dbl $>\quad<$ int $>\quad<$ dbl $>\quad<$ dbl $>$

\#\# 1 Afghanistan Asia 195228.8018425333779 .44530 .06856992

\#\# 2 Afghanistan Asia $195730.3329240934820 .8530 \quad 0.06572108$

\#\# 3 Afghanistan Asia $196231.99710267083853 .1007 \quad 0.06336874$ 


$\begin{array}{lllllll}\text { \#\# 4 Afghanistan } & \text { Asia } 1967 & 34.02011537966 & 836.1971 & 0.05201335 \\ \text { \#\# 5 Afghanistan } & \text { Asia } 1972 & 36.088 & 13079460 & 739.9811 & 0.03900679 \\ \text { \#\# 6 Afghanistan } & \text { Asia } 1977 & 38.438 & 14880372 & 786.1134 & 0.03558542 \\ \text { \#\# 7 Afghanistan } & \text { Asia } 1982 & 39.85412881816 & 978.0114 & 0.04271018 \\ \text { \#\# 8 Afghanistan } & \text { Asia } 1987 & 40.822 & 13867957 & 852.3959 & 0.03201305 \\ \text { \#\# 9 Afghanistan } & \text { Asia } 1992 & 41.67416317921 & 649.3414 & 0.02464959 \\ \text { \#\# 10 Afghanistan } & \text { Asia } 1997 & 41.76322227415 & 635.3414 & 0.02194243 \\ \text { \#\# \# ... with 1,694 more rows } & & & \end{array}$

Note that, mutate() builds new variables sequentially so you can reference earlier ones (like tmp) when defining later ones (like gdpPercapRel). Also, you can get rid of a variable by setting it to NULL.

How could we sanity check that this worked? The Canadian values for gdpPercapRel better all be 1 !

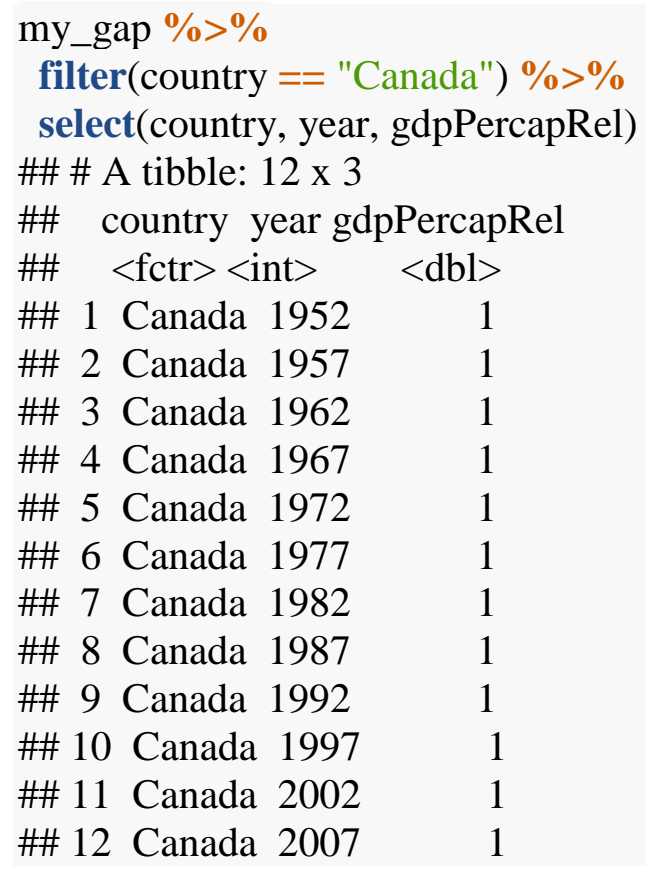

I perceive Canada to be a "high GDP" country, so I predict that the distribution of gdpPercapRel is located below 1, possibly even well below. Check your intuition!

summary(my_gap\$gdpPercapRel)

\#\# Min. 1st Qu. Median Mean 3rd Qu. Max.

\#\# 0.0072360 .0616480 .1715210 .3266590 .4465649 .534690

The relative GDP per capita numbers are, in general, well below 1 . We see that most of the countries covered by this dataset have substantially lower GDP per capita, relative to Canada, across the entire time period. 
Remember: Trust No One. Including (especially?) yourself. Always try to find a way to check that you've done what you meant to. Prepare to be horrified.

\subsubsection{Use arrange() to Row-order Data in a Principled Way}

arrange() reorders the rows in a data frame. Imagine you wanted these data ordered by year then country, as opposed to by country then year.

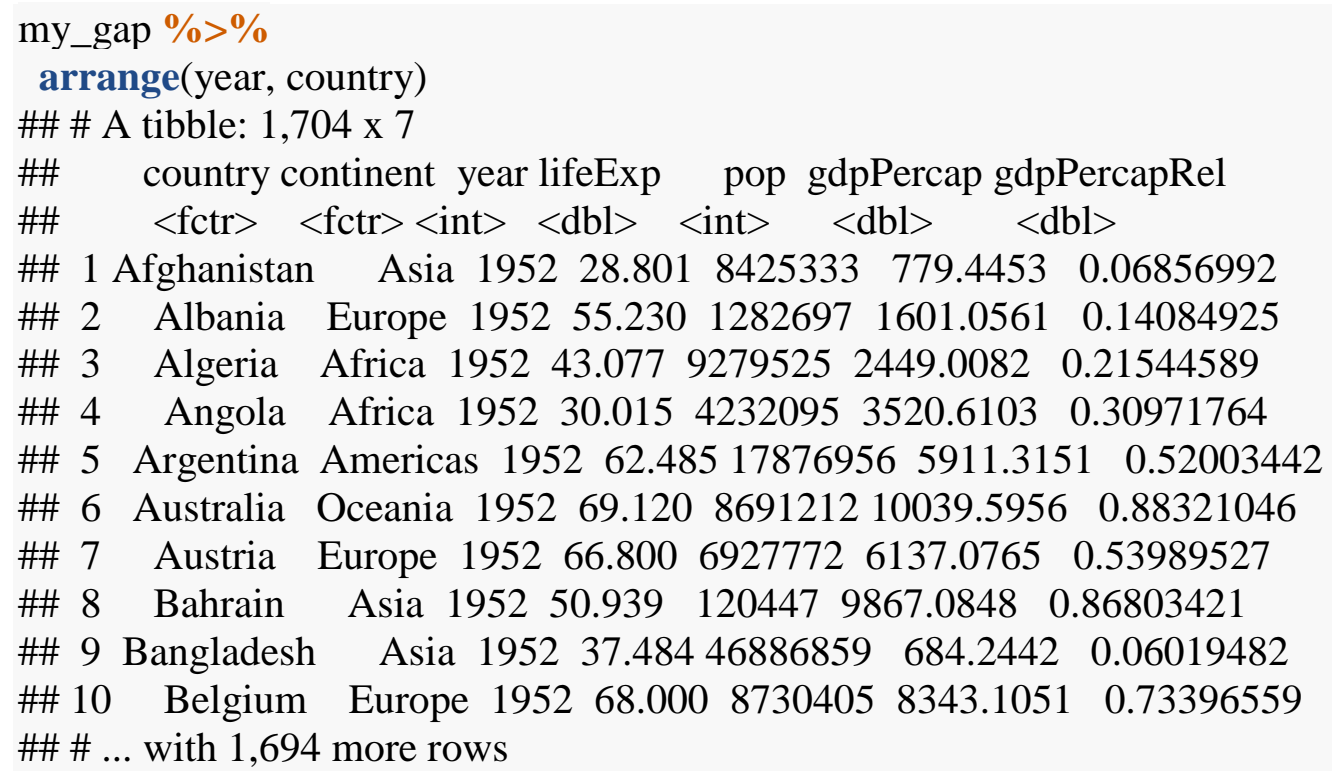

Or maybe you want just the data from 2007, sorted on life expectancy?

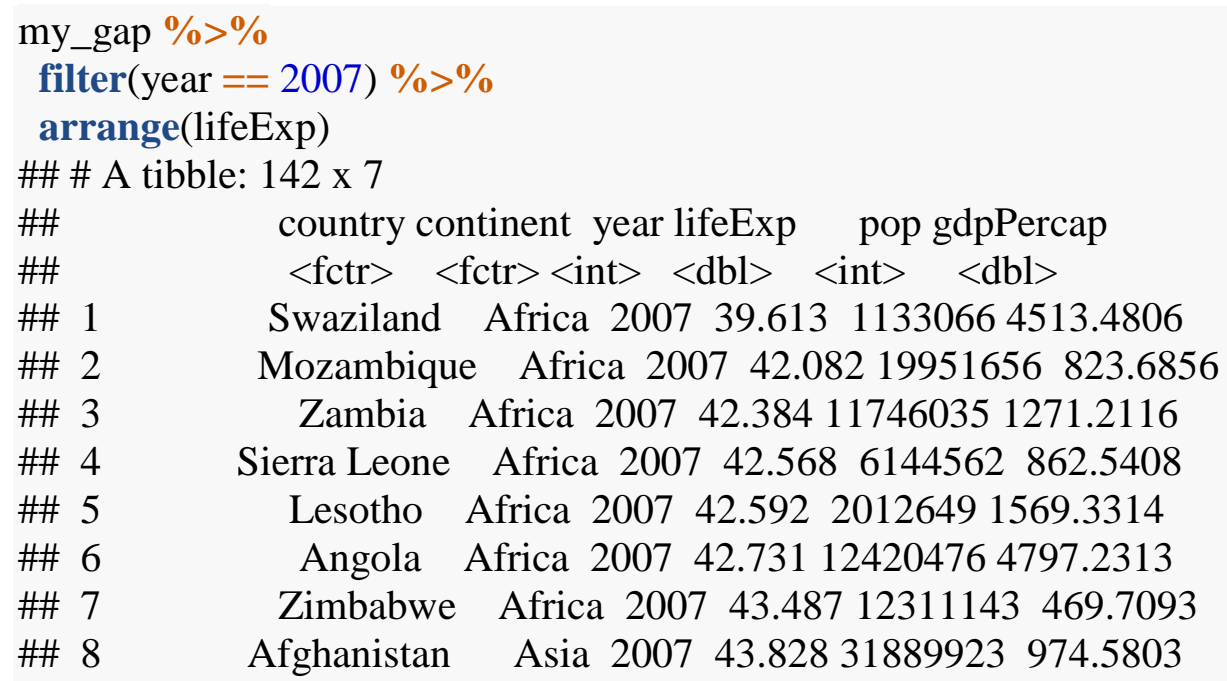




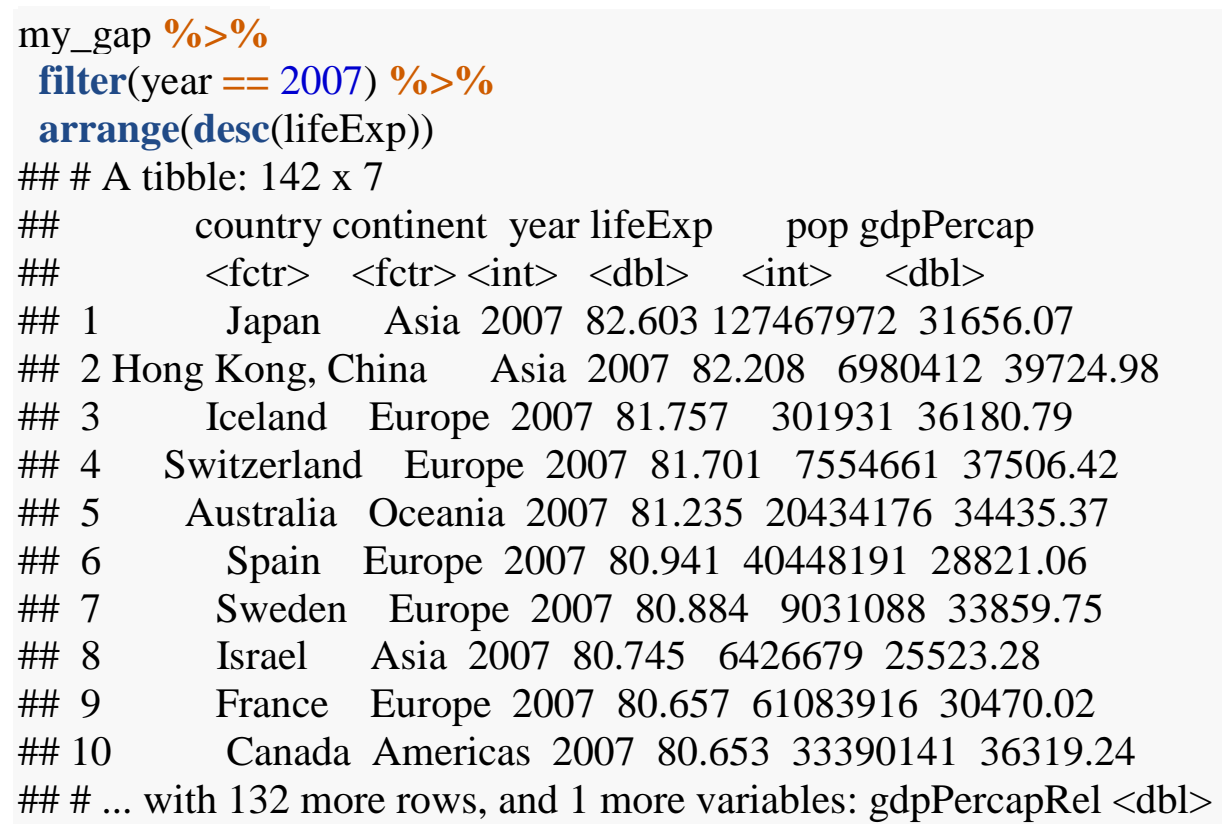

I advise that your analyses NEVER rely on rows or variables being in a specific order. But it's still true that human beings write the code and the interactive development process can be much nicer if you reorder the rows of your data as you go along. Also, once you are preparing tables for human eyeballs, it is imperative that you step up and take control of row order.

\subsubsection{Use rename() to Rename Variables}

The Gapminder uses camelCase for variable naming; an alternative convention is snake_case. Let's rename some variables to use snake_case!

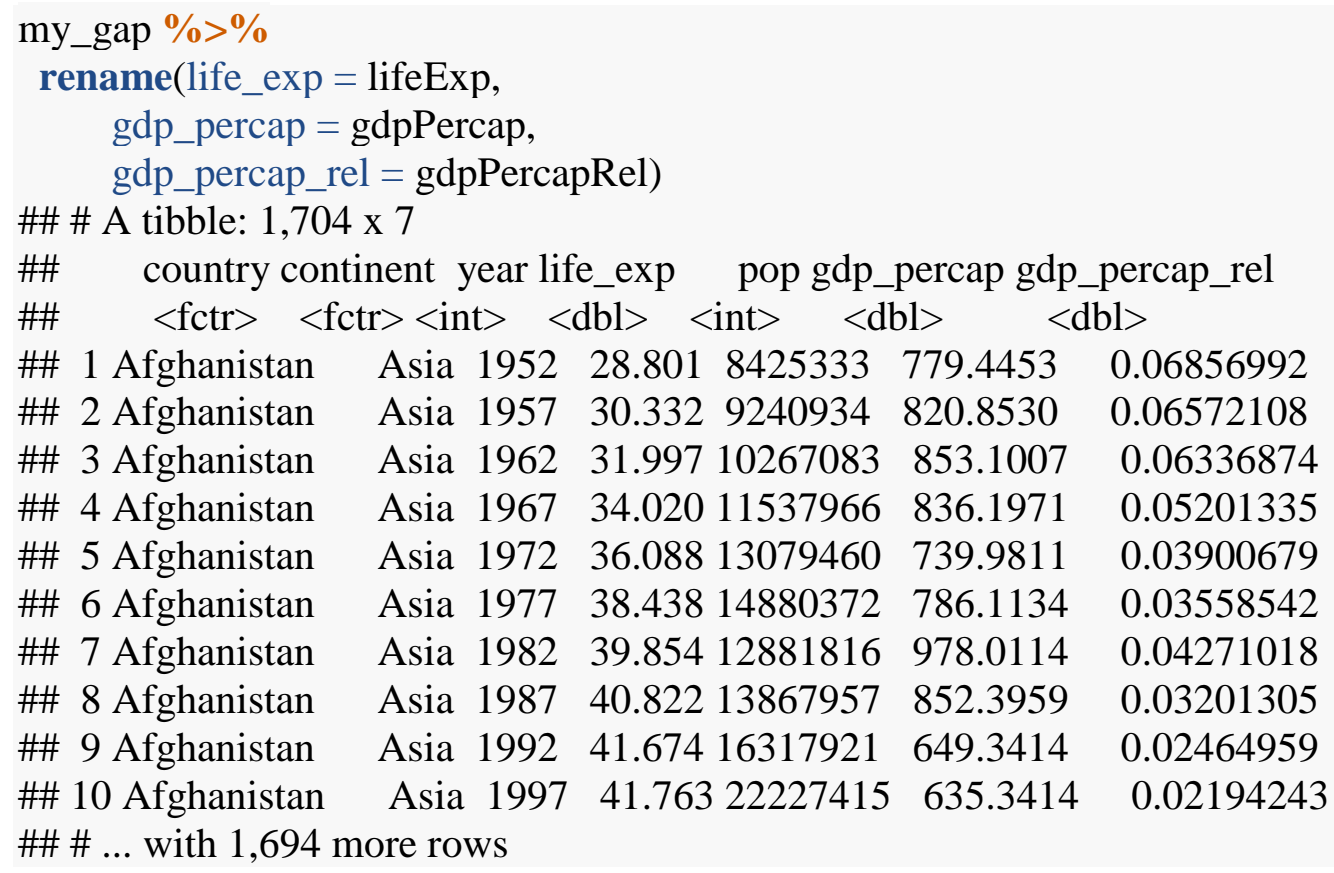


I did NOT assign the post-rename object back to my_gap because that would make the chunks in this tutorial harder to copy/paste and run out of order. In real life, I would probably assign this back to my_gap, in a data preparation script, and proceed with the new variable names.

\subsection{9 select() Can Rename and Reposition Variables}

You've seen simple uses of select(). There are two tricks you might enjoy:

1. select() can rename the variables you request to keep.

2. select() can be used with everything() to hoist a variable up to the front of the tibble.

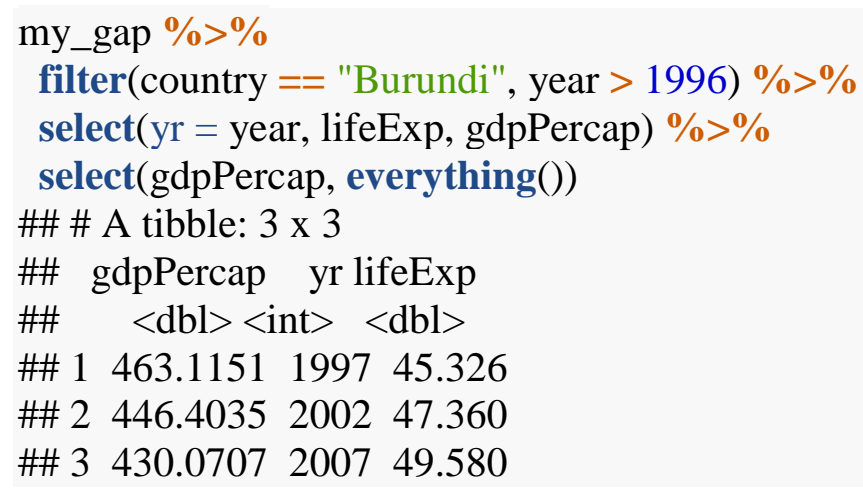

everything() is one of several helpers for variable selection. Read its help feature to see the rest.

\subsubsection{0 group_by() is a Mighty Weapon}

I have found that collaborators love to ask seemingly innocuous questions like, "Which country experienced the sharpest five-year drop in life expectancy?" In fact, that is a totally natural question to ask. But if you are using a language that doesn't know about data, it's an incredibly annoying question to answer.

dplyr offers powerful tools to solve this class of problem.

- group_by() adds extra structure to your dataset — grouping information — which lays the groundwork for computations within the groups.

- $\quad$ summarize() takes a dataset with $n$ observations, computes requested summaries, and returns a dataset with one observation.

- Window functions take a dataset with $n$ observations and returns a dataset with $n$ observations.

- $\quad$ mutate() and summarize() will honor groups.

- You can also do very general computations on your groups with do(), though elsewhere in this course I advocate for other approaches that I find more intuitive, using the purrr package.

Combined with the verbs you already know, these new tools allow you to solve an extremely diverse set of problems with relative ease. 


\subsubsection{Counting Things Up}

Let's start with simple counting. How many observations do we have per continent?

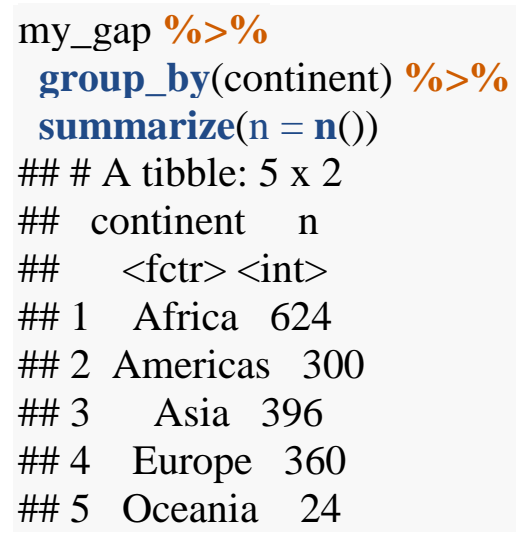

Let us pause here to think about the tidyverse. You could get these same frequencies using table() from base R.

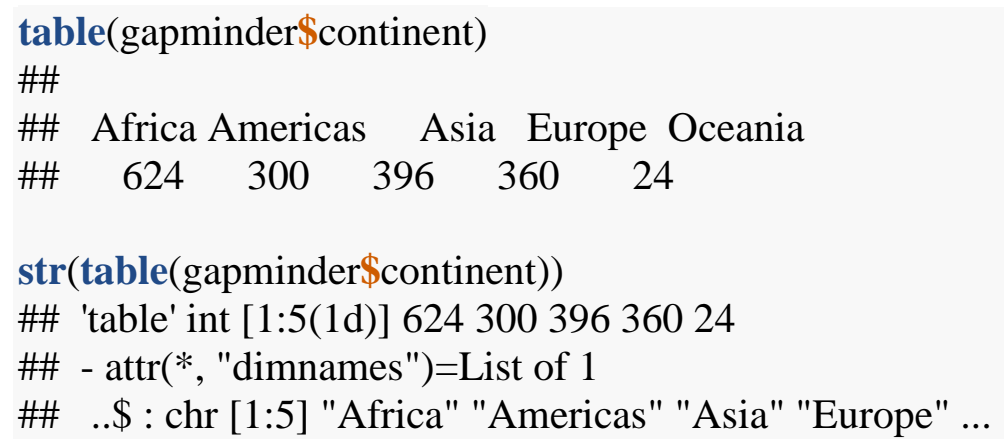

But the object of the class table that is returned makes downstream computation a bit fiddlier than you'd like. For example, it's too bad the continent levels come back only as names and not as a proper factor, with the original set of levels. This is an example of how the tidyverse smooths transitions where you want the output of step i to become the input of step i +1 .

The tally() function is a convenient function that knows to count rows. It honors groups.

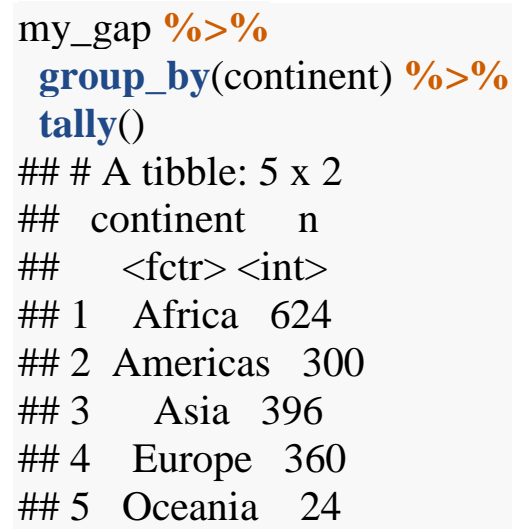


The count() function is an even more convenient function that does both grouping and counting.

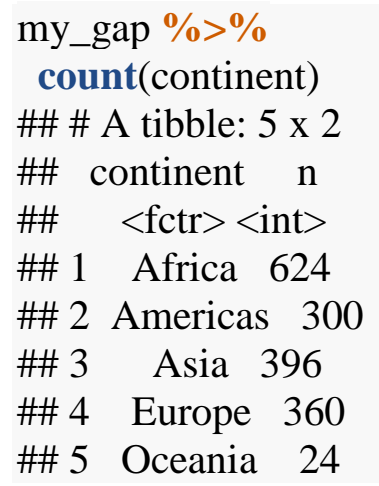

What if we wanted to add the number of unique countries for each continent? You can compute multiple summaries inside summarize(). Use the $\mathrm{n} \_$distinct() function to count the number of distinct countries within each continent.

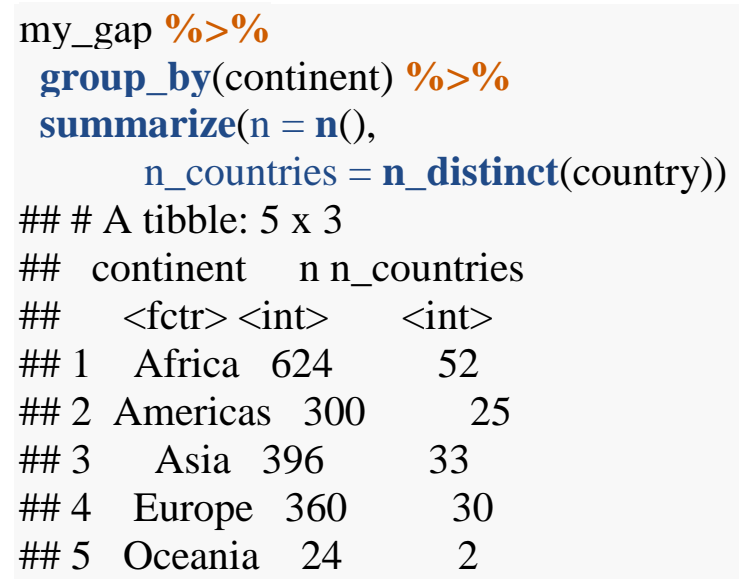

\subsubsection{General Summarization}

The functions you'll apply within summarize() include classical statistical summaries, like mean(), median(), $\operatorname{var}(), \operatorname{sd}(), \operatorname{mad}(), \mathrm{IQR}()$, $\min ($ ), and $\max ()$. Remember they are functions that take $n$ inputs and distill them down into one output.

Although this may be statistically ill-advised, let's compute the average life expectancy by continent.

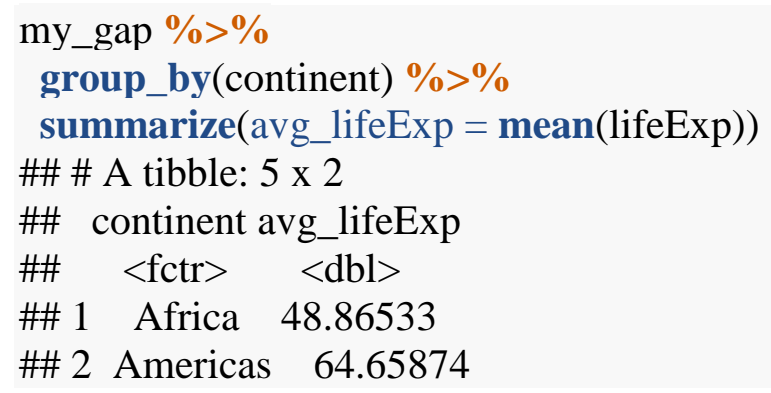


\#\# 3 Asia 60.06490

\#\# 4 Europe 71.90369

\#\# 5 Oceania 74.32621

summarize_each() applies the same summary function(s) to multiple variables. Let's compute average and median life expectancy and GDP per capita by continent by year ... but only for 1952 and 2007.

my_gap $\%>\%$

filter(year \% in \% c(1952, 2007)) \%>\%

group_by(continent, year) \%>\%

summarise_each(funs(mean, median), lifeExp, gdpPercap)

\#\# \# A tibble: 10 x 6

\#\# \# Groups: continent [?]

\#\# continent year lifeExp_mean gdpPercap_mean lifeExp_median

\#\# $\quad<$ fctr $><$ int $>\quad<$ dbl $>\quad<$ dbl $><$ dbl $>$

\#\# 1 Africa $1952 \quad 39.13550 \quad 1252.572 \quad 38.8330$

\#\# 2 Africa $2007 \quad 54.80604 \quad 3089.033 \quad 52.9265$

\#\# 3 Americas $1952 \quad 53.27984 \quad 4079.063 \quad 54.7450$

\#\# 4 Americas $2007 \quad 73.60812 \quad 11003.032 \quad 72.8990$

$\begin{array}{lllll}\text { \#\# } 5 & \text { Asia } 1952 & 46.31439 & 5195.484 & 44.8690\end{array}$

\#\# 6 Asia $2007 \quad 70.72848 \quad 12473.027 \quad 72.3960$

\#\# 7 Europe $1952 \quad 64.40850 \quad 5661.057 \quad 65.9000$

\#\# 8 Europe $2007 \quad 77.64860 \quad 25054.482 \quad 78.6085$

\#\# 9 Oceania $1952 \quad 69.25500 \quad 10298.086 \quad 69.2550$

\#\# 10 Oceania $2007 \quad 80.71950 \quad 29810.188 \quad 80.7195$

\#\# \# ... with 1 more variables: gdpPercap_median <dbl>

Let's focus just on Asia. What are the minimum and maximum life expectancies seen by year?

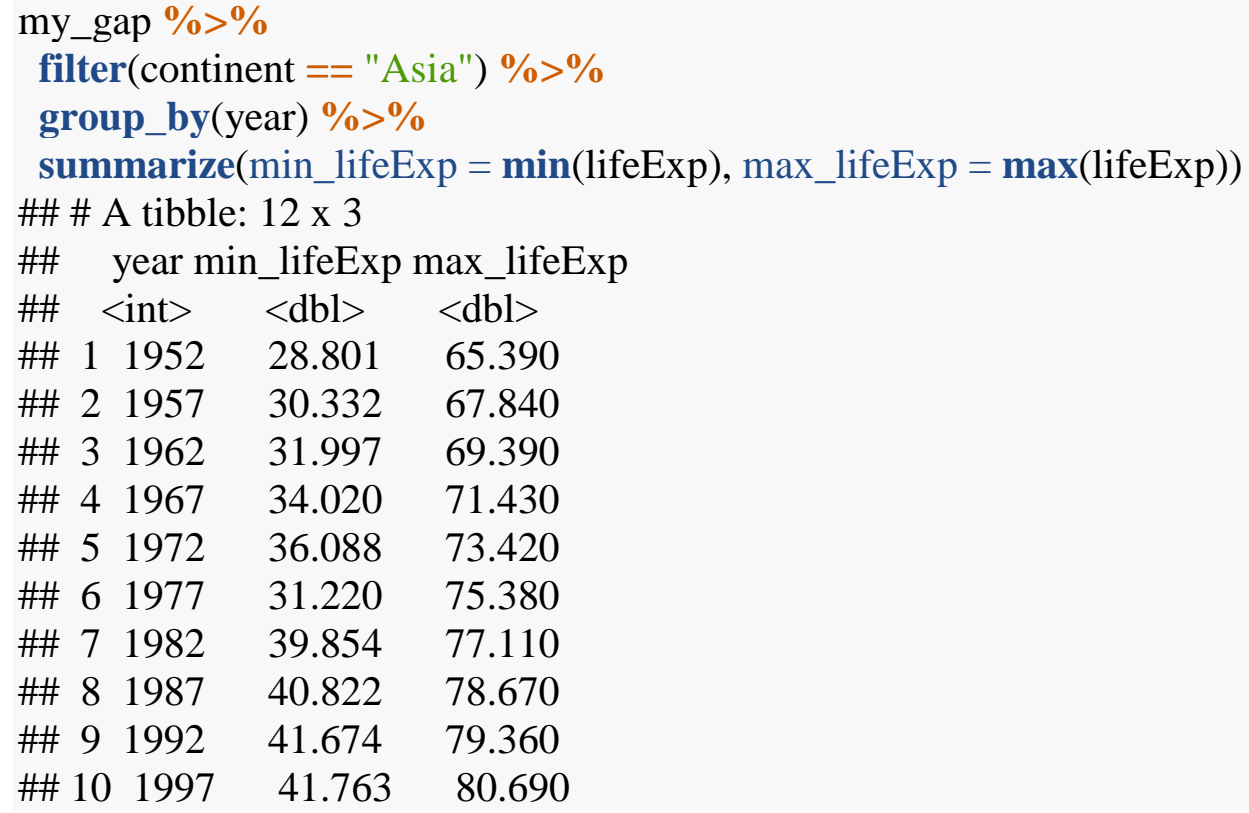


\#\# $112002 \quad 42.129 \quad 82.000$

\#\# $122007 \quad 43.828 \quad 82.603$

Of course, it would be much more interesting to see which country contributed these extreme observations. Is the minimum (maximum) always coming from the same country? We tackle that with window functions shortly.

\subsubsection{Grouped Mutate}

Sometimes you don't want to collapse the $n$ rows for each group into one row. You want to keep your groups, but compute within them.

\subsubsection{Computing With Group-wise Summaries}

Let's make a new variable that is the years of life expectancy gained (lost) relative to 1952, for each individual country. We group by country and use mutate() to make a new variable. The first() function extracts the first value from a vector. Notice that first() is operating on the vector of life expectancies within each country group.

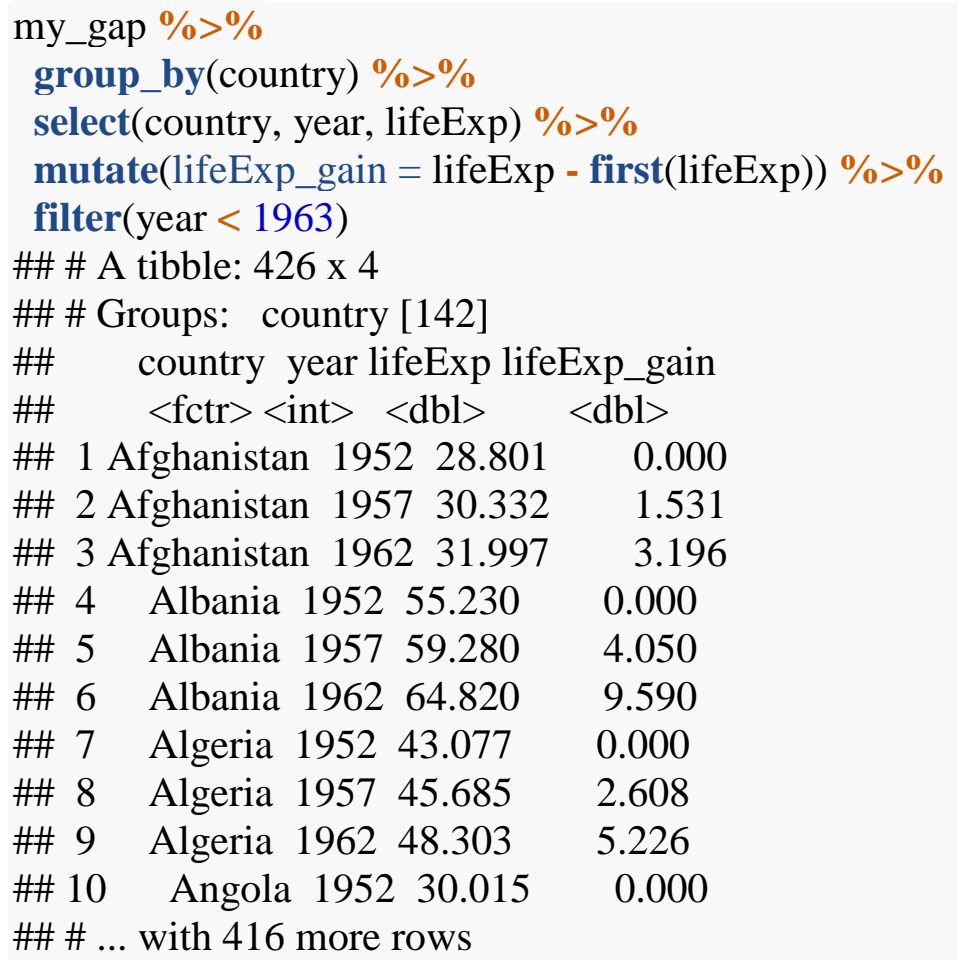

Within the country, we take the difference between life expectancy in year $i$ and life expectancy in 1952. Therefore, we always see zeroes for 1952 and, for most countries, a sequence of positive and increasing numbers. 


\subsubsection{Window Functions}

Window functions take $n$ inputs and give back $n$ outputs. Furthermore, the output depends on all the values. So $\operatorname{rank}()$ is a window function but $\log ()$ is not. Here we use window functions based on ranks and offsets.

Let's revisit the worst and best life expectancies in Asia over time, but retaining info about which country contributes these extreme values.

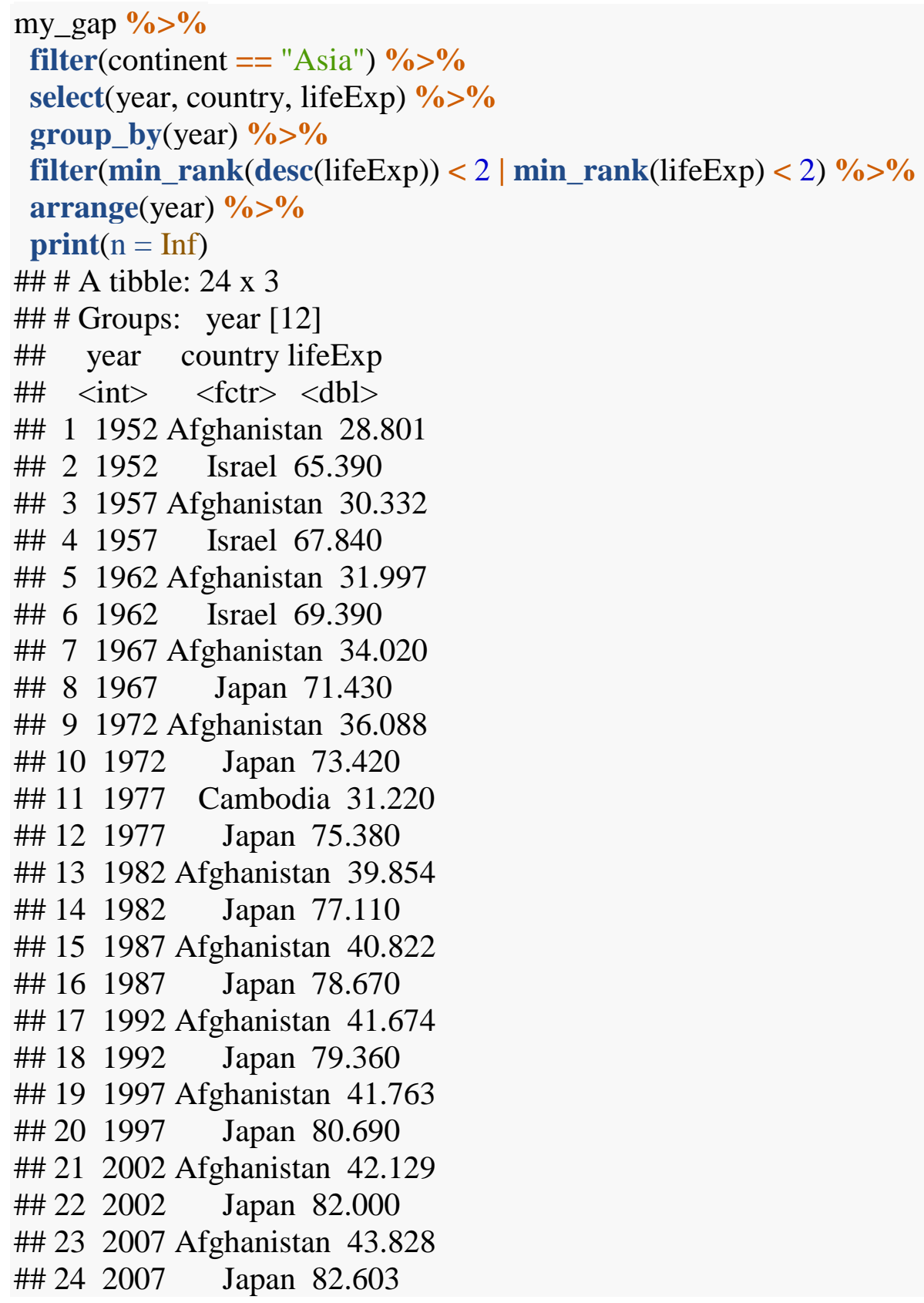

We see that $(\min =$ Afghanistan, $\max =$ Japan $)$ is the most frequent result, but Cambodia and Israel pop up at least once each as the min or max, respectively. That table should make you 
impatient for our upcoming work on tidying and reshaping data! Wouldn't it be nice to have one row per year?

How did that actually work? First, I store and view a partial that leaves off the filter() statement. All of these operations should be familiar.

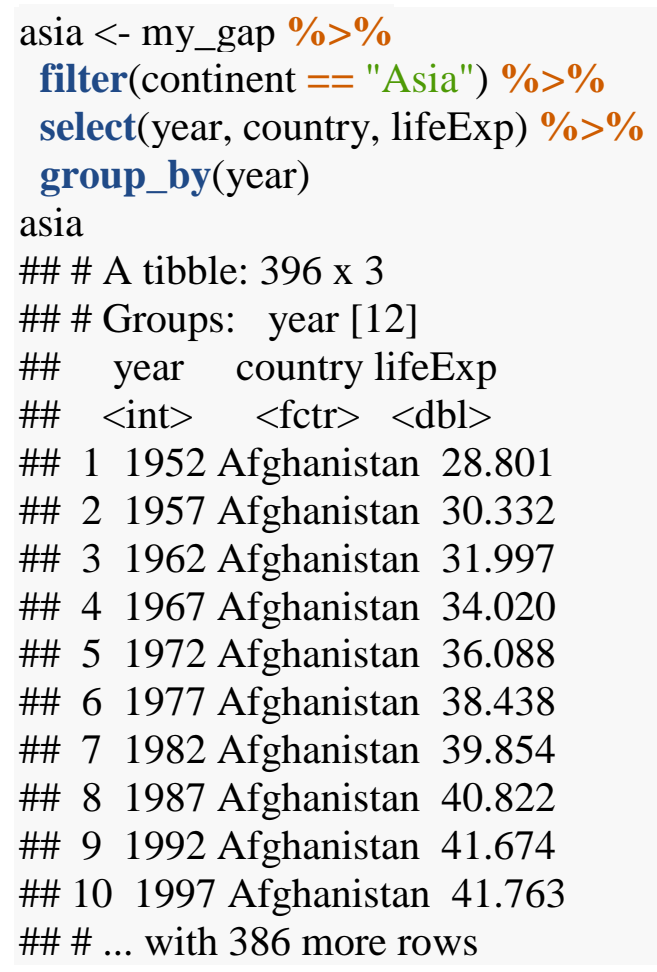

Now we apply a window function — min_rank(). Since Asia is grouped by year, min_rank() operates within mini-datasets, each for a specific year. Applied to the variable lifeExp, min_rank() returns the rank of each country's observed life expectancy. FYI, the min part just specifies how ties are broken. Here is an explicit peek at these within-year life expectancy ranks, in both the (default) ascending and descending order.

For concreteness, I use mutate() to actually create these variables, even though I dropped this in the solution above. Let's look at a bit of that.

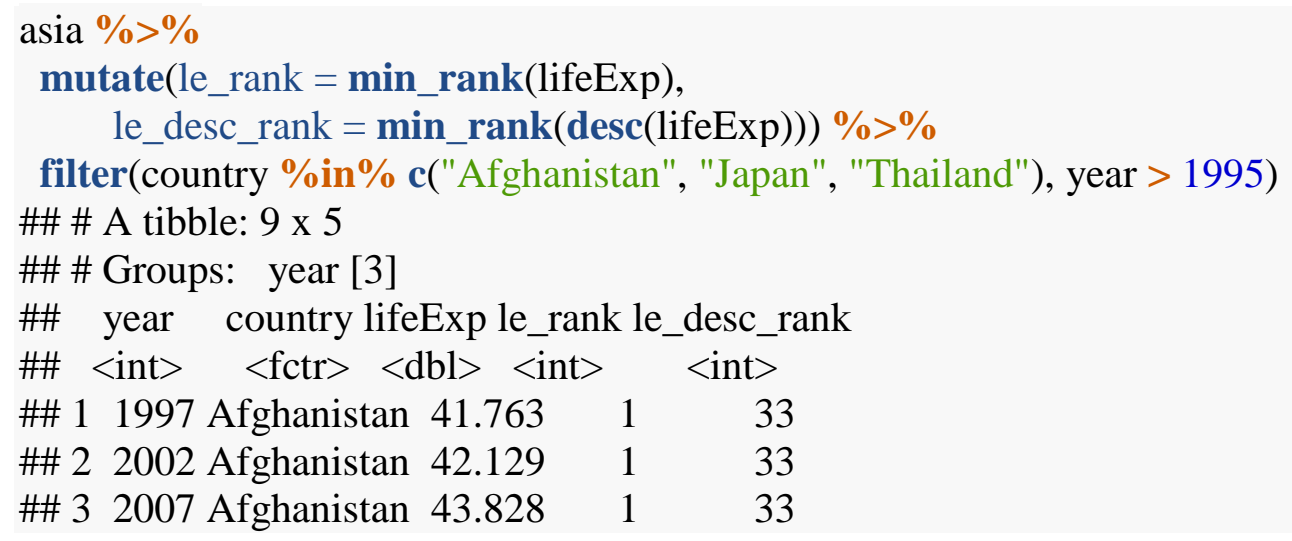




$\begin{array}{llccc}\text { \#\# 4 } & 1997 & \text { Japan 80.690 } & 33 & 1 \\ \text { \#\# 5 } & 2002 & \text { Japan 82.000 } & 33 & 1 \\ \text { \#\# 6 } & 2007 & \text { Japan 82.603 } & 33 & 1 \\ \text { \#\# 7 } & 1997 & \text { Thailand 67.521 } & 12 & 22 \\ \text { \#\# 8 } 2002 & \text { Thailand 68.564 } & 12 & 22 \\ \text { \#\# 9 } 2007 & \text { Thailand 70.616 } & 12 & 22\end{array}$

Afghanistan tends to present $1 \mathrm{~s}$ in the le_rank variable, Japan tends to present $1 \mathrm{~s}$ in the le_desc_rank variable and other countries, like Thailand, present less extreme ranks.

You can understand the original filter() statement now:

filter(min_rank(desc(lifeExp) $)<2 \mid$ min_rank(lifeExp) $<2$ )

These two sets of ranks are formed on the fly, within year group, and filter() retains rows with rank less than 2 , which means ... the row with rank $=1$. Since we do this for ascending and descending ranks, we get both the min and the max.

If we had wanted just the min OR the max, an alternative approach using top_n() would have worked.

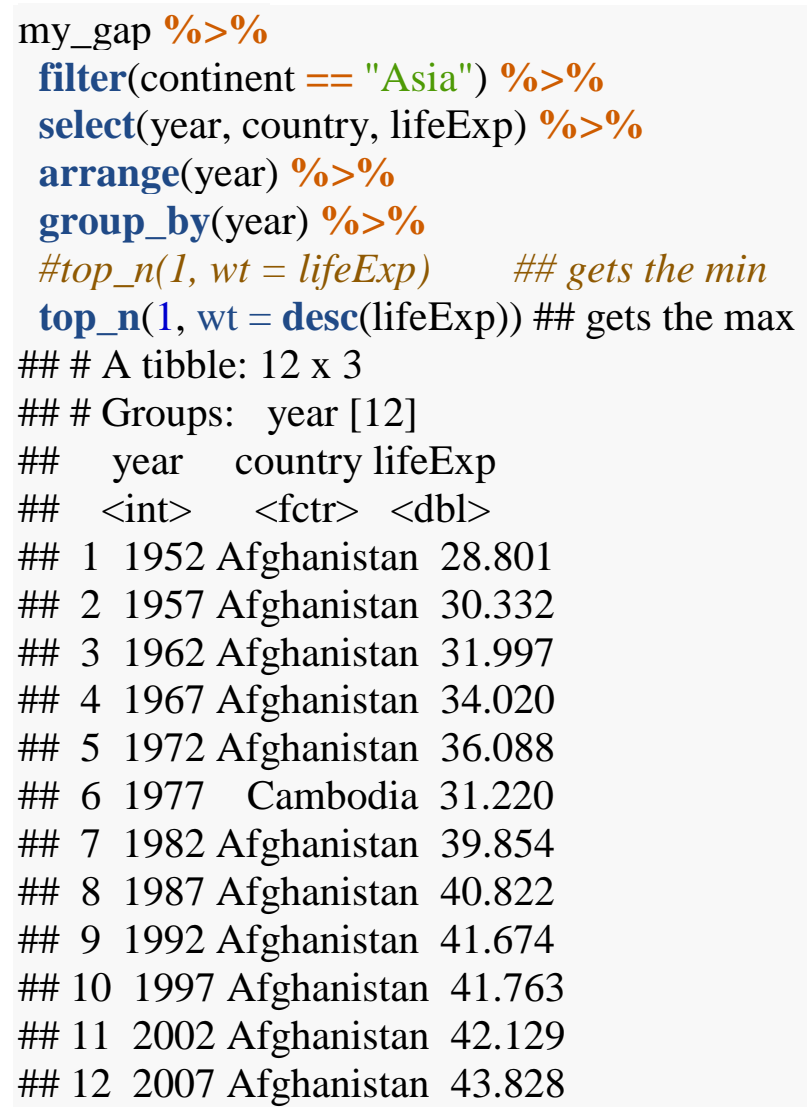




\subsubsection{Grand Finale}

So let's answer that "simple" question: Which country experienced the sharpest five-year drop in life expectancy? Recall that this excerpt of the Gapminder data only have data every five years (e.g., for 1952, 1957, etc.). So, this really means looking at life expectancy changes between adjacent time points.

At this point that's just too easy, so let's do it by continent while we're at it.

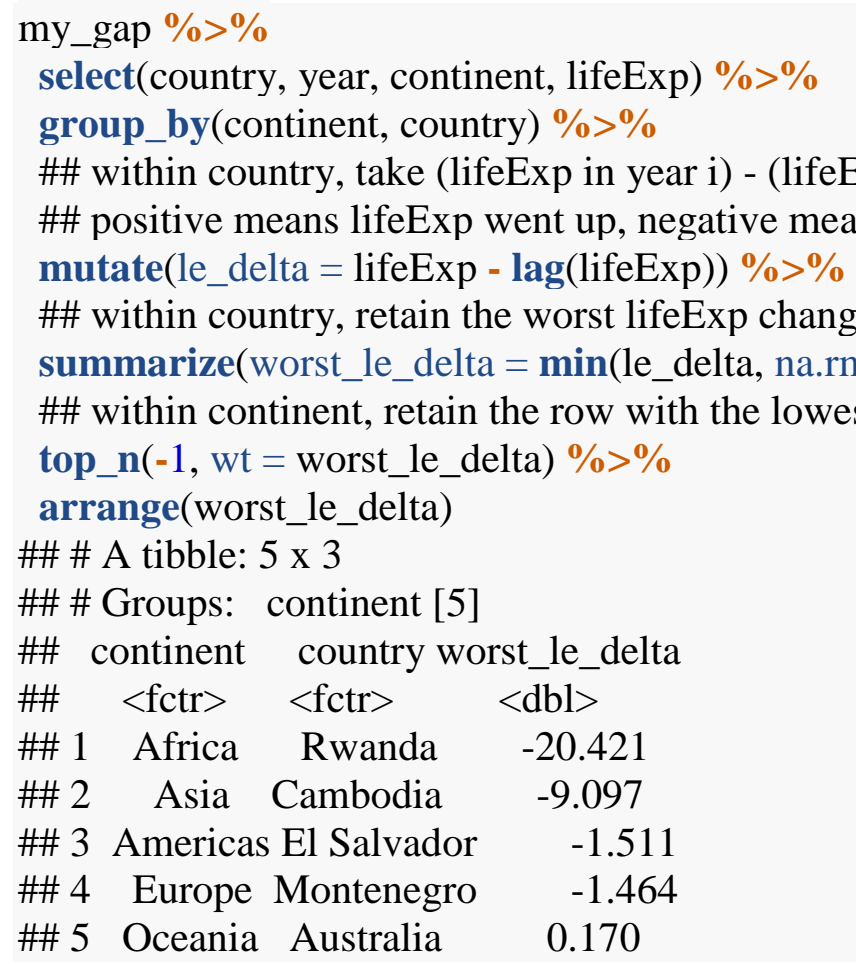

Ponder that for a while. The subject matter and the code. Mostly you're seeing what genocide looks like in dry statistics on average life expectancy.

Break the code into pieces, starting at the top, and inspect the intermediate results. That's certainly how I was able to write such a thing. These commands do not leap fully formed out of anyone's forehead - they are built up gradually, with lots of errors and refinements along the way. I'm not even sure it's a great idea to do so much manipulation in one fell swoop. Is the statement above really hard for you to read? If yes, then, by all means, break it into pieces and make some intermediate objects. Your code should be easy to write and read when you're done.

\subsubsection{Working With Two Datasets}

When working with two or more datasets, we routinely join them. dplyr adopts the SQL-dialect for joining datasets. Below are examples for those of us who don't speak SQL so well. There are lots of Venn diagrams re SQL joins on the interwebs, but I wanted R examples.

Full documentation for the dplyr package, which is developed by Hadley Wickham and Romain Francois on GitHub. The vignette on Two-table verbs covers the joins shown here. 


\subsubsection{Variable Recoding With dplyr}

- $\quad$ recode and recode_factor: Replace numeric values based on their position, and character values by their name;

- $\quad$ if_else: Replace values based on a logical vector; and

- $\quad$ case_when: Vectorise multiple if and else if statements.

Recoding, when to use which function: - one-to-one, many-to-one: recode and recode_factor Download the NHTS 2009 data file for the demos here (Right click and select Save As...)

library(tidyverse)

\# load NHTS2009 travel diaries subset

dd <-read_csv("data/NHTS2009_dd.csv")

\# recode race (HH_RACE column) according to data dictionary: http://nhts.ornl.gov/tables09/C odebookPage.aspx?id=951

dd $\%>\%$ mutate(hh_race_str=recode(HH_RACE,

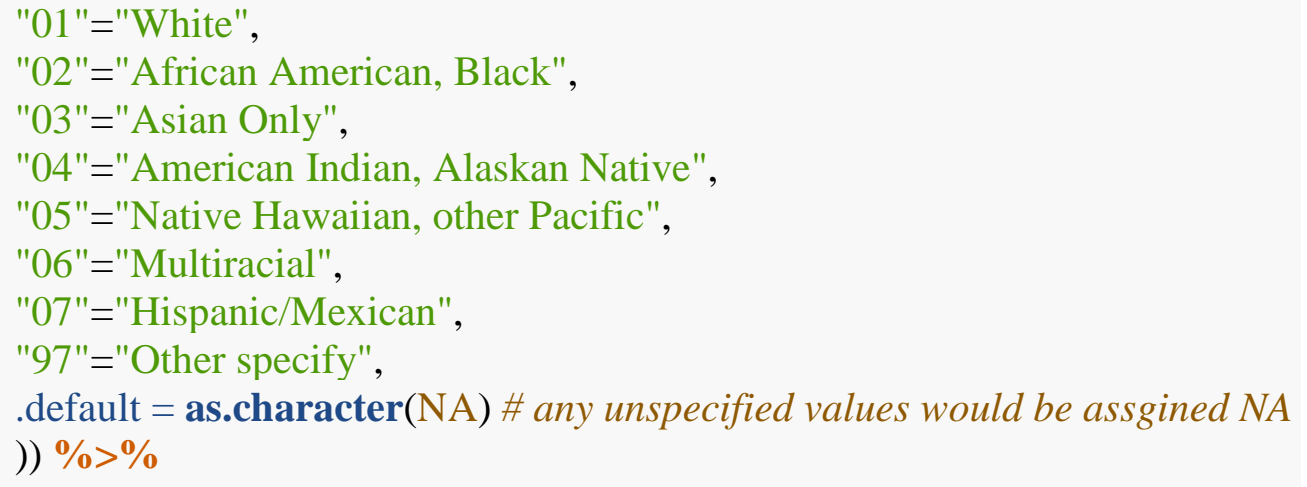

select(HH_RACE, hh_race_str)

\#\# \# A tibble: 304 x 2

\#\# HH_RACE hh_race_str

\#\# <chr $>\quad<$ chr $>$

\#\# $101 \quad$ White

\#\# $201 \quad$ White

\#\# $301 \quad$ White

\#\# 401 White

\#\# $501 \quad$ White

\#\# $601 \quad$ White

\#\# $701 \quad$ White

\#\# $801 \quad$ White

\#\# $901 \quad$ White

\#\# $1001 \quad$ White

\#\# \# ... with 294 more rows

- a logical condition: if_else 
\# code driving \& non-driving based on travel modes (TRPTRANS column) data dictionary: http:/ /nhts.ornl.gov/tables09/CodebookPage.aspx?id=1084

dd \%>\% mutate(driving=ifelse(TRPTRANS \% in \% c("01", "02", "03", "04", "05", "06", "07"), $1,0)$,

driving=ifelse(TRPTRANS \% in\% c("-1", "-7", "-8", "-9"), NA, driving) \# retain missin $g$ values as $N A$

$$
\text { ) } \%>\%
$$

select(TRPTRANS, driving)

\#\# \# A tibble: $304 \times 2$

\#\# TRPTRANS driving

\#\# <chr $><\mathrm{dbl}>$

\#\# 1 1 $03 \quad 1$

\#\# $2 \quad 03 \quad 1$

\#\# $3 \quad 03 \quad 1$

\#\# $4 \begin{array}{lll}4 & 03 & 1\end{array}$

\#\# $5 \quad 03 \quad 1$

\#\# $6 \quad 03 \quad 1$

\#\# $7 \quad 03 \quad 1$

\#\# $8 \quad 03 \quad 1$

\#\# $90303 \quad 1$

\#\# $10 \quad 03 \quad 1$

\#\# \# ... with 294 more rows

- multiple logical conditions: case_when

\# code driving \& non-driving based on travel modes (TRPTRANS column) data dictionary: http:/ /nhts.ornl.gov/tables09/CodebookPage.aspx?id=1084 use case_when dd $\%>\%$ mutate(driving=case_when(

TRPTRANS \% in\% c("01", "02", "03", "04", "05", "06", "07") 1, TRPTRANS \% in \% c("-1", "-7", "-8", "-9") as.double(NA), \# retain missing values as NA TRUE 0)) \%>\% select(TRPTRANS, driving)

\#\# \# A tibble: $304 \times 2$

\#\# TRPTRANS driving

\#\# <chr $><$ dbl $>$

\#\# $1 \quad 03 \quad 1$

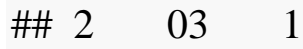

\#\# $3 \quad 03 \quad 1$

\#\# $4 \quad 03 \quad 1$

\#\# $5 \quad 03 \quad 1$

\#\# $6 \quad 03 \quad 1$

\#\# $7 \quad 03 \quad 1$

\#\# 8 103 1

\#\# $90303 \quad 1$

\#\# $10 \quad 03 \quad 1$

\#\# \# ... with 294 more rows 


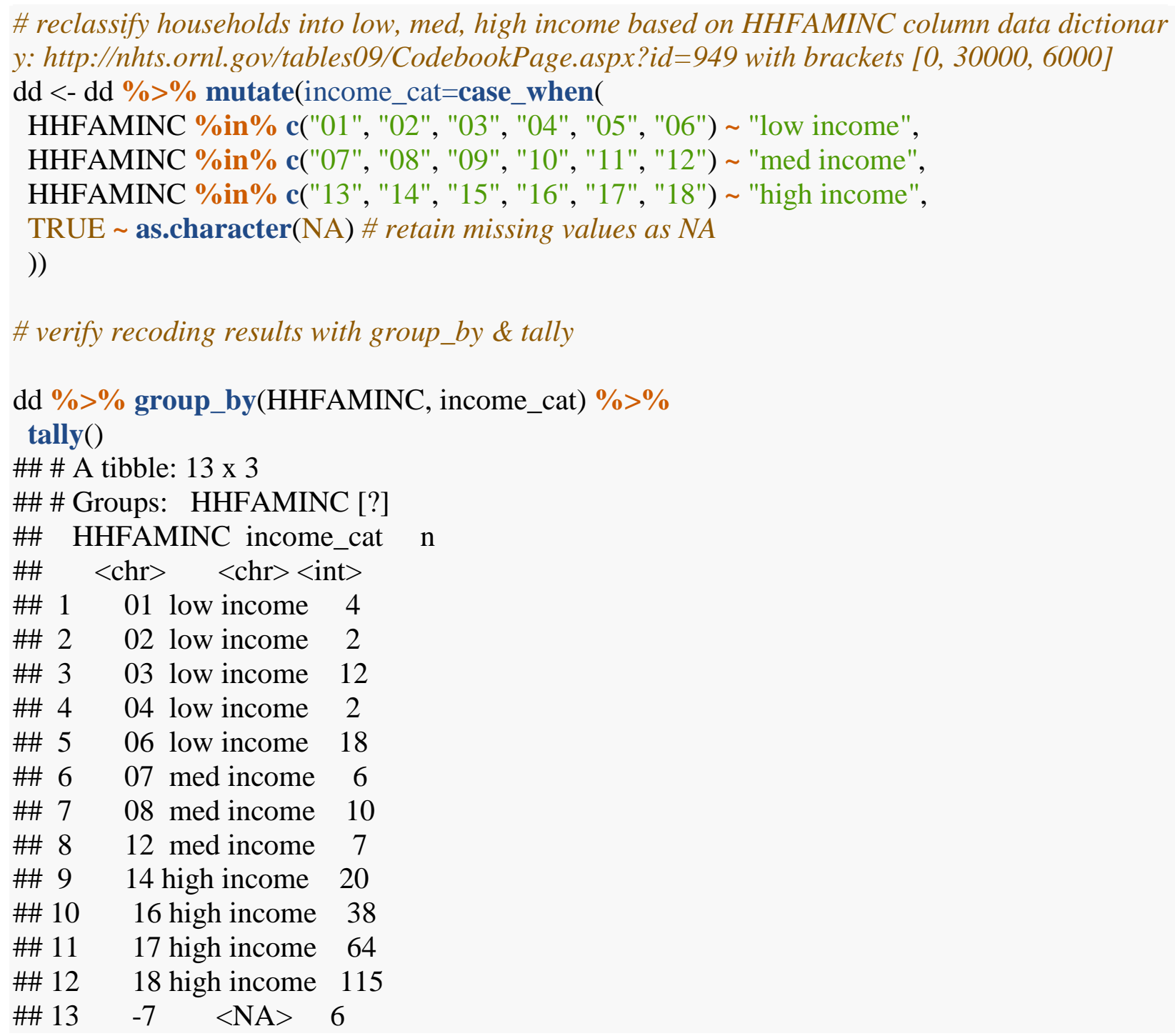

\subsubsection{Programming With dplyr}

- $\quad$ Non-standard Evaluation in dplyr 0.6+

- Programming with dplyr

\subsection{EXERCISE}

1. Filter days where there are missing values in bike counts and weather information. Count the number of days with missing values on either bike counts or weather information.

2. Calculate weekly, monthly, and annual bike counts from the daily bike counts data using dplyr verbs.

3. Join the bike counts data with the weather data. Which type of joins work best here?

4. With the NHTS2009 travel diaries data, how do you calculate total miles traveled (using any modes) and miles traveled by driving for each household (hint: the TRPMILES column contains information about trip distance for each member of a household). 
5. [Challenge] How do you compute the average household-level miles driving per capita by income categories (low, med, high)?

\subsection{LEARNING MORE}

1. Dataframe Manipulation with dplyr

2. Data Wrangling Cheatsheet

3. Hands-on dplyr tutorial for faster data manipulation in R by Data School, which includes a link to an R Markdown document and links to videos. 


\subsection{DATA VISUALIZATION WITH GGPLOT2}

\subsection{READINGS}

1. R4DS Chapter 3 Data Visualization

2. R4DS Chapter 7 Exploratory Data Analysis

\subsection{OVERVIEW}

ggplot2 is a grammar of graphics, a coherent system for describing and building graphs.

- data (data frame): ggplot(dataset)

- $\quad$ aesthetic mapping (coordination system, color, size, line type, position etc): aes()

- geometries: geom_point, geom_line, geom_bar, ...

- $\quad$ stat (summarization/transformation of data): stat_smooth,

- $\quad$ scale (map data values into computer values): scale_y_continuous(), scale_x_log10,...

- facet: facet_wrap, facet_grid and

- $\quad$ fine tuning coordinatae, theme, labels: coordinate_*, theme_bw, labs $(\mathrm{x}=\ldots, \mathrm{y}=\ldots)$

\subsection{TIPS}

- $\quad$ Keep your data tidy (three rules);

- $\quad$ Reshape your data (tall and thin often better for visualization and analysis); and

- Use factors (for reorder and better labeling).

\subsection{LESSON}

Lesson below is adapted from R4DS Chapter 3 Data Visualization.

\subsubsection{Prerequisites}

This session focuses on ggplot2, one of the core members of the tidyverse. To access the datasets, help pages, and functions that we will use in this chapter, load the tidyverse by running this code:

library(tidyverse)

library(ggplot2)

\subsubsection{First Steps}

Let's use our first graph to answer a question: Do cars with big engines use more fuel than cars with small engines? You probably already have an answer, but try to make your answer precise. What does the relationship between engine size and fuel efficiency look like? Is it positive? Negative? Linear? Nonlinear? 


\subsubsection{The mpg Data Frame}

You can test your answer with the mpg data frame found in ggplot2 (aka ggplot2::mpg). A data frame is a rectangular collection of variables (in the columns) and observations (in the rows). mpg contains observations collected by the U.S. Environment Protection Agency on 38 models of cars.

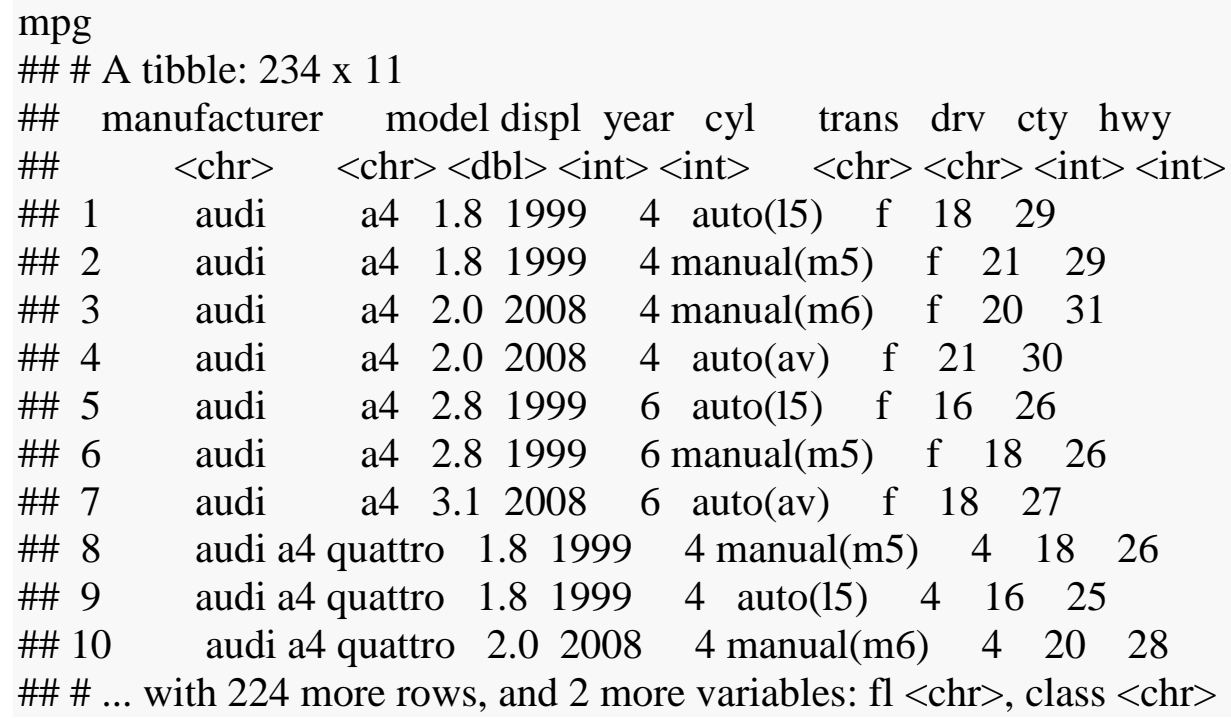

Among the variables in mpg are:

1. displ, a car's engine size, in liters.

2. hwy, a car's fuel efficiency on the highway, in miles per gallon (mpg). A car with low fuel efficiency consumes more fuel than a car with high fuel efficiency when they travel the same distance.

To learn more about mpg, open its help page by running ?mpg.

\subsubsection{Creating a ggplot}

To plot mpg, run this code to put displ on the x-axis and hwy on the $\mathrm{y}$-axis:

$\operatorname{ggplot}($ data $=\operatorname{mpg})+$

geom_point(mapping $=\operatorname{aes}(\mathrm{x}=\operatorname{displ}, \mathrm{y}=\mathrm{hwy})$ ) 


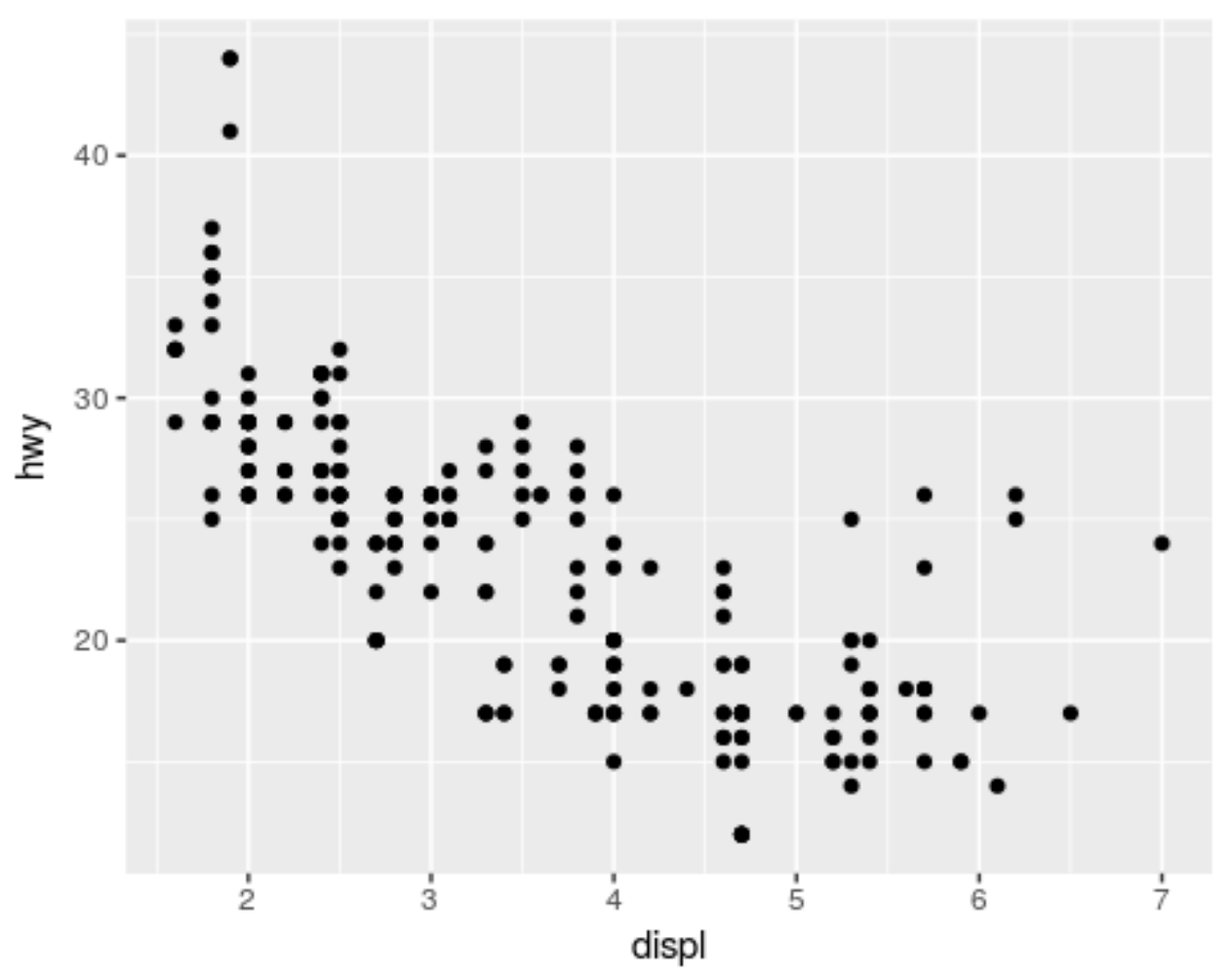

Figure 11.1 Scatter plot of highway mpg by displ

The plot shows a negative relationship between engine size (displ) and fuel efficiency (hwy). In other words, cars with big engines use more fuel. Does this confirm or refute your hypothesis about fuel efficiency and engine size?

With ggplot2, you begin a plot with the function ggplot(). ggplot() creates a coordinate system that you can add layers to. The first argument of ggplot() is the dataset to use in the graph. So ggplot $($ data $=\mathrm{mpg})$ creates an empty graph, but it's not very interesting so I'm not going to show it here.

You complete your graph by adding one or more layers to ggplot(). The function geom_point() adds a layer of points to your plot, which creates a scatter plot. ggplot2 comes with many geom functions that each add a different type of layer to a plot. You'll learn a whole bunch of them throughout this chapter.

Each geom function in ggplot2 takes a mapping argument. This defines how variables in your dataset are mapped to visual properties. The mapping argument is always paired with aes(), and the $\mathrm{x}$ and $\mathrm{y}$ arguments of aes() specify which variables to map to the $\mathrm{x}$ - and $\mathrm{y}$-axes. ggplot2 looks for the mapped variable in the data argument, in this case, mpg. 


\subsubsection{A Graphing Template}

Let's turn this code into a reusable template for making graphs with ggplot2. To make a graph, replace the bracketed sections in the code below with a dataset, a geom function, or a collection of mappings.

$\operatorname{ggplot}($ data $=<$ DATA $>)+$ $<$ GEOM_FUNCTION $>($ mapping $=$ aes $(<$ MAPPINGS $>))$

The rest of this session will show you how to complete and extend this template to make different types of graphs. We will begin with the $<$ MAPPINGS $>$ component.

\subsubsection{Aesthetic Mappings}

"The greatest value of a picture is when it forces us to notice what we never expected to see." — John Tukey

In the plot below, one group of points (highlighted in red) seems to fall outside of the linear trend. These cars have a higher mileage than you might expect. How can you explain these cars?

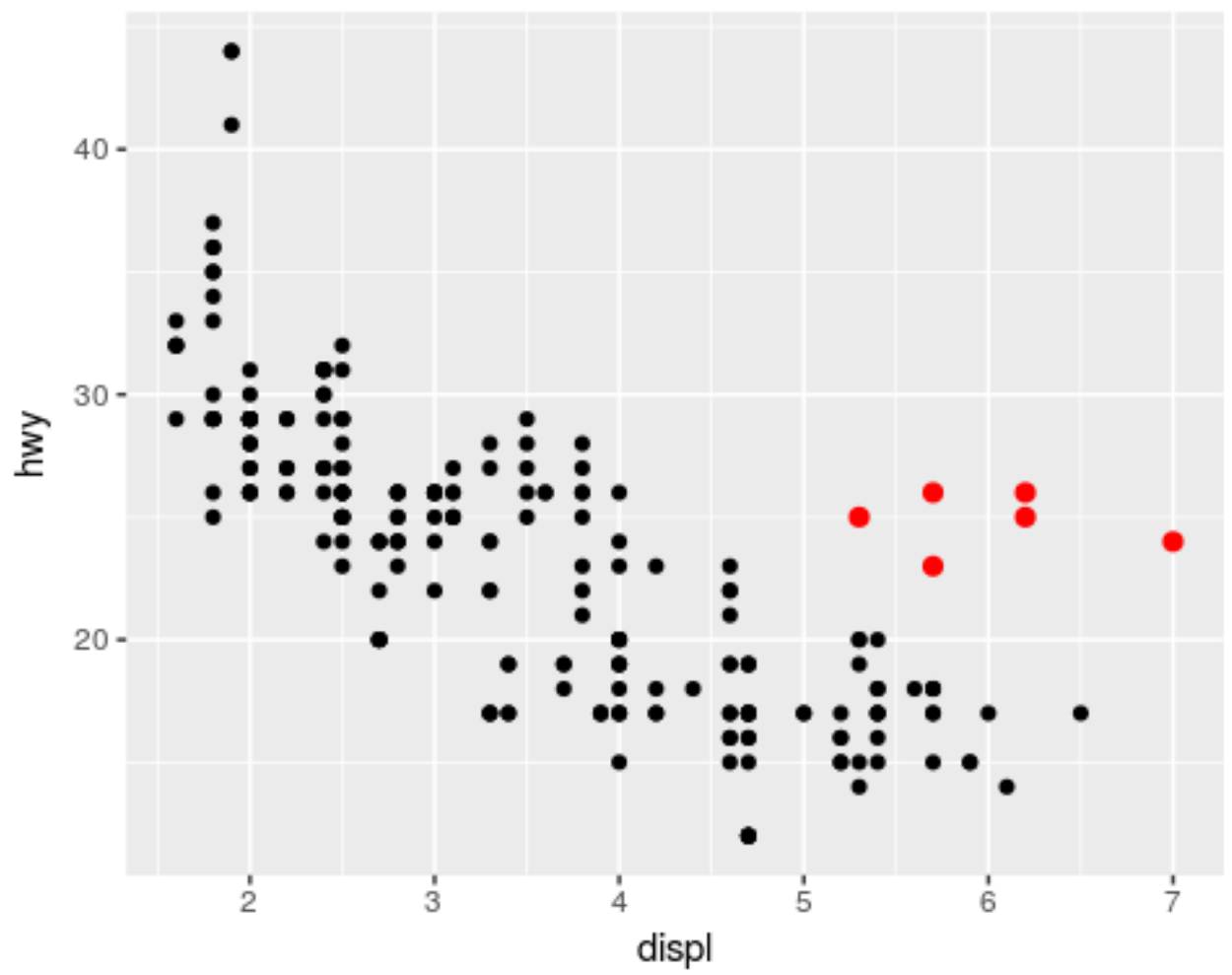

Figure 11.2 Scatter plot with some cars highlighted in red

Let's hypothesize that the cars are hybrids. One way to test this hypothesis is to look at the class value for each car. The class variable of the mpg dataset classifies cars into groups such as 
compact, midsize, and SUV. If the outlying points are hybrids, they should be classified as compact cars or, perhaps, subcompact cars (keep in mind that these data were collected before hybrid trucks and SUVs became popular).

You can add a third variable, like class, to a two-dimensional scatter plot by mapping it to an aesthetic. An aesthetic is a visual property of the objects in your plot. Aesthetics include things like the size, the shape, or the color of your points.

You can convey information about your data by mapping the aesthetics in your plot to the variables in your dataset. For example, you can map the colors of your points to the class variable to reveal the class of each car.

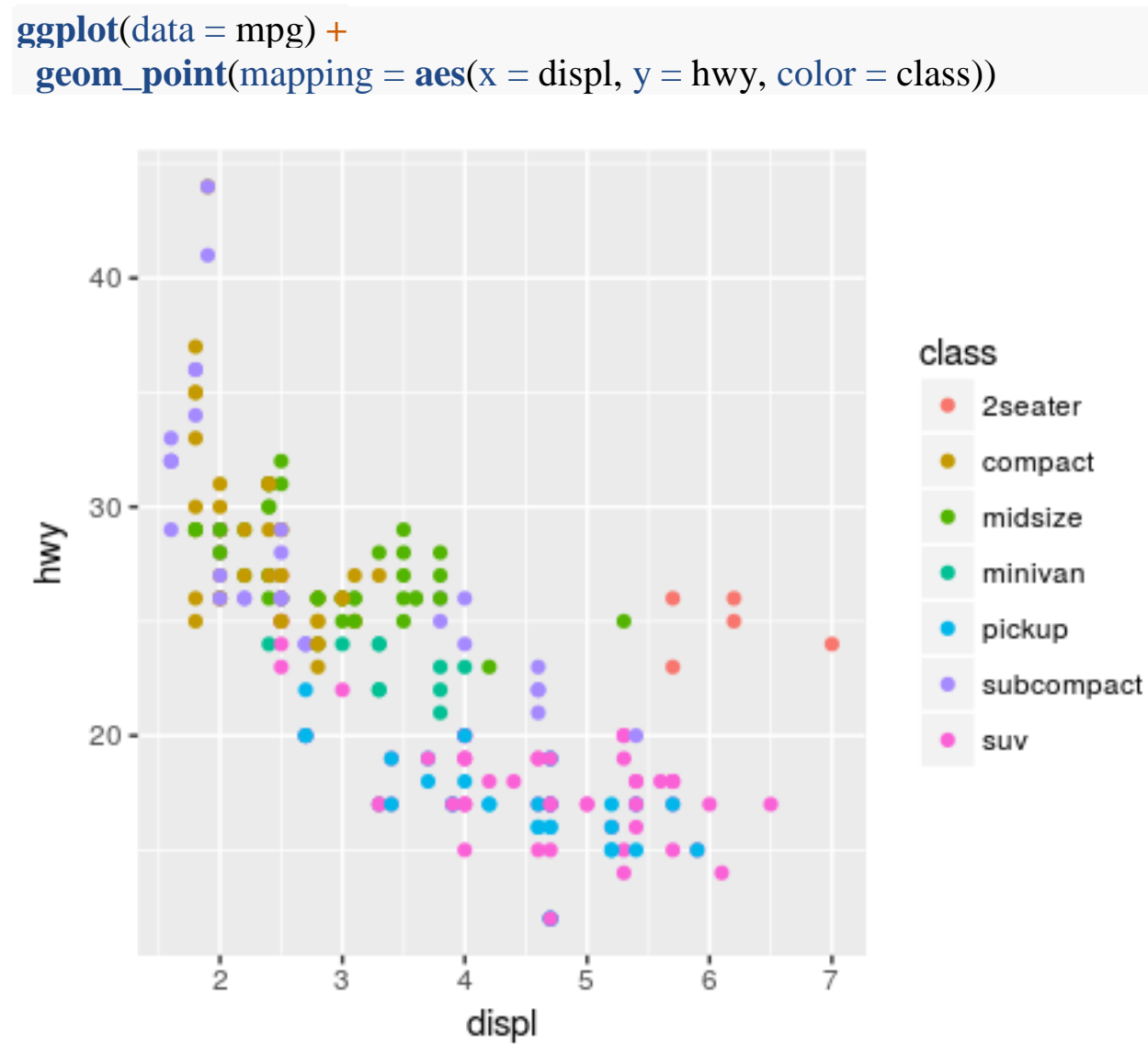

Figure 11.3 Scatter plot of highway mpg by displ, with class of cars differentiated using color

To map an aesthetic to a variable, associate the name of the aesthetic to the name of the variable inside aes(). ggplot2 will automatically assign a unique level of the aesthetic (here a unique color) to each unique value of the variable, a process known as scaling. ggplot2 will also add a legend that explains which levels correspond to which values.

The colors reveal that many of the unusual points are two-seater cars. These cars don't seem like hybrids, and are, in fact, sports cars! Sports cars have large engines like SUVs and pickup trucks, 
but small bodies like midsize and compact cars, which improves their gas mileage. In hindsight, these cars were unlikely to be hybrids since they have large engines.

In the above example, we mapped class to the color aesthetic, but we could have mapped class to the size aesthetic in the same way. In this case, the exact size of each point would reveal its class affiliation. We get a warning here because mapping an unordered variable (class) to an ordered aesthetic (size) is not a good idea.
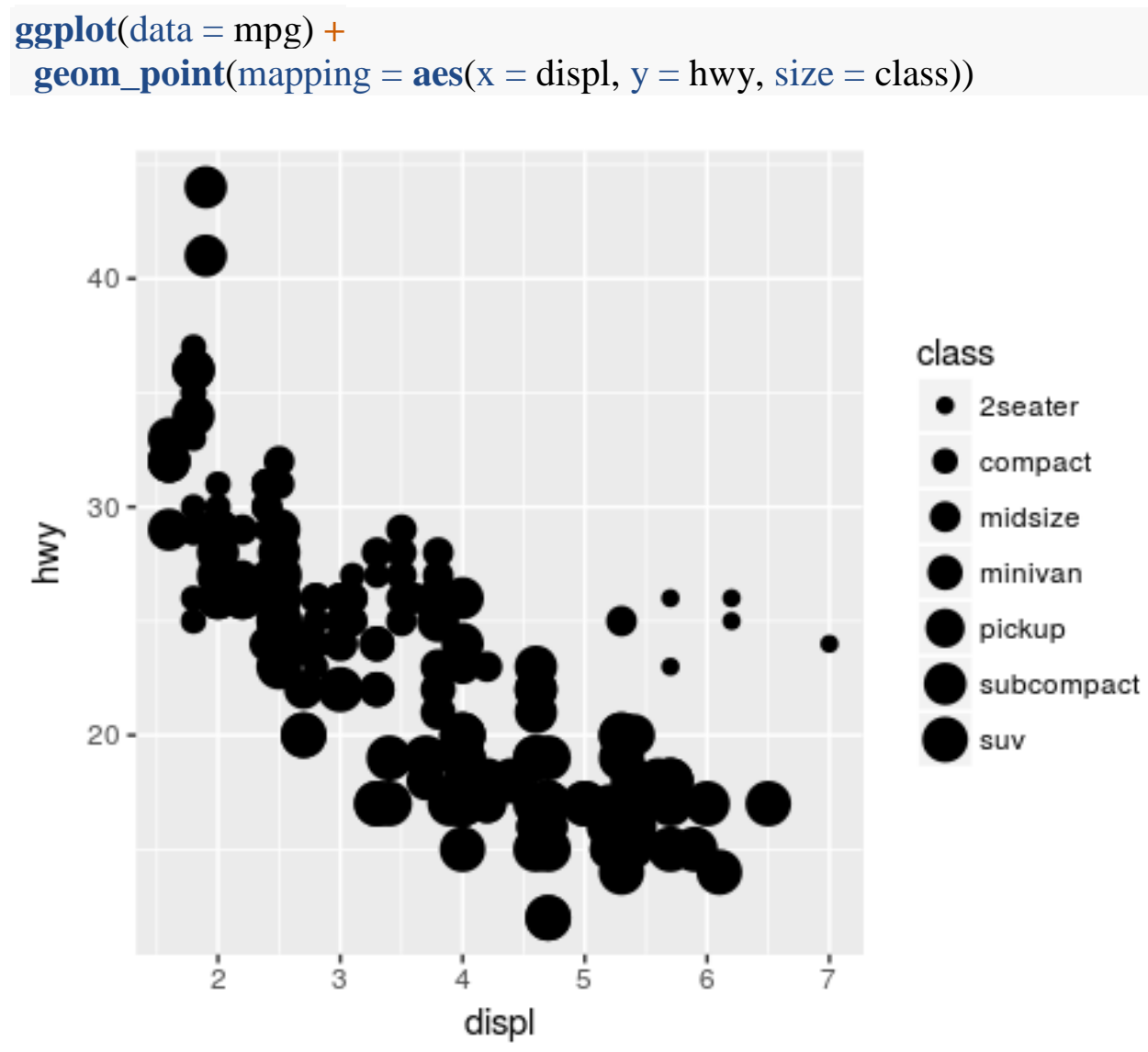

Figure 11.4 Scatter plot of highway mpg by displ, with class of cars differentiated using size

Or we could have mapped class to the alpha aesthetic, which controls the transparency of the points, or the shape of the points.

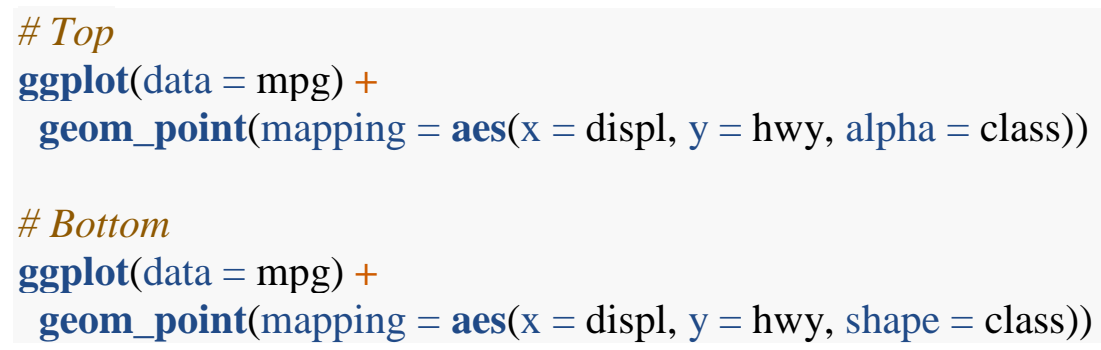




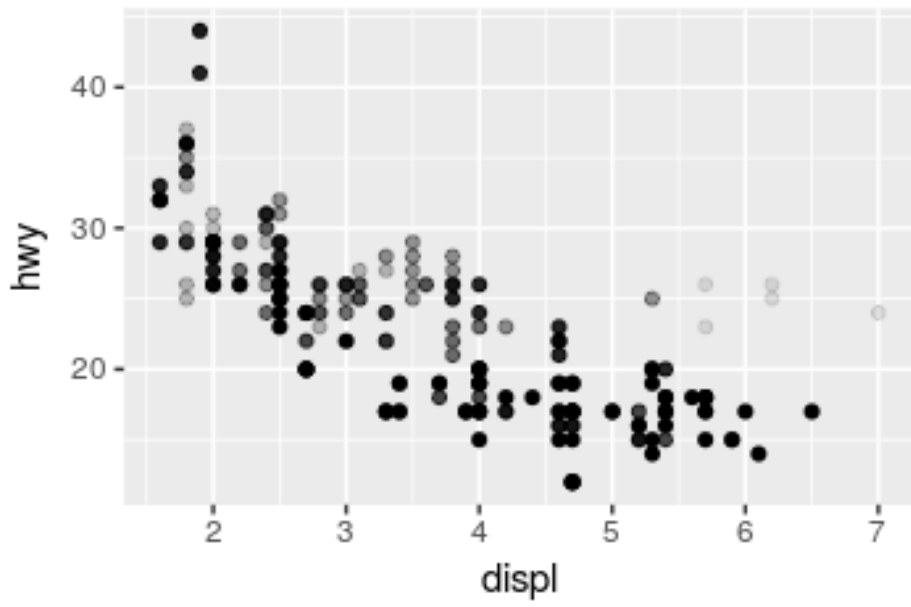

class

2seater

- compact

- midsize

- minivan

- pickup

- subcompact

- suv

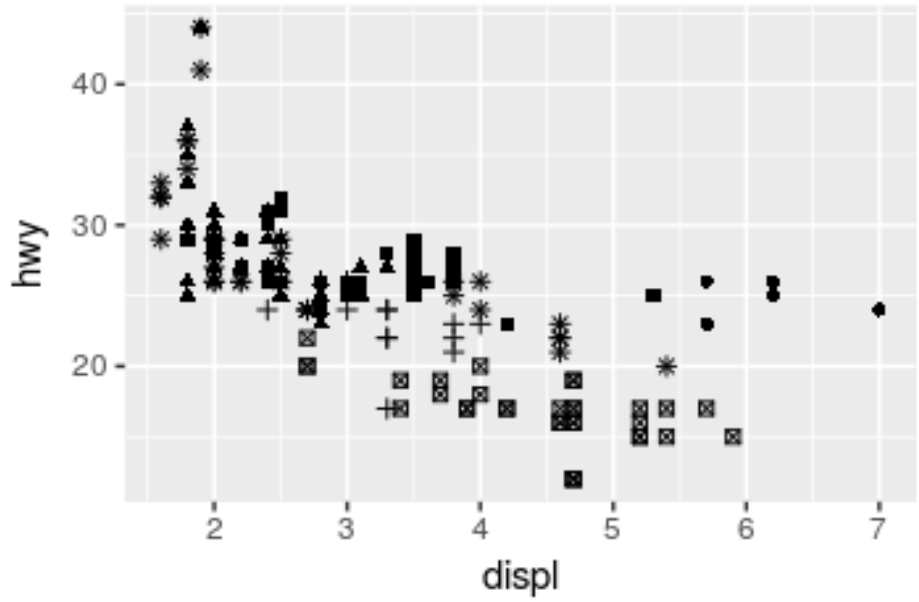

class

- 2seater

- compact

- midsize

+ minivan

pickup

* subcompact

suv

Figure 11.4 Scatter plot of highway mpg by displ, with class of cars differentiated using size

What happened to the SUVs? ggplot2 will only use six shapes at a time. By default, additional groups will go unplotted when you use the shape aesthetic.

For each aesthetic, you use aes() to associate the name of the aesthetic with a variable to display. The aes() function gathers together each of the aesthetic mappings used by a layer and passes them to the layer's mapping argument. The syntax highlights a useful insight about $\mathrm{x}$ and $\mathrm{y}$ : The $\mathrm{x}$ and $\mathrm{y}$ locations of a point are themselves aesthetics, visual properties that you can map to variables to display information about the data.

Once you map an aesthetic, ggplot2 takes care of the rest. It selects a reasonable scale to use with the aesthetic, and it constructs a legend that explains the mapping between levels and values. For $\mathrm{x}$ and $\mathrm{y}$ aesthetics, ggplot 2 does not create a legend, but it creates an axis line with tick marks and a label. The axis line acts as a legend; it explains the mapping between locations and values.

You can also set the aesthetic properties of your geom manually. For example, we can make all of the points in our plot blue: 


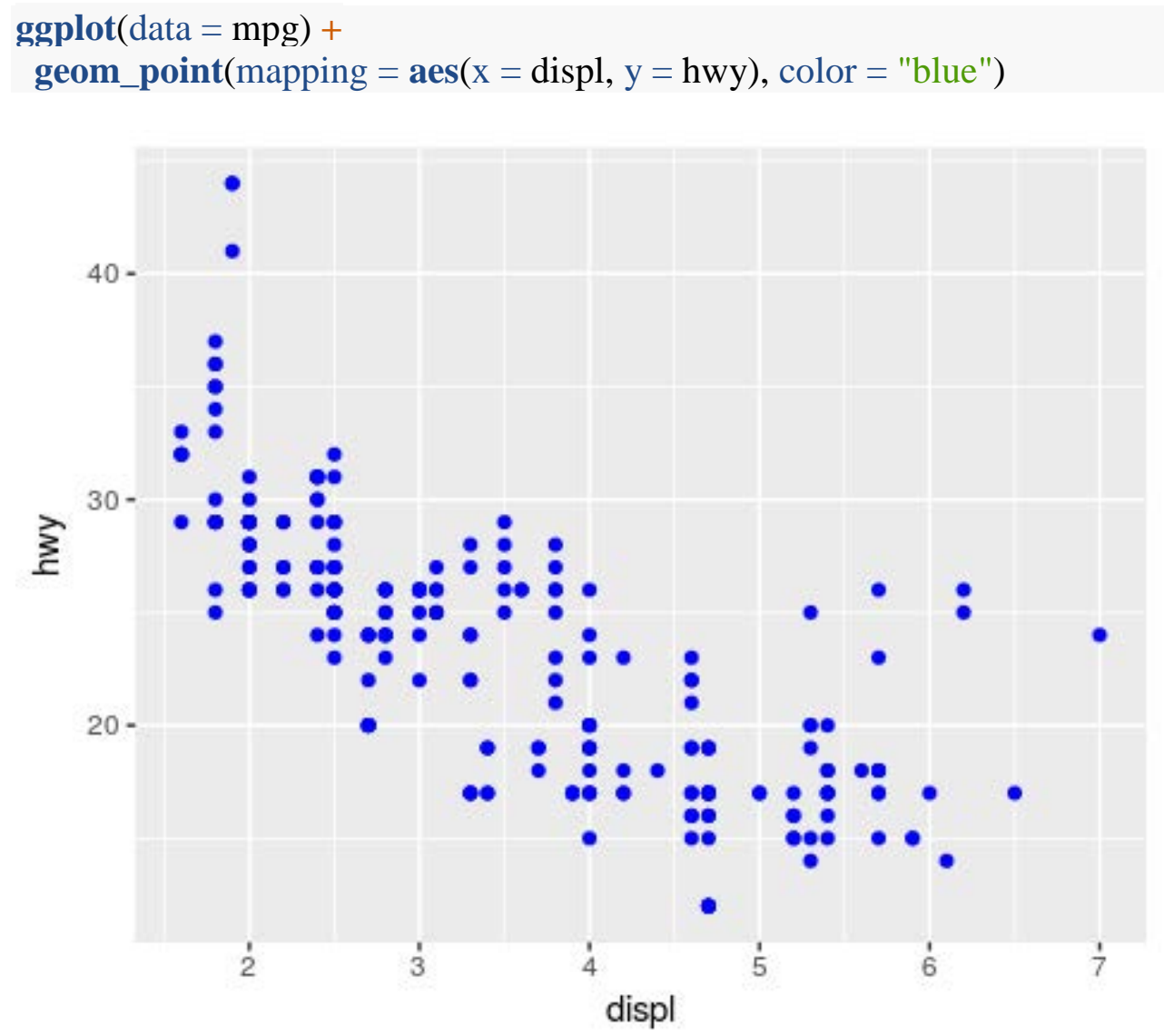

Figure 11.5 Scatter plot with color aesthetic manually set to blue

\subsubsection{Facets}

One way to add additional variables is with aesthetics. Another way, particularly useful for categorical variables, is to split your plot into facets, subplots that each display one subset of the data.

To facet your plot by a single variable, use facet_wrap(). The first argument of facet_wrap() should be a formula, which you create with $\sim$ followed by a variable name (here "formula" is the name of a data structure in $\mathrm{R}$, not a synonym for "equation"). The variable that you pass to facet_wrap() should be discrete.

$\operatorname{ggplot}($ data $=\mathrm{mpg})+$

geom_point(mapping $=$ aes $(\mathrm{x}=\operatorname{displ}, \mathrm{y}=\mathrm{hwy}))+$

facet_wrap $(\sim$ class, nrow $=2)$ 


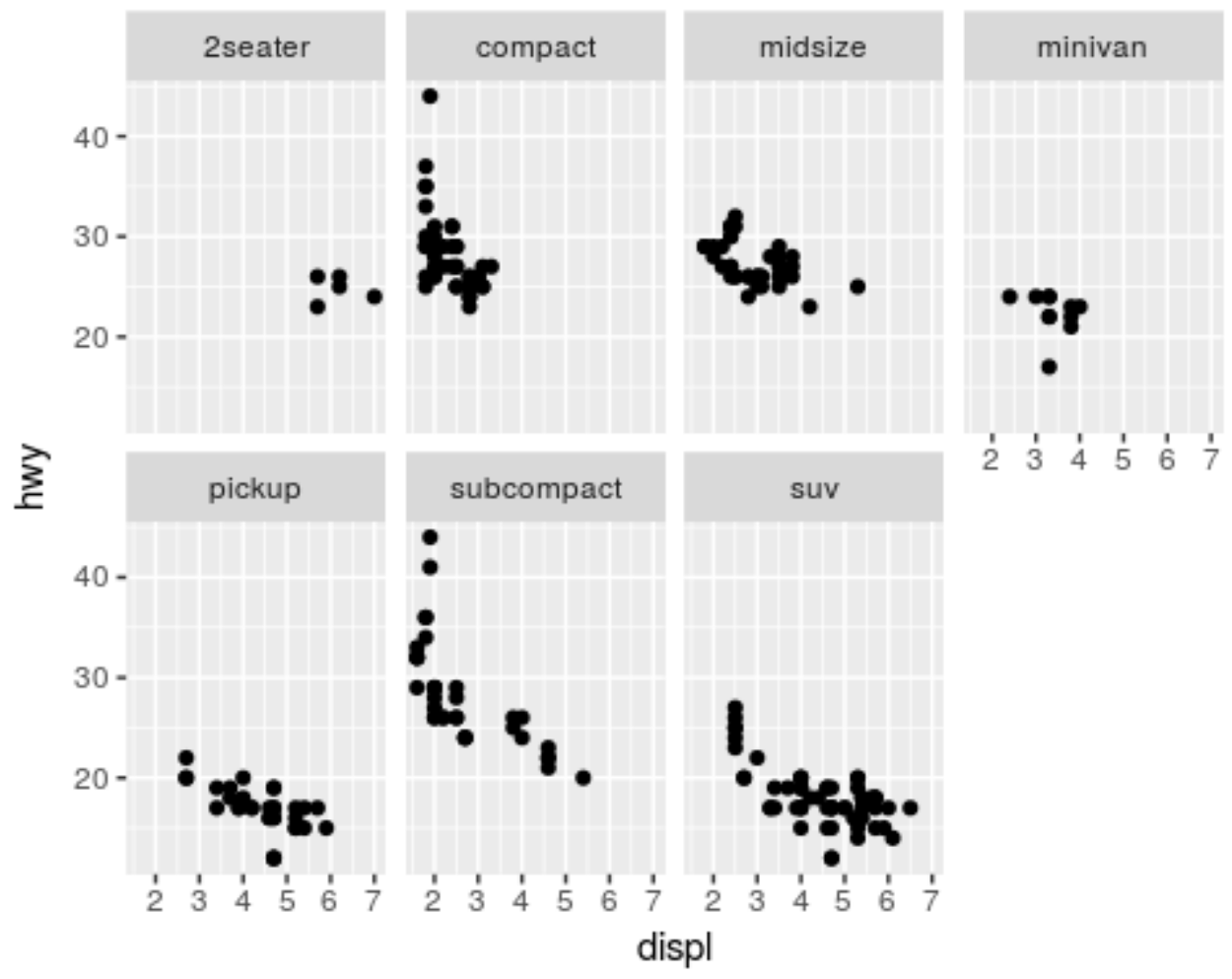

Figure 11.6 Scatter plots of highway mpg by displ, faceted by class

To facet your plot on the combination of two variables, add facet_grid() to your plot call. The first argument of facet_grid() is also a formula. This time, the formula should contain two variable names separated by a $\sim$

$\operatorname{ggplot}($ data $=\mathrm{mpg})+$

geom_point(mapping $=\operatorname{aes}(\mathrm{x}=\operatorname{displ}, \mathrm{y}=\mathrm{hwy}))+$

facet_grid(drv cyl) 


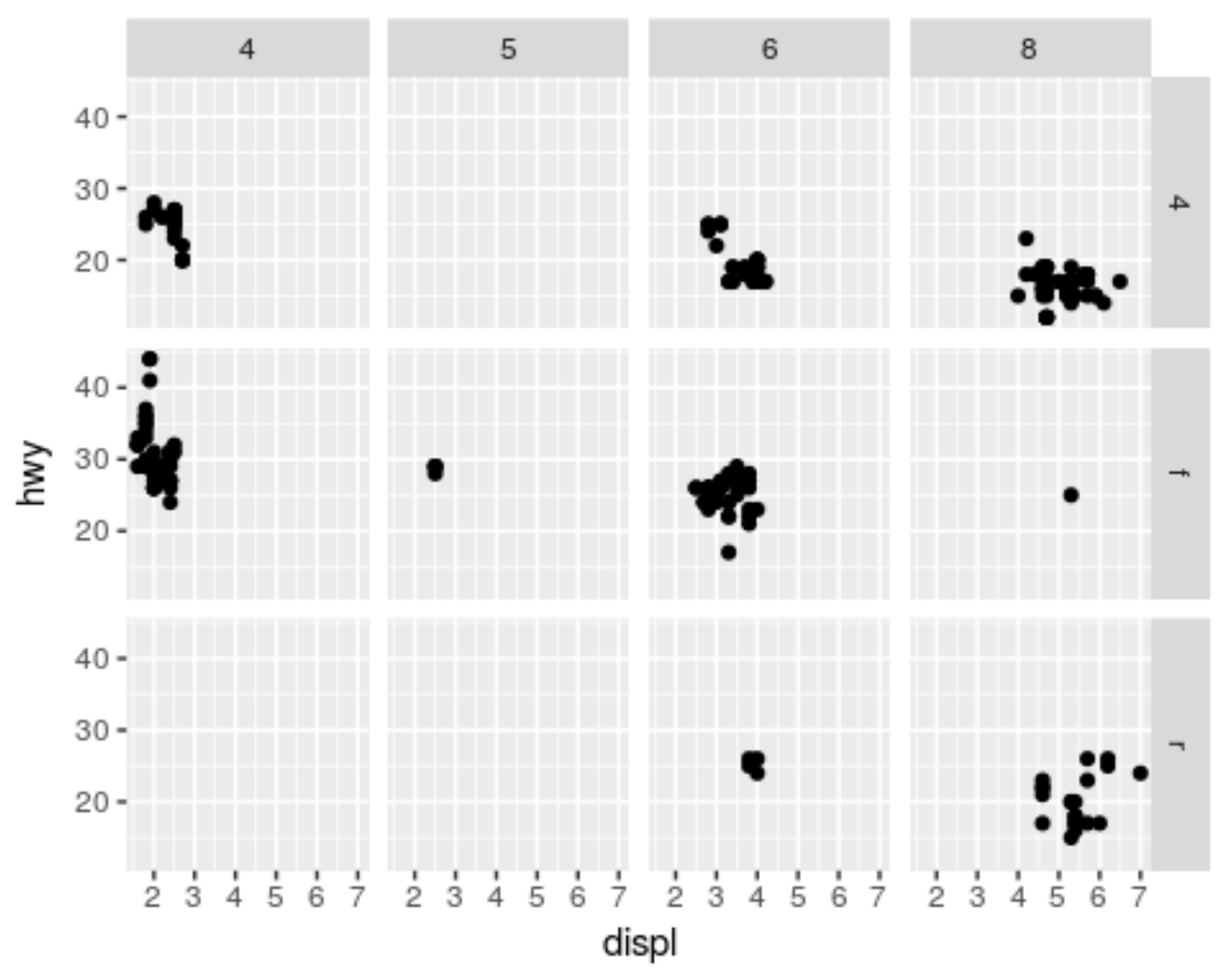

Figure 11.6 Scatter plots of highway mpg by displ, faceted by class

If you prefer to not facet in the rows or columns dimension, use a . instead of a variable name (e.g., + facet_grid(. cyl).

\subsubsection{Geometric Objects}

How are these two plots similar? 

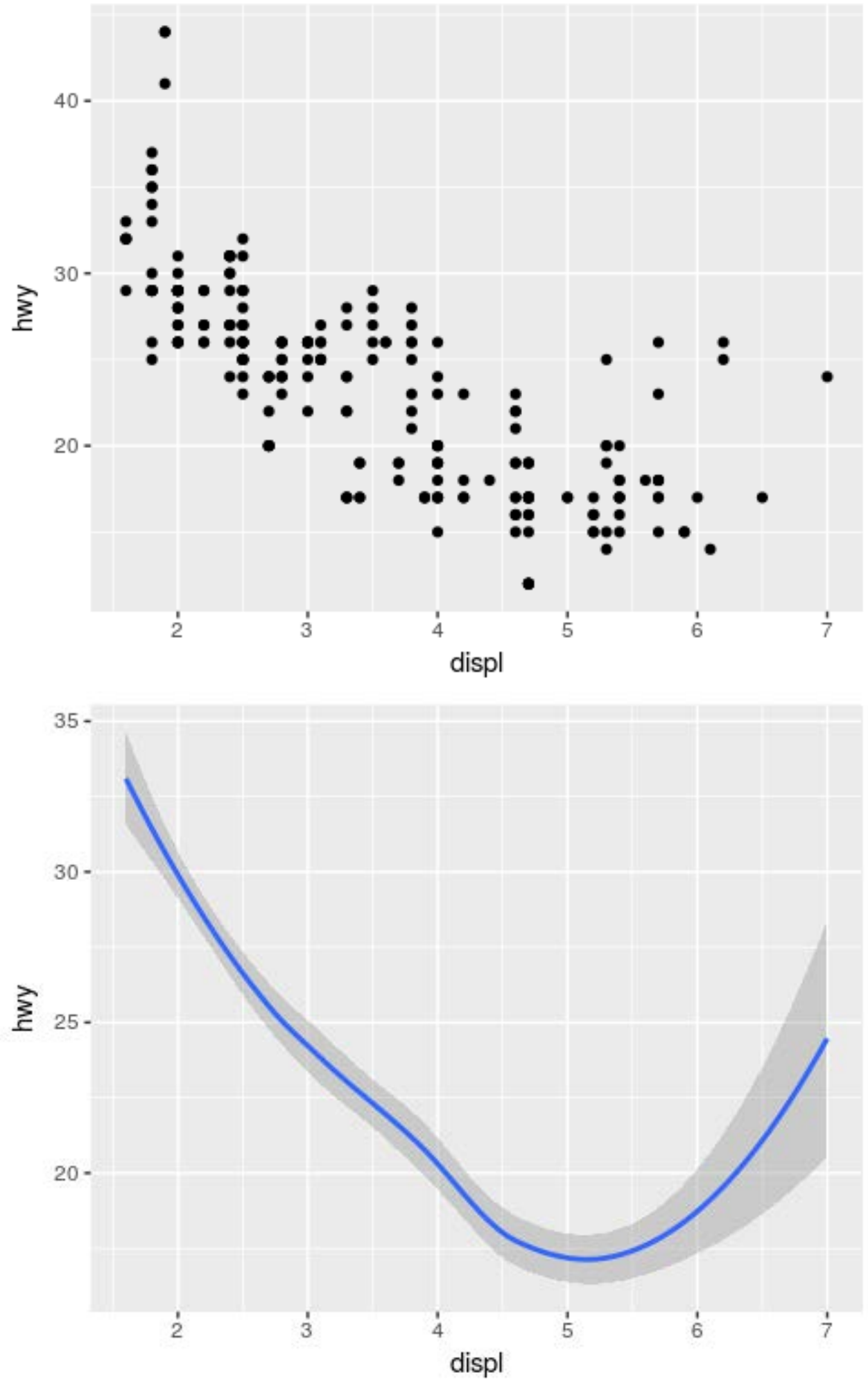

Figure 11.7 Scatter plot and smooth curve of highway mpg by displ 
Both plots contain the same $\mathrm{x}$ and $\mathrm{y}$ variables, and both describe the same data. But the plots are not identical. Each uses a different visual object to represent the data. In ggplot2 syntax, we say that they use different geoms.

A geom is the geometrical object that a plot uses to represent data. People often describe plots by the type of geom that the plot uses. For example, bar charts use bar geoms, line charts use line geoms, boxplots use boxplot geoms, and so on. Scatter plots break the trend; they use the point geom. As we see above, you can use different geoms to plot the same data. The plot on the left uses the point geom, and the plot on the right uses the smooth geom, a smooth line fitted to the data.

To change the geom in your plot, change the geom function that you add to ggplot(). For instance, to make the plots above, you can use this code:

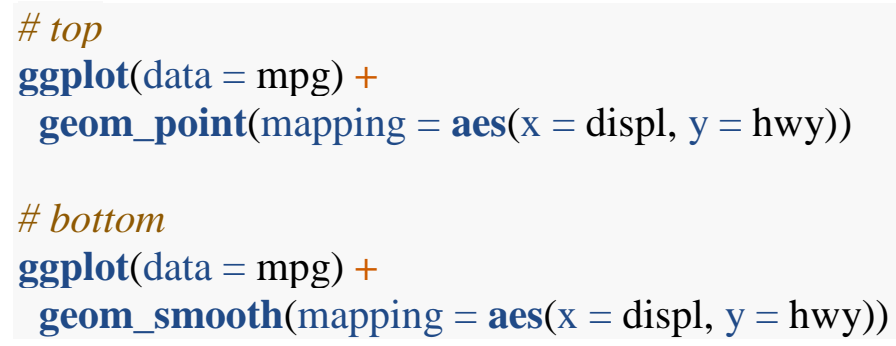

Every geom function in ggplot2 takes a mapping argument. However, not every aesthetic works with every geom. You could set the shape of a point, but you couldn't set the "shape" of a line. On the other hand, you could set the linetype of a line. geom_smooth() will draw a different line, with a different linetype, for each unique value of the variable that you map to linetype.

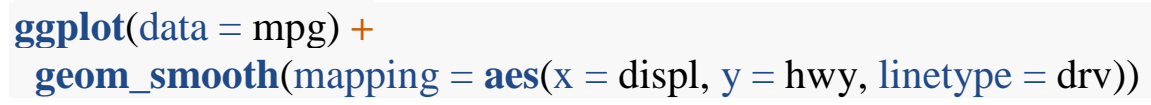




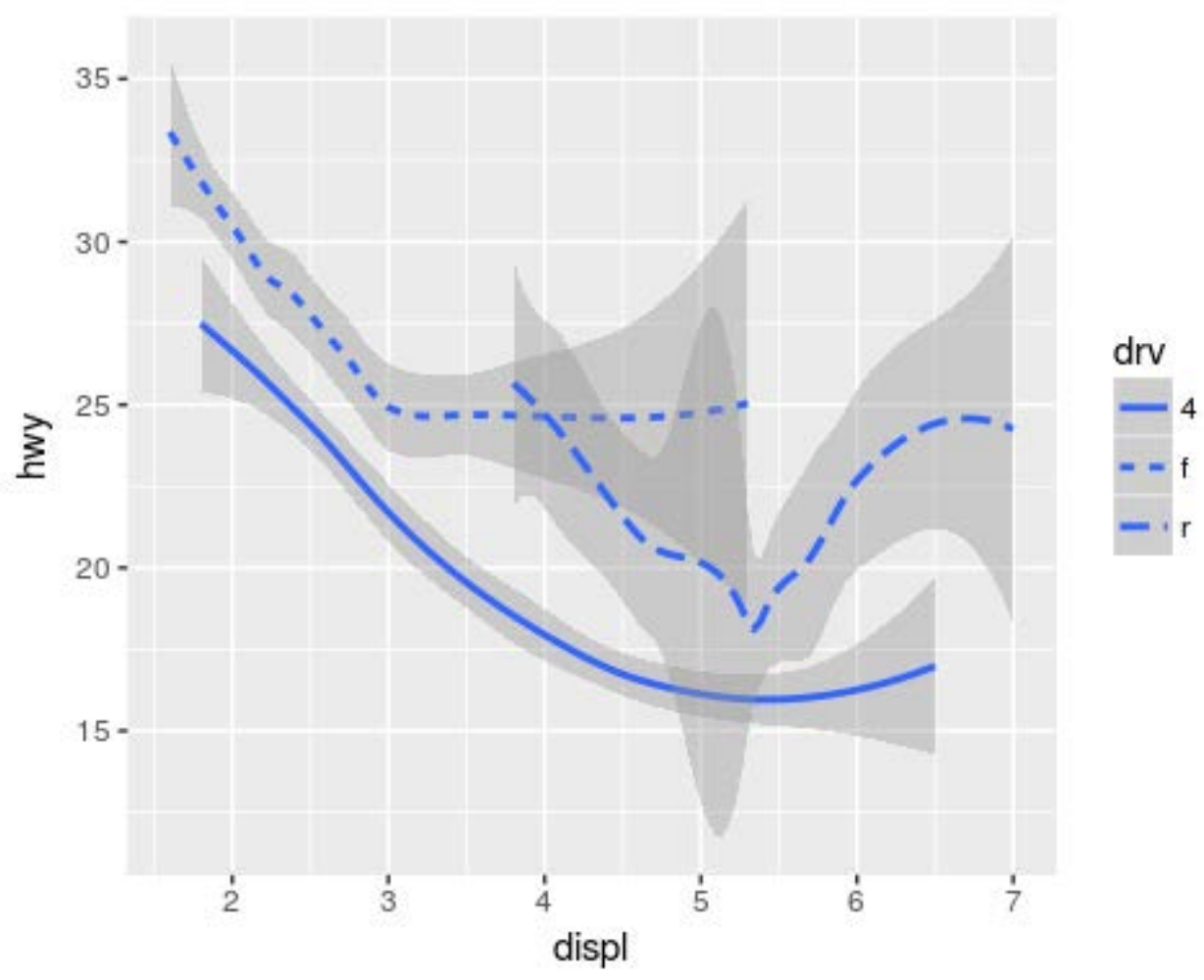

Figure 11.8 Smooth curves of highway mpg by displ, grouped by drv

Here geom_smooth() separates the cars into three lines based on their drv value, which describes a car's drivetrain. One line describes all of the points with a 4 value, one line describes all of the points with an $\mathrm{f}$ value, and one line describes all of the points with an $\mathrm{r}$ value. Here, 4 stands for four-wheel drive, $\mathrm{f}$ for front-wheel drive, and $\mathrm{r}$ for rear-wheel drive.

If this sounds strange, we can make it more clear by overlaying the lines on top of the raw data and then coloring everything according to drv. 


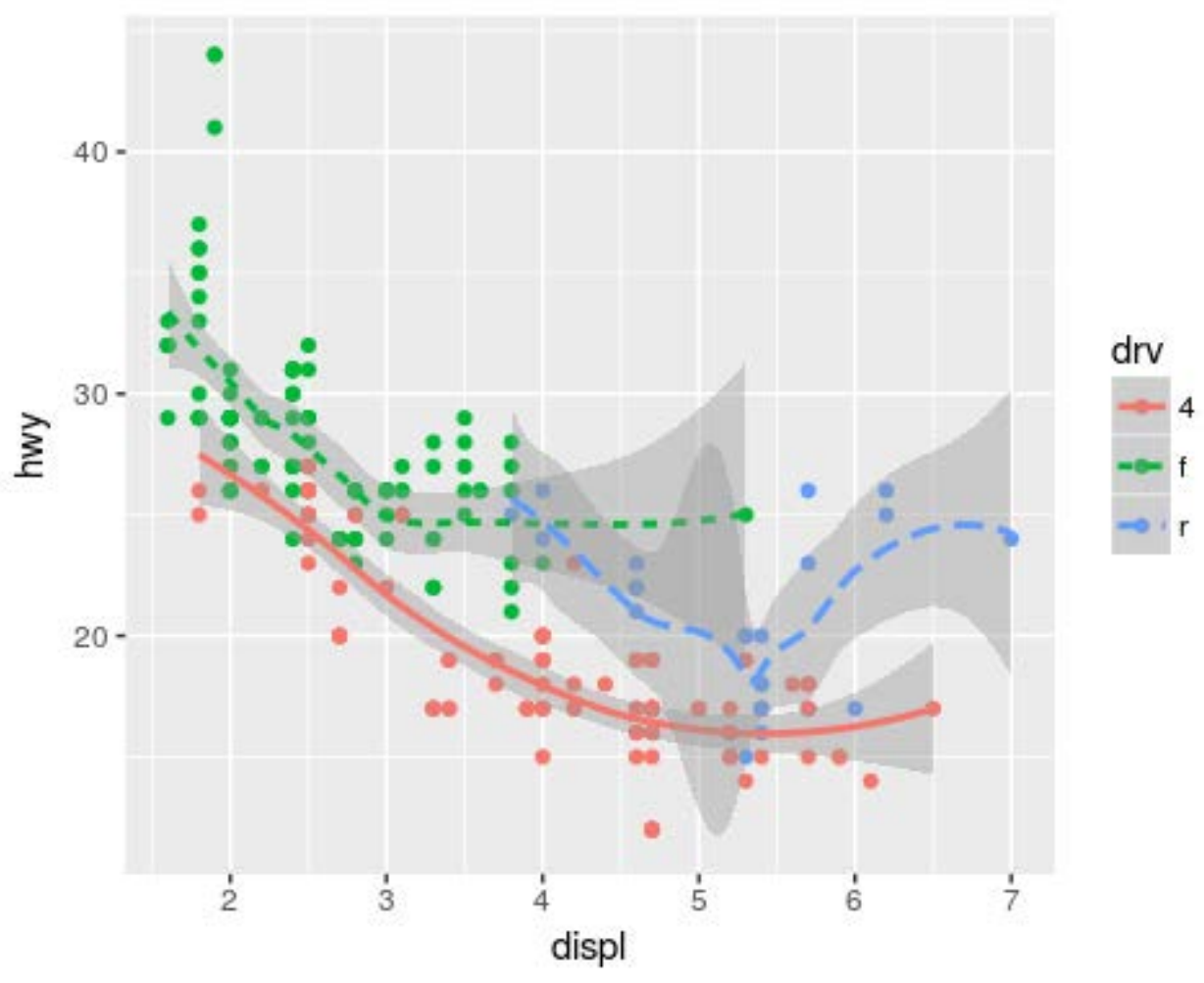

Figure 11.9 Scatter plot and smooth curves of highway mpg by displ, grouped by drv

Notice that this plot contains two geoms in the same graph! If this makes you excited, buckle up. In the next section, we will learn how to place multiple geoms in the same plot.

ggplot2 provides over 30 geoms, and extension packages provide even more (see https://www.ggplot2-exts.org for a sampling). The best way to get a comprehensive overview is the ggplot2 cheatsheet, which you can find at https://www.rstudio.com/resources/cheatsheets/. To learn more about any single geom, use help: ?geom_smooth.

Many geoms, like geom_smooth(), use a single geometric object to display multiple rows of data. For these geoms, you can set the group aesthetic to a categorical variable to draw multiple objects. ggplot2 will draw a separate object for each unique value of the grouping variable. In practice, ggplot2 will automatically group the data for these geoms whenever you map an aesthetic to a discrete variable (as in the linetype example). It is convenient to rely on this feature because the group aesthetic by itself does not add a legend or distinguishing features to the geoms.

$$
\begin{aligned}
& \text { ggplot }(\text { data }=\mathrm{mpg})+ \\
& \text { geom_smooth(mapping }=\operatorname{aes}(\mathrm{x}=\operatorname{displ}, \mathrm{y}=\mathrm{hwy})) \\
& \text { ggplot }(\text { data }=\mathrm{mpg})+ \\
& \text { geom_smooth(mapping }=\operatorname{aes}(\mathrm{x}=\operatorname{displ}, \mathrm{y}=\mathrm{hwy} \text {, group }=\mathrm{drv}))
\end{aligned}
$$



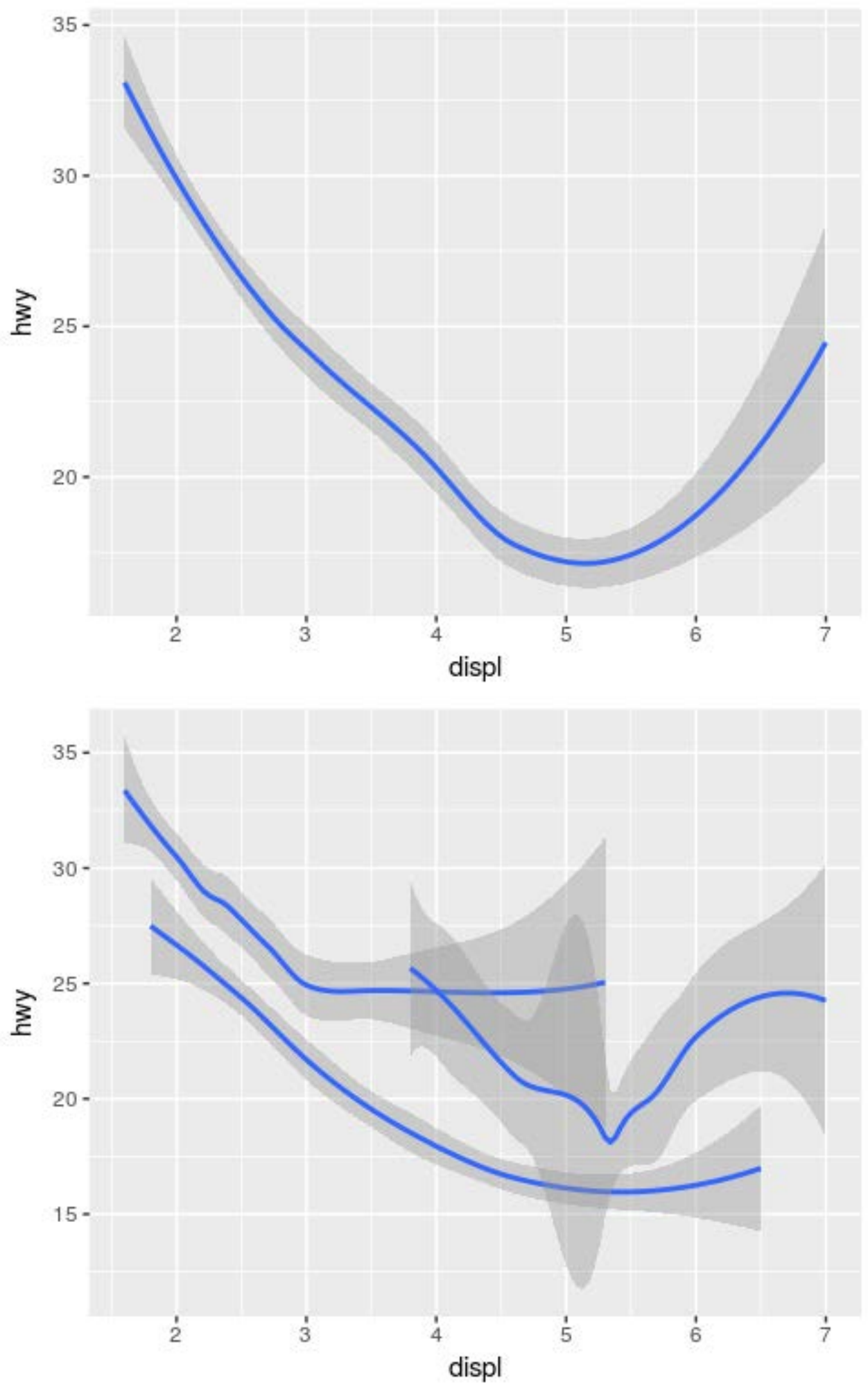

Figure 11.10 Smooth curves of highway mpg by displ 
To display multiple geoms in the same plot, add multiple geom functions to ggplot():
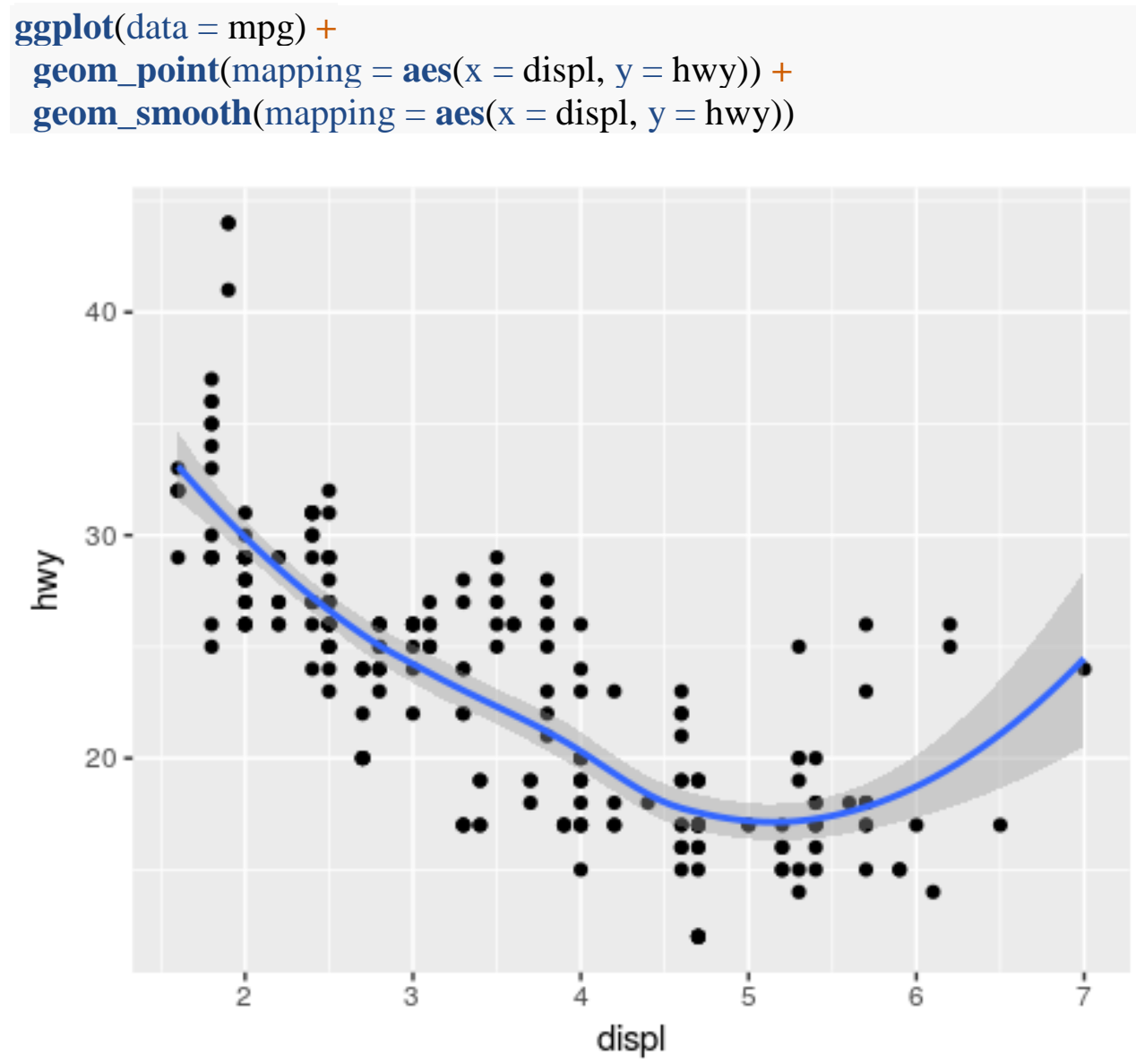

Figure 11.11 Scatter plot of highway mpg by displ super-imposed with stat_smooth

This, however, introduces some duplication in our code. Imagine if you wanted to change the yaxis to display cty instead of hwy. You'd need to change the variable in two places, and you might forget to update one. You can avoid this type of repetition by passing a set of mappings to ggplot(). ggplot2 will treat these mappings as global mappings that apply to each geom in the graph. In other words, this code will produce the same plot as the previous code:

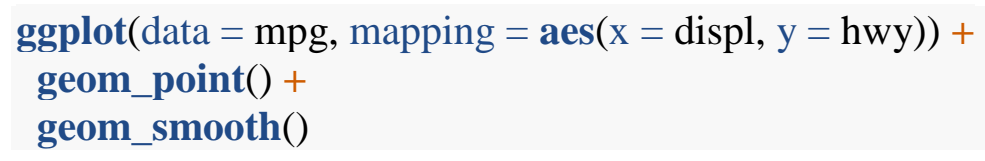

\subsubsection{Statistical Transformations}

Next, let's take a look at a bar chart. Bar charts seem simple, but they are interesting because they reveal something subtle about plots. Consider a basic bar chart, as drawn with geom_bar(). The following chart displays the total number of diamonds in the diamonds dataset, grouped by cut. The diamonds dataset comes in ggplot2 and contains information about 54,000 diamonds, 
including the price, carat, color, clarity, and cut of each diamond. The chart shows that more diamonds are available with high-quality cuts than with low-quality cuts.
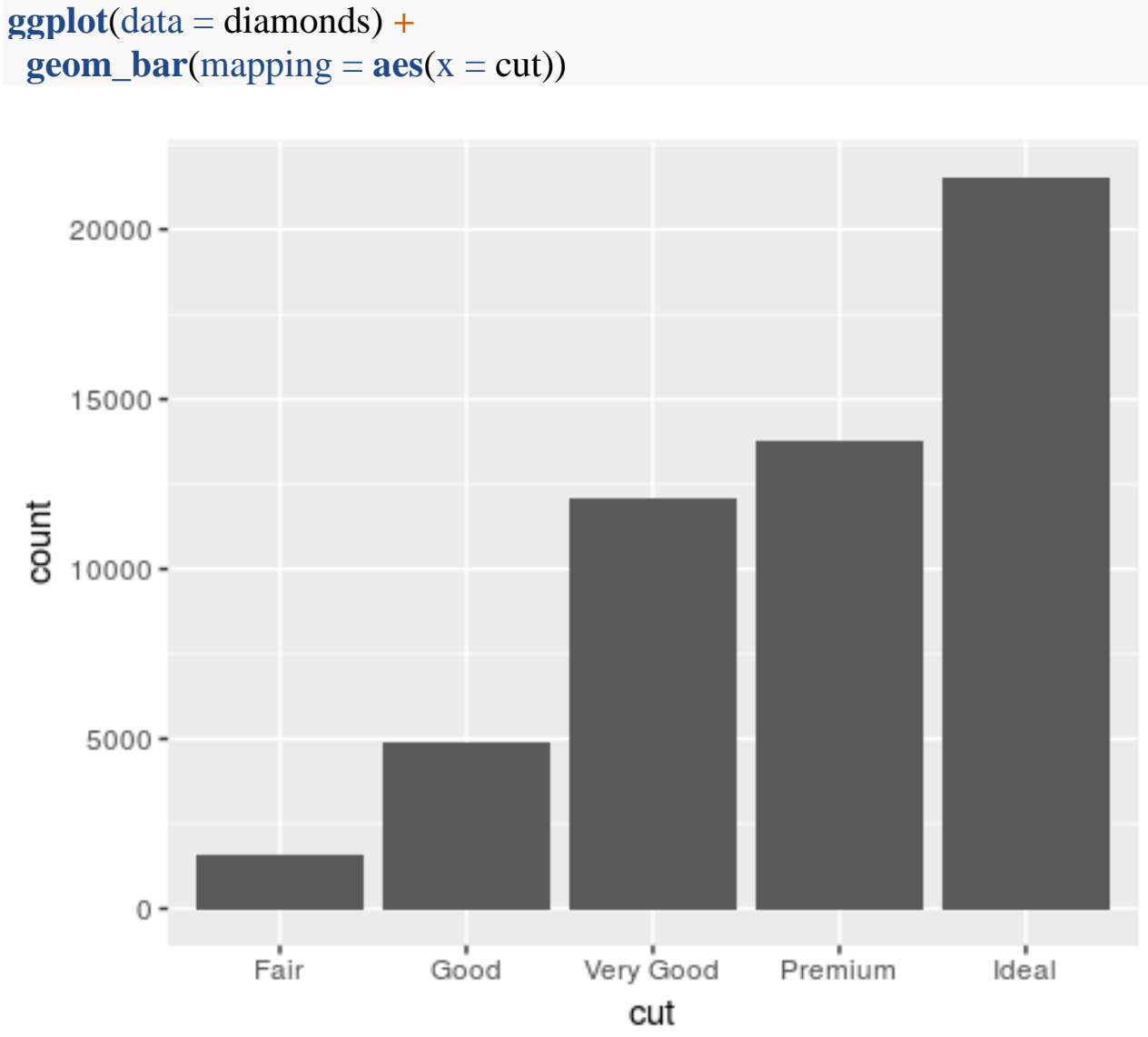

Figure 11.12 Bar chart of diamonds by cut

On the x-axis, the chart displays cut, a variable for diamonds. On the $\mathrm{y}$-axis, it displays count, but count is not a variable for diamonds! Where does count come from? Many graphs, like scatter plots, plot the raw values of your dataset. Other graphs, like bar charts, calculate new values to plot:

- Bar charts, histograms, and frequency polygons bin your data and then plot bin counts, the number of points that fall in each bin.

- $\quad$ Smoothers fit a model to your data and then plot predictions from the model.

- Boxplots compute a robust summary of the distribution and then display a specially formatted box.

The algorithm used to calculate new values for a graph is called a stat, short for statistical transformation. The figure below describes how this process works with geom_bar(). 


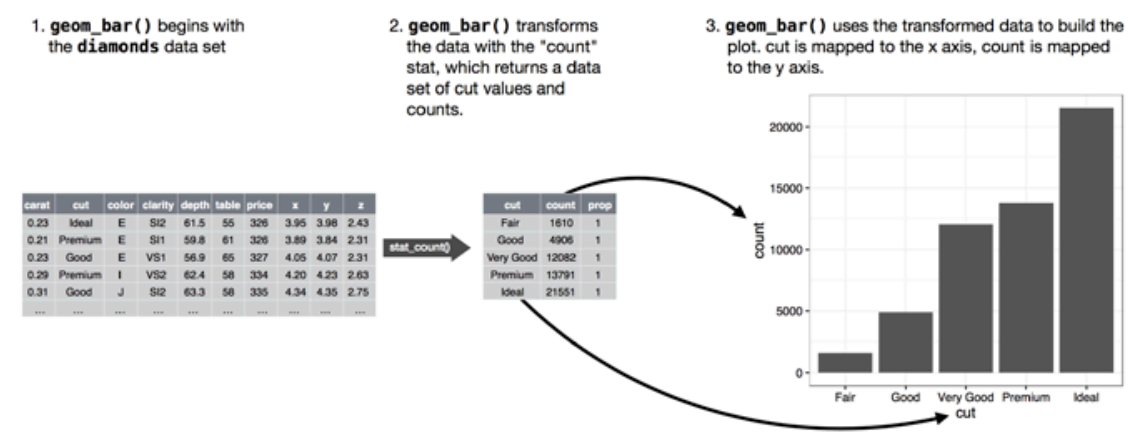

Figure 11.13 Process behind geom_bar

You can learn which stat a geom uses by inspecting the default value for the stat argument. For example, ?geom_bar shows the default value for stat is "count," which means that geom_bar() uses stat_count(). stat_count() is documented on the same page as geom_bar(), and if you scroll down you can find a section called "computed variables." That tells that it computes two new variables: count and prop.

You can generally use geoms and stats interchangeably. For example, you can recreate the previous plot using stat_count() instead of geom_bar():

ggplot $($ data $=$ diamonds $)+$

stat_count $($ mapping $=\operatorname{aes}(\mathrm{x}=$ cut $))$ 


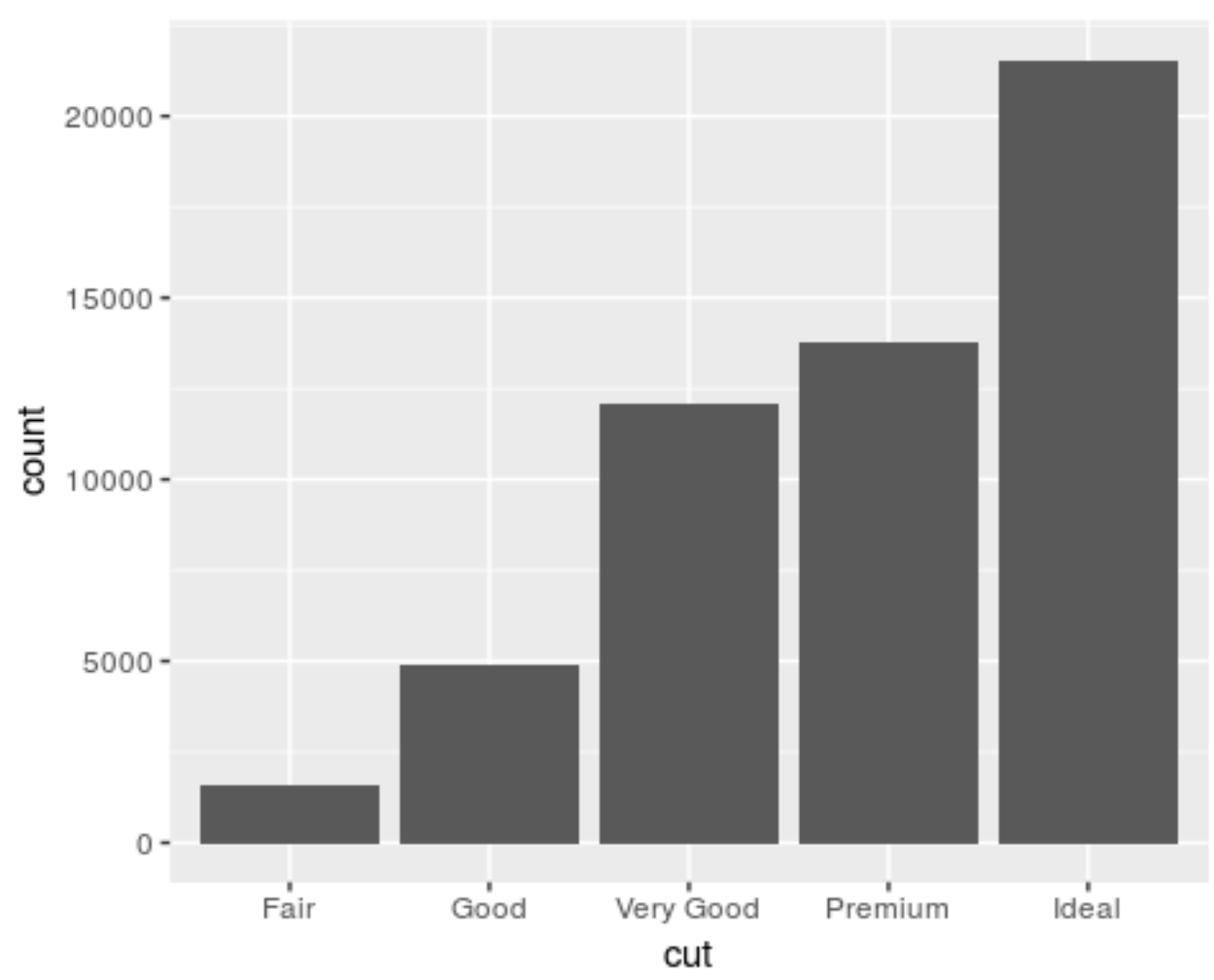

Figure 11.14 Bar chart of diamonds by cut created with stat_count

This works because every geom has a default stat, and every stat has a default geom. This means that you can typically use geoms without worrying about the underlying statistical transformation. There are three reasons you might need to use a stat explicitly:

1. You might want to override the default stat. In the code below, I change the stat of geom_bar() from count (the default) to identify. This lets me map the height of the bars to the raw values of a $y$ variable. Unfortunately, when people talk about bar charts casually, they might be referring to this type of bar chart, where the height of the bar is already present in the data, or the previous bar chart, where the height of the bar is generated by counting rows.

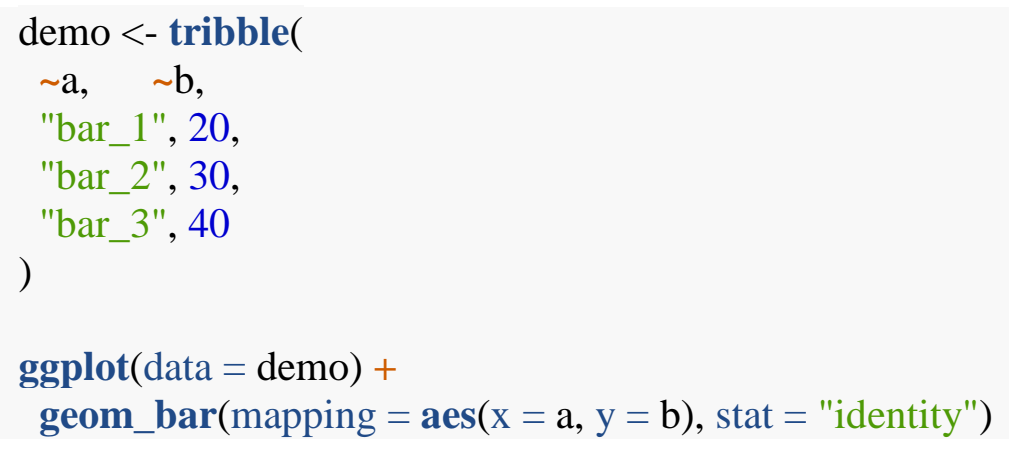




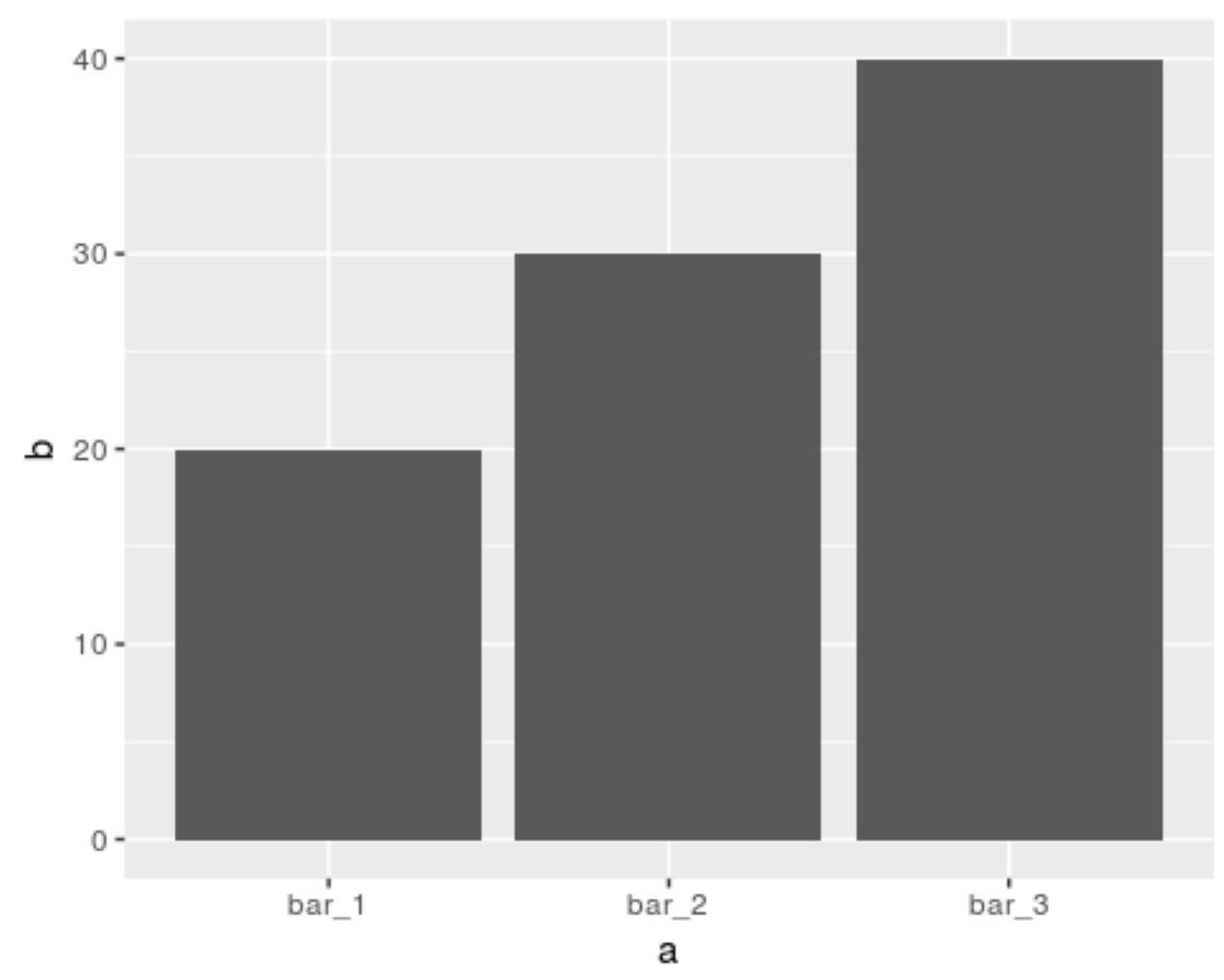

Figure 11.15 Bar chart created with stat=identity

2. You might want to override the default mapping from transformed variables to aesthetics. For example, you might want to display a bar chart of proportion, rather than count: ggplot $($ data $=$ diamonds $)+$ geom_bar(mapping $=$ aes $(\mathrm{x}=$ cut, $\mathrm{y}=$..prop.., group $=1))$ 


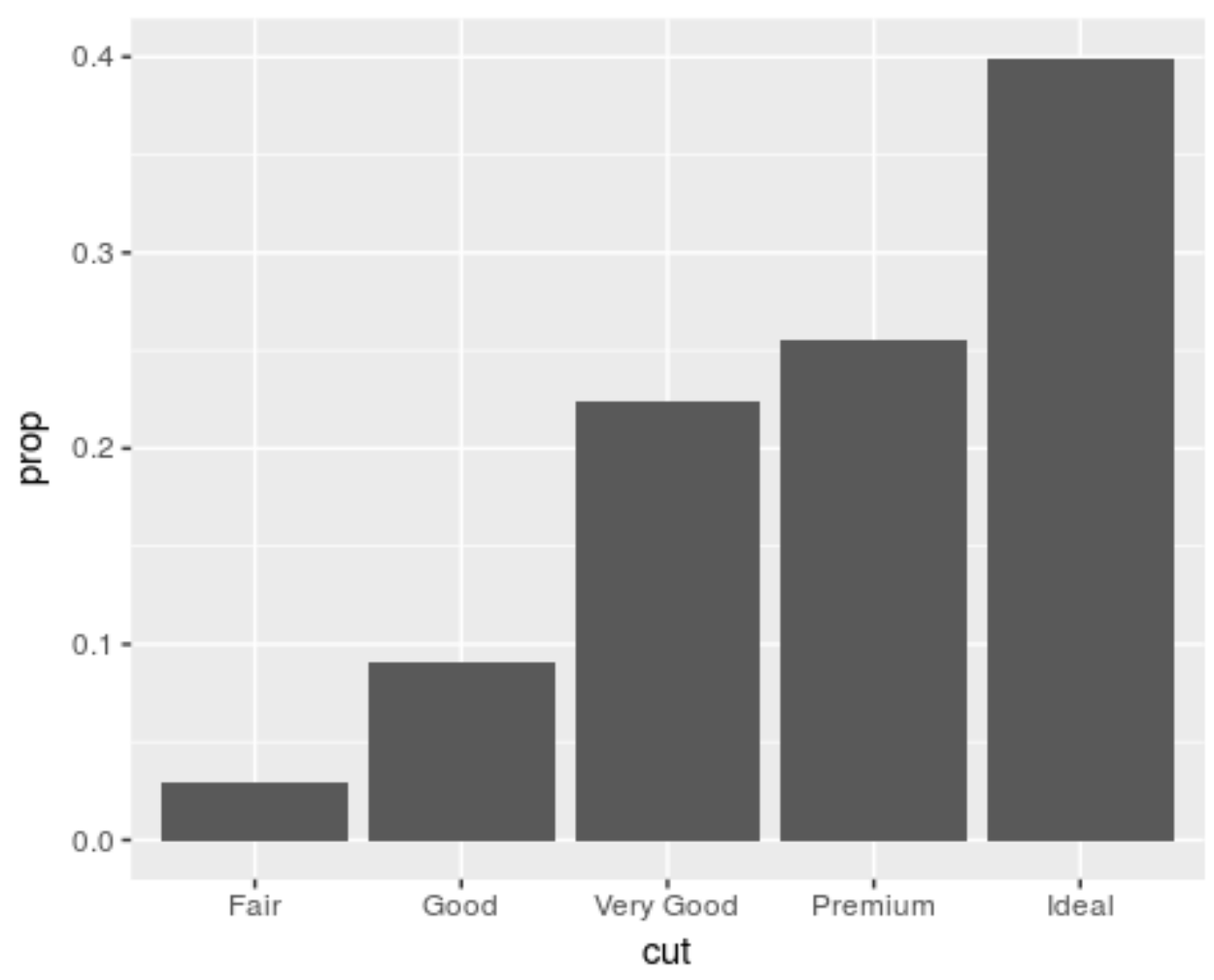

Figure 11.16 Bar chart with y-axis in proportion

To find the variables computed by the stat, look for the help section titled "computed variables."

3. You might want to draw greater attention to the statistical transformation in your code. For example, you might use stat_summary(), which summarises the $y$ values for each unique $\mathrm{x}$ value, to draw attention to the summary that you're computing:

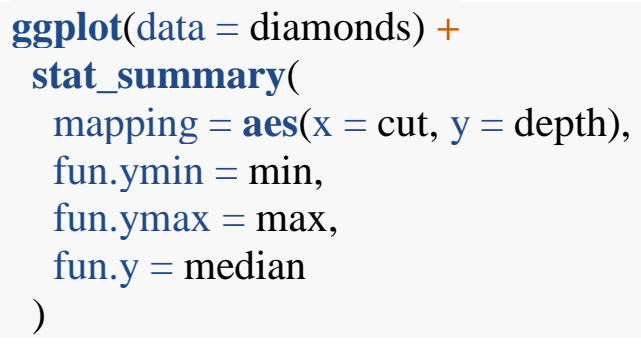




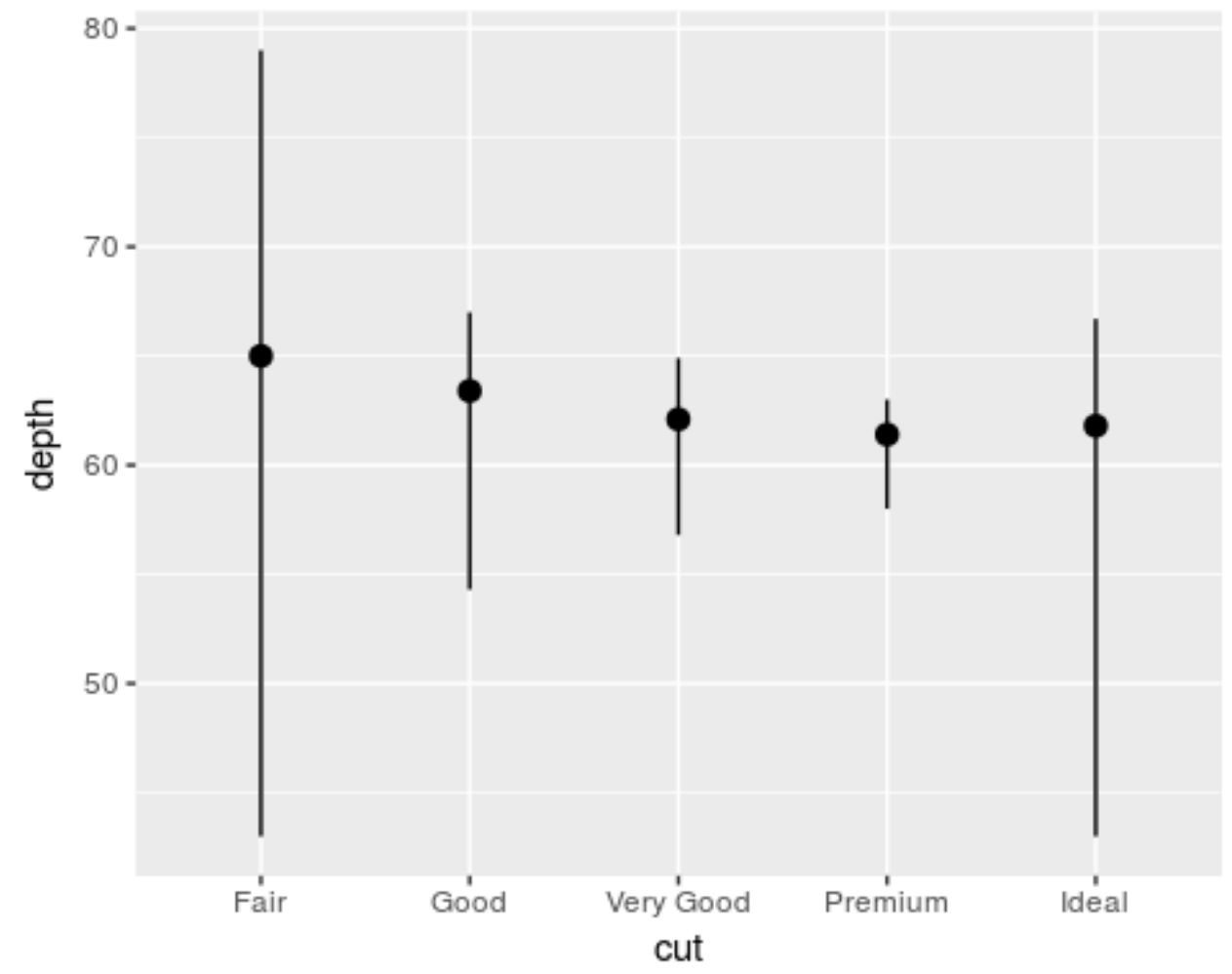

Figure 11.17 Chart with stat-summary

ggplot2 provides over 20 stats for you to use. Each stat is a function, so you can get help in a usual way (e.g., ?stat_bin). To see a complete list of stats, try the ggplot2 cheatsheet.

\subsubsection{Position Adjustments}

There's one more piece of magic associated with bar charts. You can color a bar chart using either the color aesthetic, or more usefully, fill:

ggplot $($ data $=$ diamonds $)+$

geom_bar(mapping $=$ aes $(\mathrm{x}=$ cut, colour $=$ cut $))$

ggplot(data $=$ diamonds $)+$

geom_bar(mapping $=\operatorname{aes}(\mathrm{x}=$ cut, fill $=$ cut $))$ 

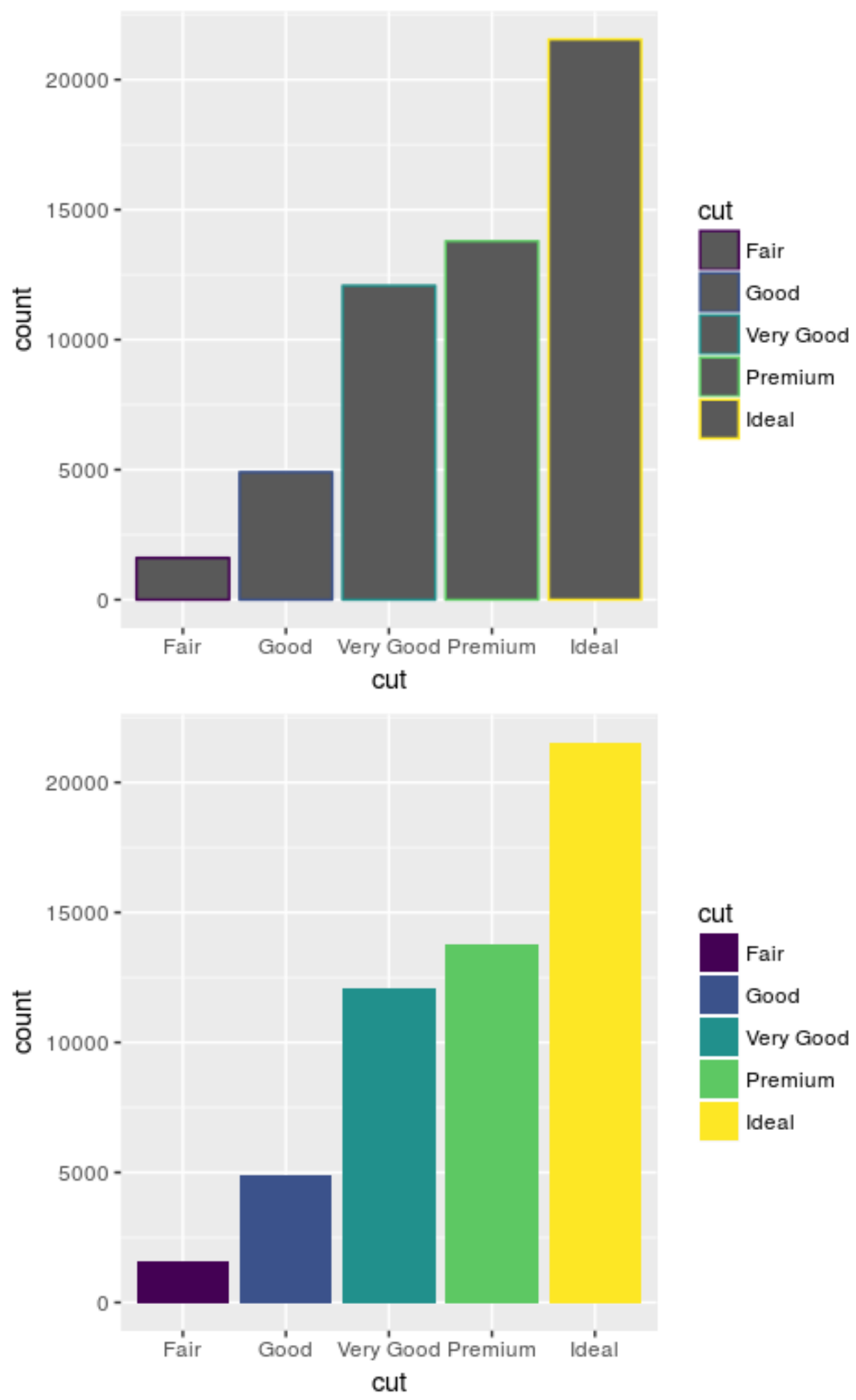

Figure 11.18 Bar chart with fill and color 
Note what happens if you map the fill aesthetic to another variable, like clarity: the bars are automatically stacked. Each colored rectangle represents a combination of cut and clarity.
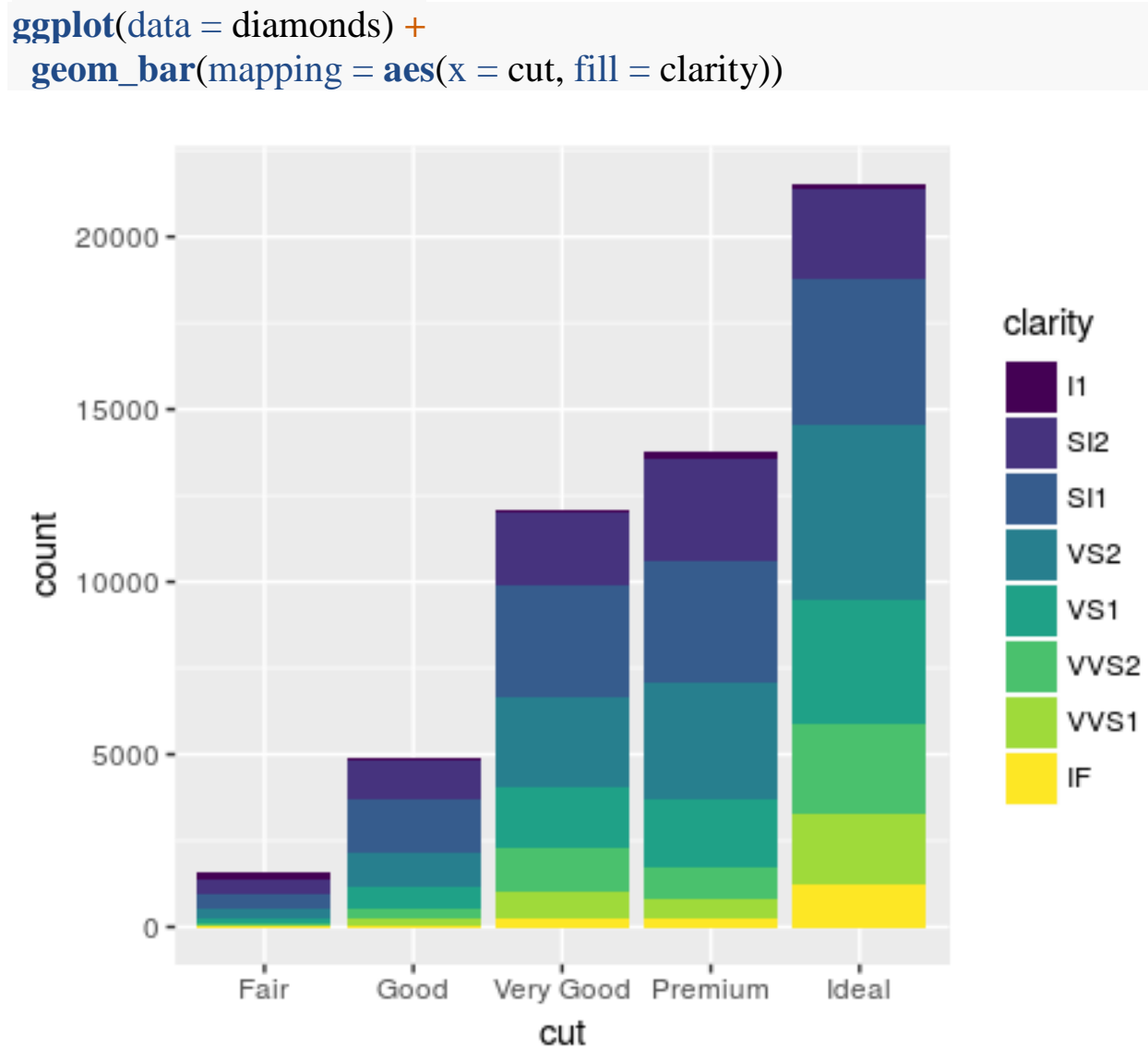

Figure 11.19 Stacked bar chart of diamonds by cut and clarity

The stacking is performed automatically by the position adjustment specified by the position argument. If you don't want a stacked bar chart, you can use one of three other options: "identity," "dodge" or "fill,"

- $\quad$ position = "identity" will place each object exactly where it falls in the context of the graph. This is not very useful for bars because it overlaps them. To see that overlapping we either need to make the bars slightly transparent by setting alpha to a small value or completely transparent by setting fill $=$ NA.

$\operatorname{ggplot}($ data $=$ diamonds, mapping $=\operatorname{aes}(\mathrm{x}=$ cut, fill $=$ clarity $))+$ geom_bar(alpha $=1 / 5$, position = "identity")

$\operatorname{ggplot}($ data $=$ diamonds, mapping $=$ aes $(\mathrm{x}=$ cut, colour $=$ clarity $))+$ geom_bar(fill = NA, position = "identity") 

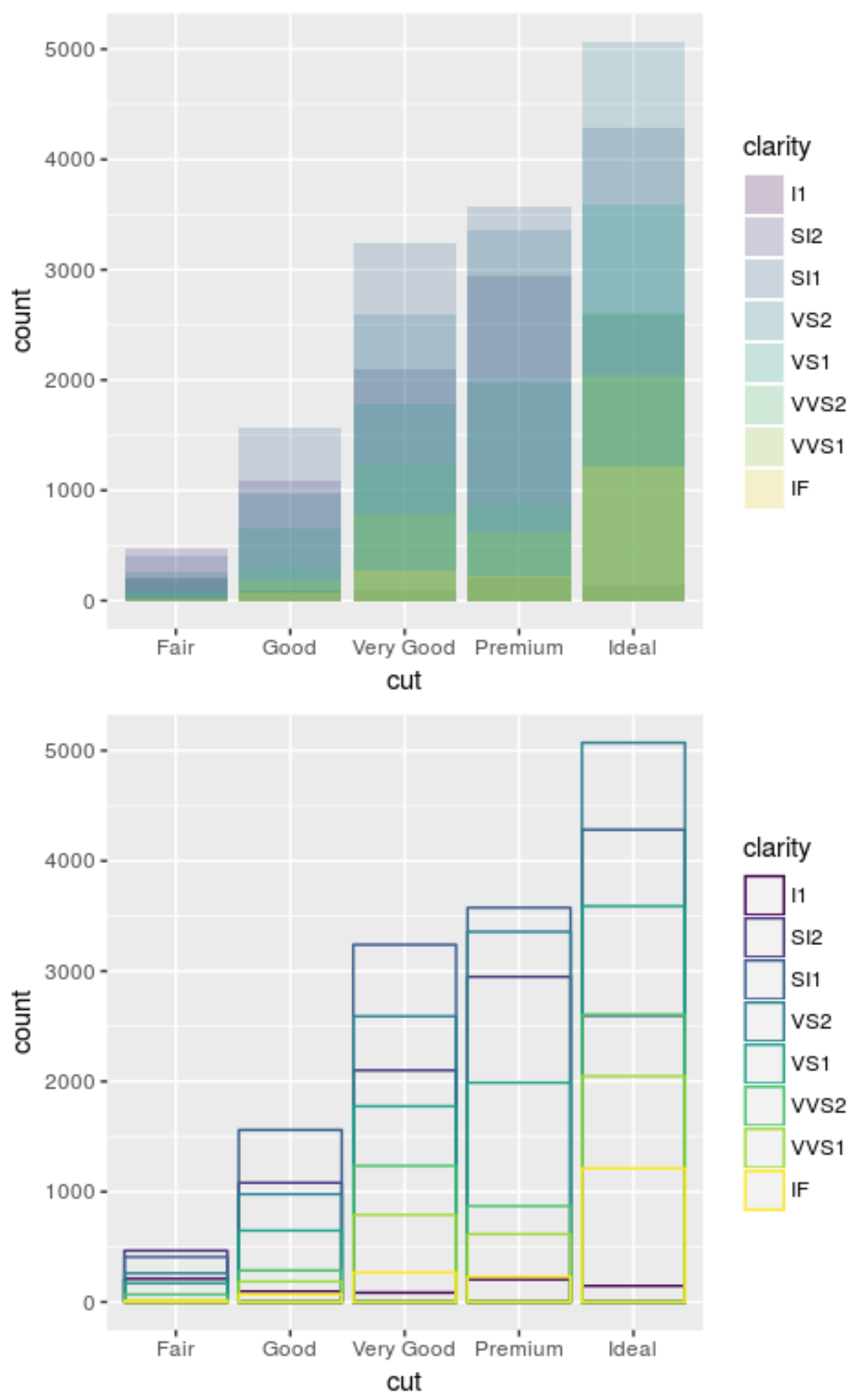

Figure 11.20 Bar chart with position='identity' 
The identity position adjustment is more useful for $2 \mathrm{~d}$ geoms, like points, where it is the default.

- $\quad$ position = "fill" works like stacking, but makes each set of stacked bars the same height. This makes it easier to compare proportions across groups.

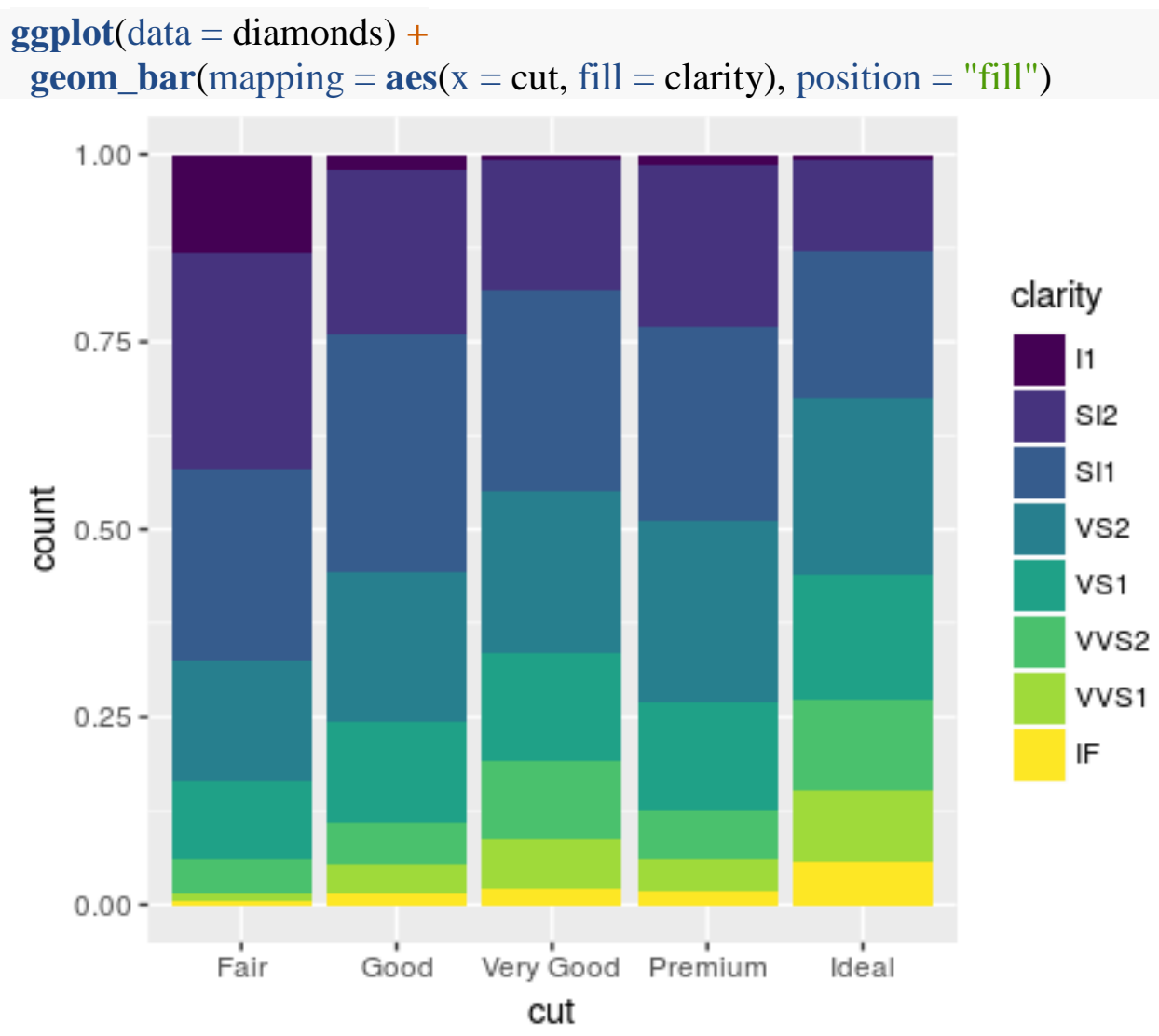

Figure 11.21 Bar chart with position='fill'

- $\quad$ position = "dodge" places overlapping objects directly beside one another. This makes it easier to compare individual values.

ggplot $($ data $=$ diamonds $)+$

geom_bar(mapping = aes(x = cut, fill = clarity), position = "dodge") 


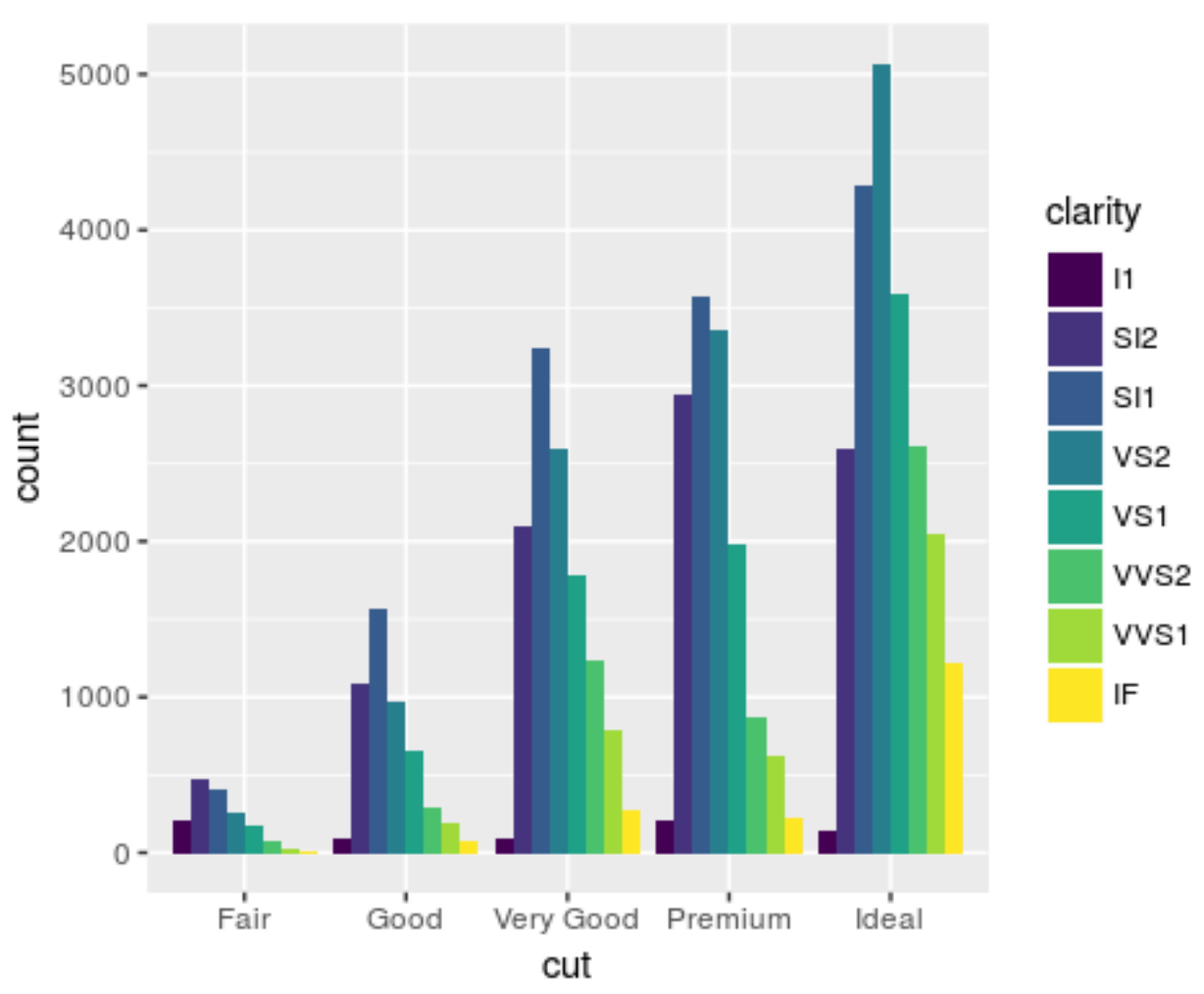

Figure 11.22 Bar chart with position='identity'

There's one other type of adjustment that's not useful for bar charts, but it can be very useful for scatter plots. Recall our first scatter plot. Did you notice that the plot displays only 126 points, even though there are 234 observations in the dataset? 


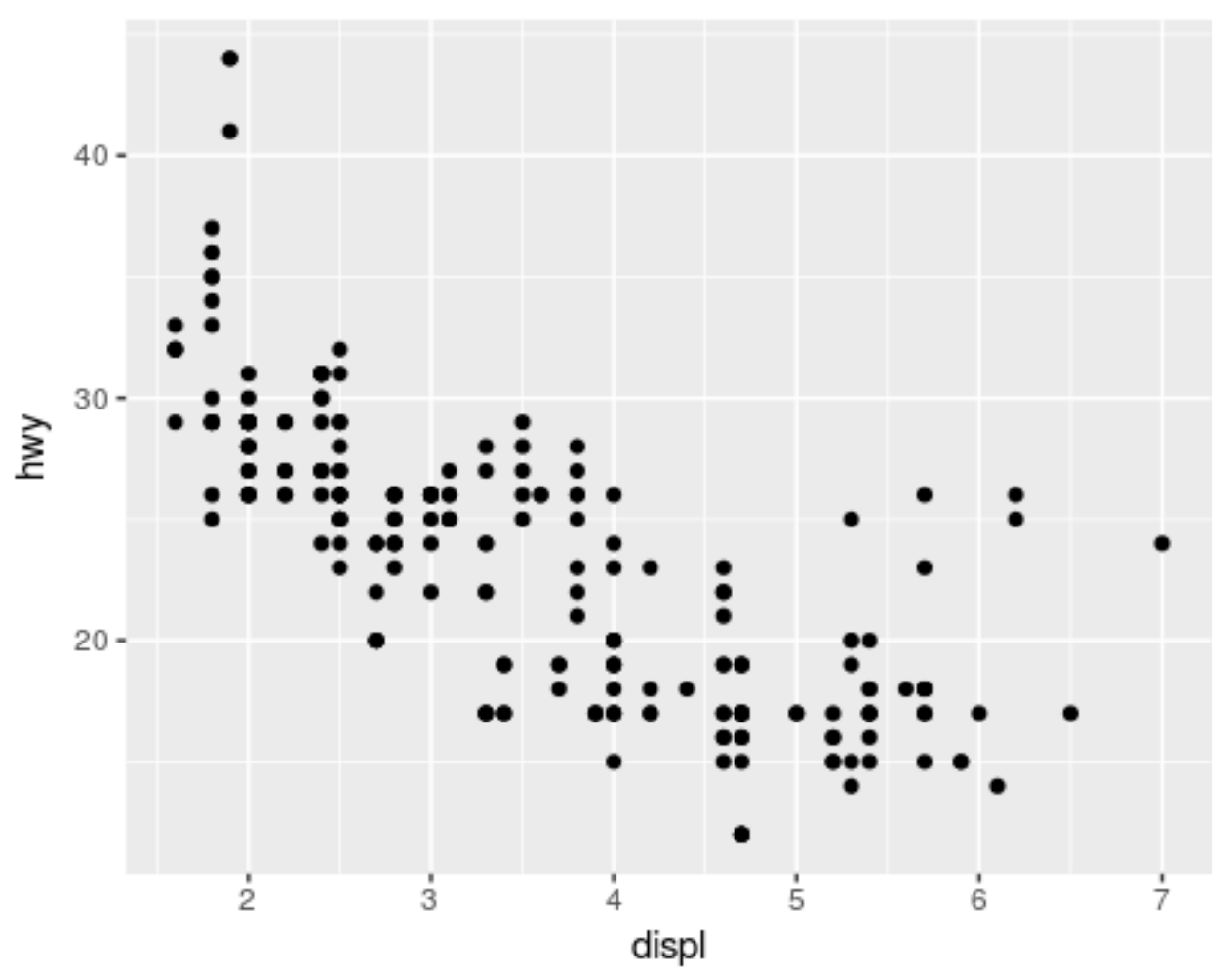

Figure 11.23 Scatter plot of highway mpg by displ

The values of hwy and displ are rounded so the points appear on a grid and many points overlap each other. This problem is known as overplotting. This arrangement makes it hard to see where the mass of the data is. Are the data points spread equally throughout the graph, or is there one special combination of hwy and displ that contains 109 values?

You can avoid this gridding by setting the position adjustment to "jitter." Position = "jitter" adds a small amount of random noise to each point. This spreads the points out because no two points are likely to receive the same amount of random noise.

$\operatorname{ggplot}($ data $=\operatorname{mpg})+$

geom_point(mapping = aes $(\mathrm{x}=$ displ, $\mathrm{y}=$ hwy), position = "jitter") 


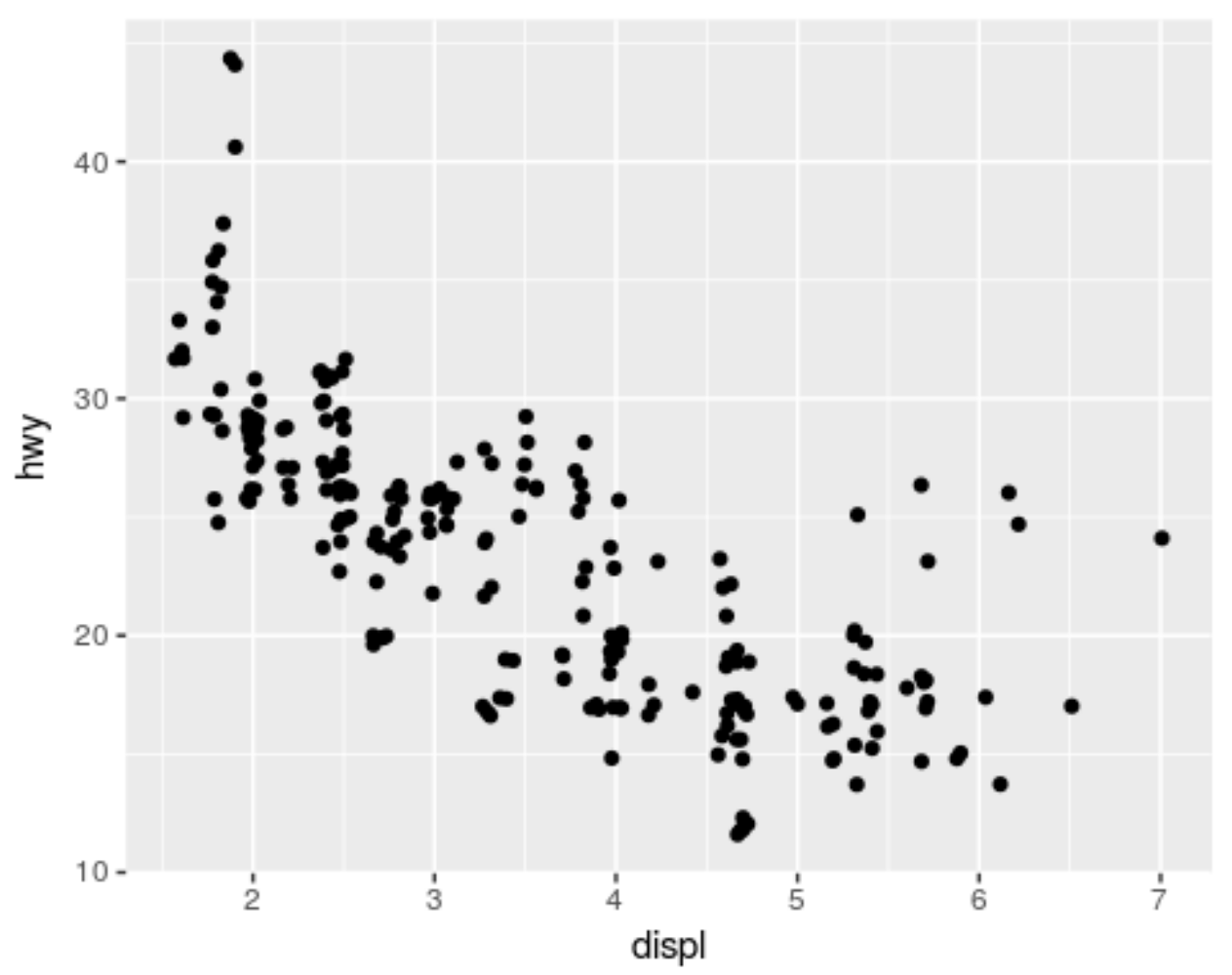

Figure 11.24 Scatter plot of highway mpg by displ, with position='jitter'

Adding randomness seems like a strange way to improve your plot, but while it makes your graph less accurate at small scales, it makes your graph more revealing at large scales. Because this is such a useful operation, ggplot2 comes with a shorthand for geom_point(position = "jitter"): geom_jitter().

To learn more about a position adjustment, look up the help page associated with each adjustment: ?position_dodge, ?position_fill, ?position_identity, ?position_jitter, and ?position_stack.

\subsubsection{Coordinate Systems}

Coordinate systems are probably the most complicated part of ggplot2. The default coordinate system is the Cartesian coordinate system where the $\mathrm{x}$ and $\mathrm{y}$ position act independently to find the location of each point. There are a number of other coordinate systems that are occasionally helpful.

- coord_flip() switches the $\mathrm{x}$ - and y-axis. This is useful if, for example, you want horizontal boxplots. It's also useful for long labels: It's hard to get them to fit without overlapping on the $\mathrm{x}$-axis.

$\operatorname{ggplot}($ data $=$ mpg, mapping $=\operatorname{aes}(\mathrm{x}=$ class, $\mathrm{y}=\mathrm{hwy}))+$ geom_boxplot() 

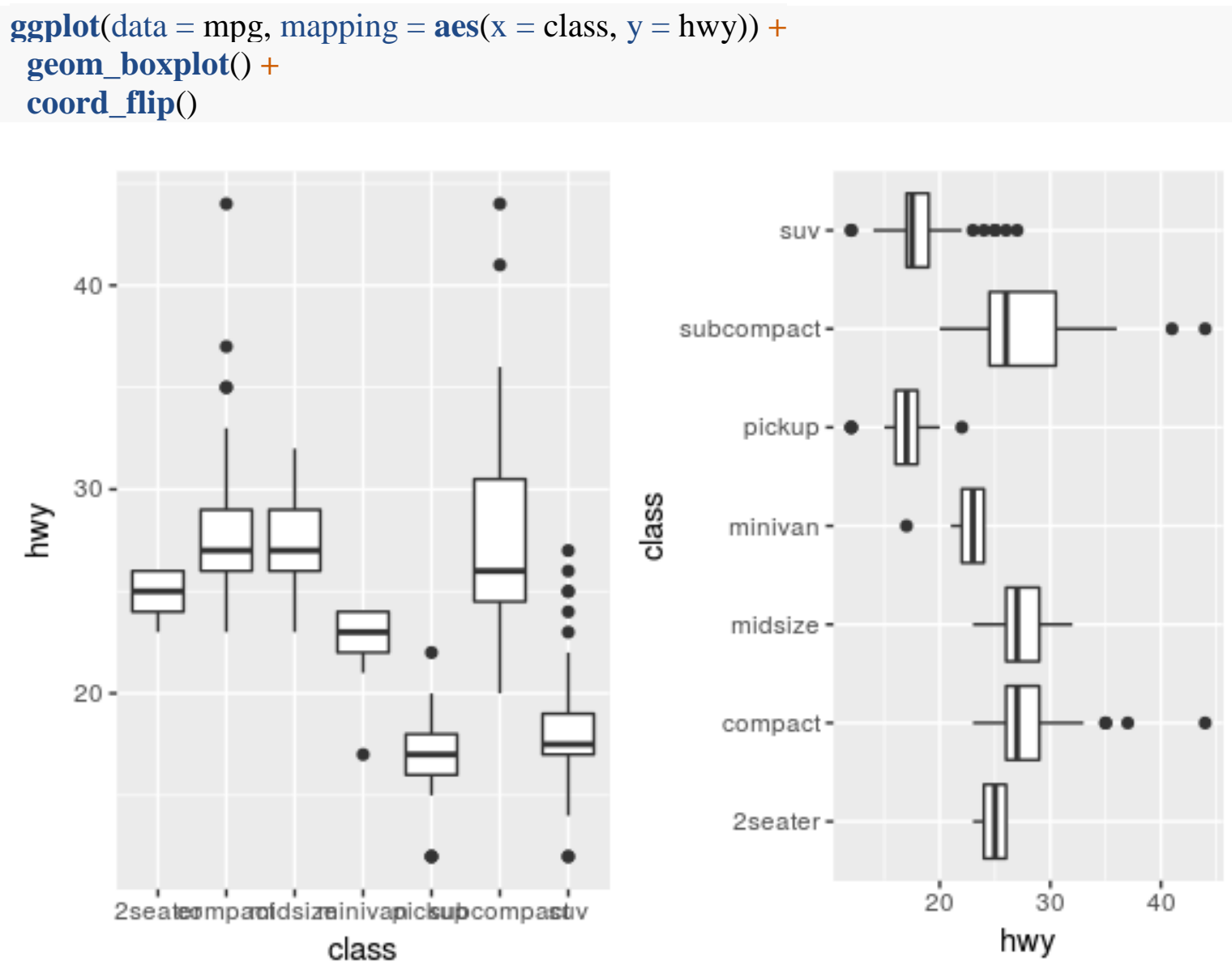

Figure 11.24 Bar chart with flipped - and y-axis

- $\quad$ coord_quickmap() sets the aspect ratio correctly for maps. This is very important if you're plotting spatial data with ggplot2.

nz <- map_data("nz")

ggplot(nz, aes(long, lat, group = group $))+$

geom_polygon(fill = "white", colour = "black")

ggplot(nz, aes(long, lat, group = group $))+$

geom_polygon(fill = "white", colour = "black") +

coord_quickmap () 

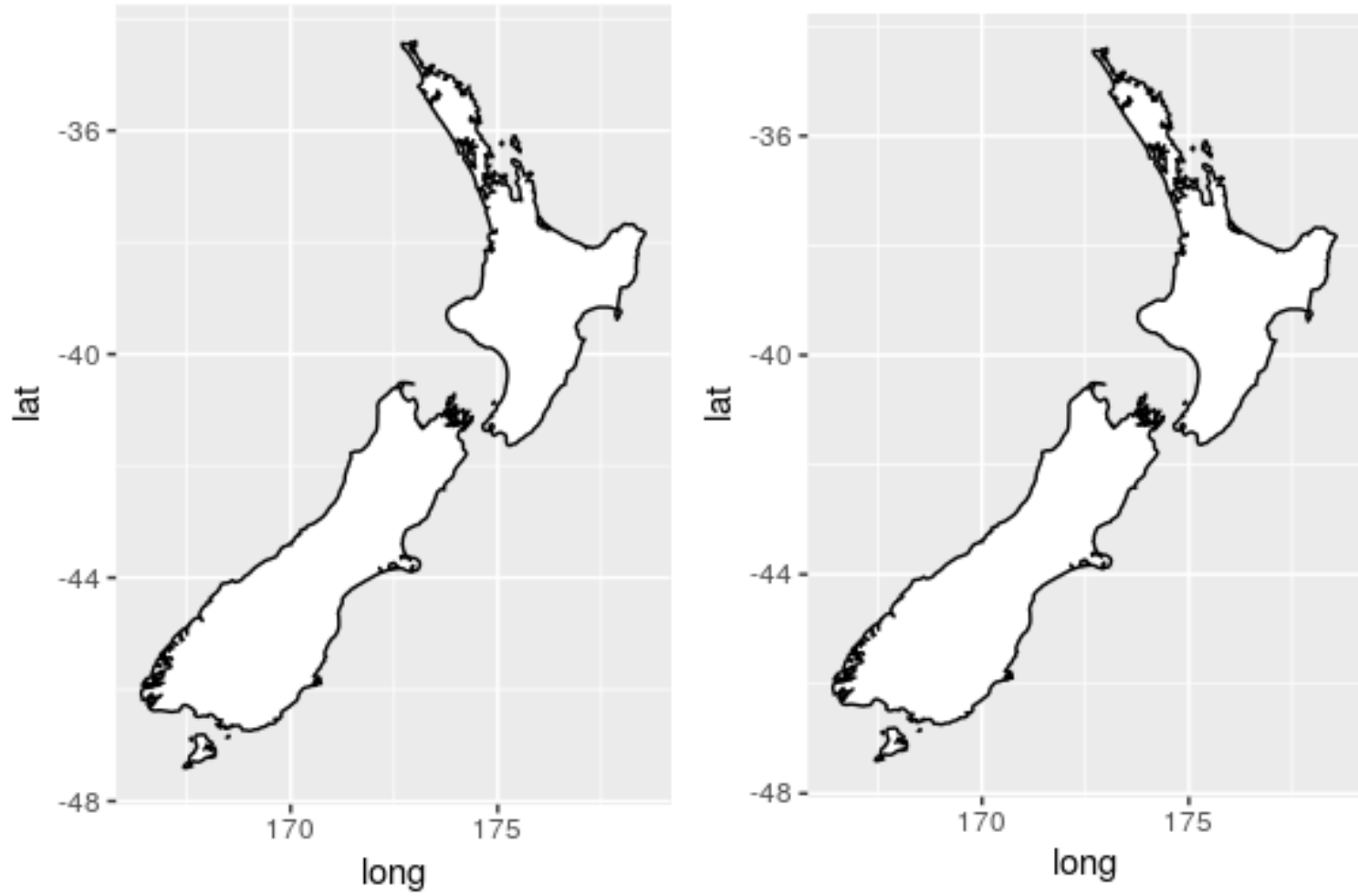

Figure 11.25 Map of New Zealand with ggplot2

- $\quad$ coord_polar() uses polar coordinates. Polar coordinates reveal an interesting connection between a bar chart and a Coxcomb chart.

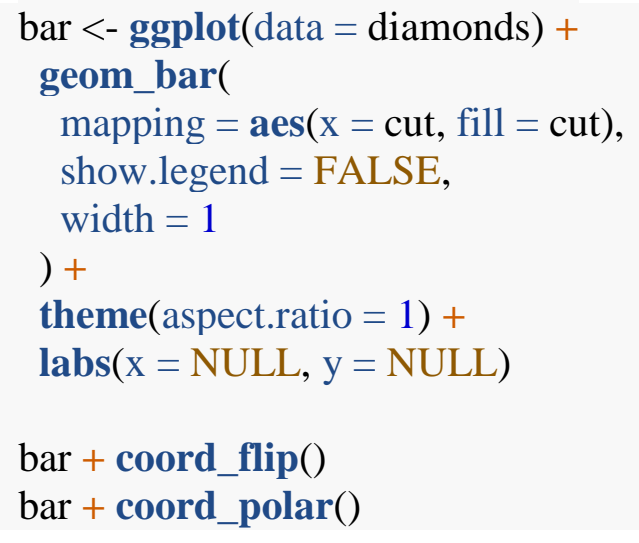



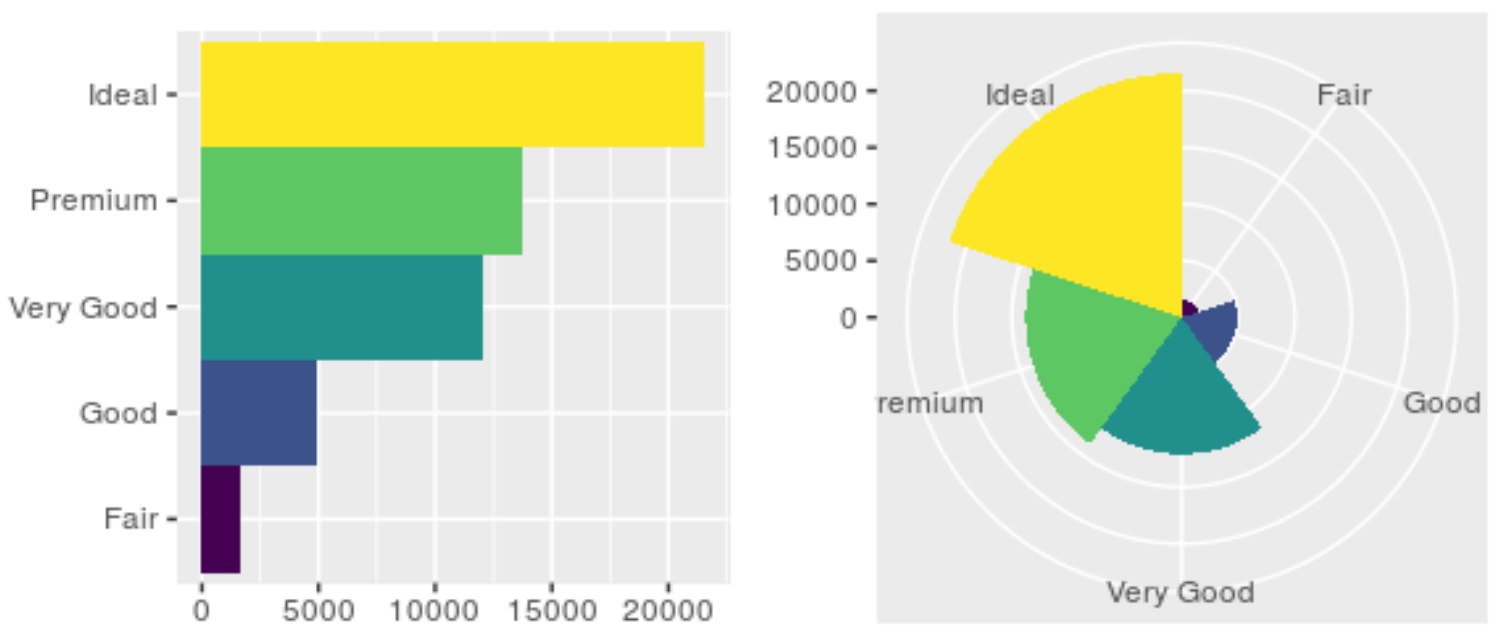

Figure 11.26 Coxcomb chart with the flipped and polar coordinate system

\subsubsection{The Layered Grammar of Graphics}

In the previous sections, you learned much more than how to make scatter plots, bar charts, and boxplots. You learned a foundation that you can use to make any type of plot with ggplot2. To see this, let's add position adjustments, stats, coordinate systems, and faceting to our code template:

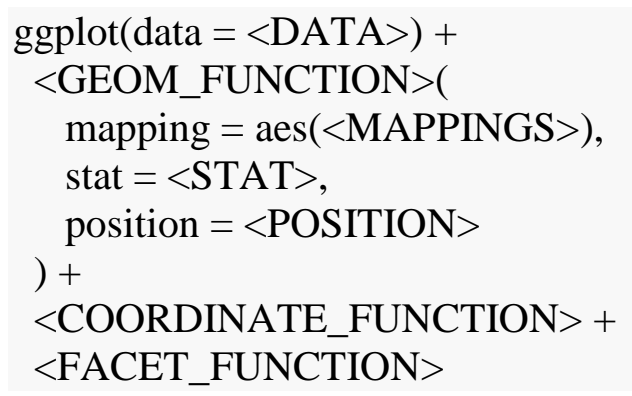

Our new template takes seven parameters, the bracketed words that appear in the template. In practice, you rarely need to supply all seven parameters to make a graph because ggplot2 will provide useful defaults for everything except the data, the mappings, and the geom function. The seven parameters in the template compose the grammar of graphics, a formal system for building plots. The grammar of graphics is based on the insight that you can uniquely describe any plot as a combination of a dataset, a geom, a set of mappings, a stat, a position adjustment, a coordinate system, and a faceting scheme.

To see how this works, consider how you could build a basic plot from scratch: You could start with a dataset and then transform it into the information that you want to display (with a stat). 


\section{Begin with the diamonds data set}

\section{Compute counts for each cut value with stat_count ( ).}

\begin{tabular}{|c|c|c|c|c|c|c|c|c|c|}
\hline carat & cut & color & clarity & depth & table & price & $\mathbf{x}$ & $\mathbf{y}$ & $\mathbf{z}$ \\
\hline 0.23 & Ideal & E & SI2 & 61.5 & 55 & 326 & 3.95 & 3.98 & 2.43 \\
\hline 0.21 & Premium & E & SI1 & 59.8 & 61 & 326 & 3.89 & 3.84 & 2.31 \\
\hline 0.23 & Good & E & VS1 & 56.9 & 65 & 327 & 4.05 & 4.07 & 2.31 \\
\hline 0.29 & Premium & I & VS2 & 62.4 & 58 & 334 & 4.20 & 4.23 & 2.63 \\
\hline 0.31 & Good & J & SI2 & 63.3 & 58 & 335 & 4.34 & 4.35 & 2.75 \\
\hline$\ldots$ & $\ldots$ & $\ldots$ & $\ldots$ & $\ldots$ & $\ldots$ & $\ldots$ & $\ldots$ & $\ldots$ & $\ldots$ \\
\hline
\end{tabular}

Figure 11.27 Visualization Grammar Step 1

Next, you could choose a geometric object to represent each observation in the transformed data. You could then use the aesthetic properties of the geoms to represent variables in the data. You would map the values of each variable to the levels of an aesthetic.

\begin{tabular}{|c|c|c|c|c|c|c|c|c|c|}
\hline carat & cut & color & clarity & depth & table & price & $\mathbf{x}$ & $\mathbf{y}$ & $\mathbf{z}$ \\
\hline 0.23 & Ideal & E & SI2 & 61.5 & 55 & 326 & 3.95 & 3.98 & 2.43 \\
\hline 0.21 & Premium & E & SI1 & 59.8 & 61 & 326 & 3.89 & 3.84 & 2.31 \\
\hline 0.23 & Good & E & VS1 & 56.9 & 65 & 327 & 4.05 & 4.07 & 2.31 \\
\hline 0.29 & Premium & I & VS2 & 62.4 & 58 & 334 & 4.20 & 4.23 & 2.63 \\
\hline 0.31 & Good & J & SI2 & 63.3 & 58 & 335 & 4.34 & 4.35 & 2.75 \\
\hline$\ldots$ & $\ldots$ & $\ldots$ & $\ldots$ & $\ldots$ & $\ldots$ & $\ldots$ & $\ldots$ & $\ldots$ & $\ldots$ \\
\hline
\end{tabular}
3. Represent each observation with a bar.

\section{Map the fill of each bar to} the .. count.. variable.

\section{stat_count0}

\begin{tabular}{|c|c|c|c|}
\hline cut & count & prop \\
\hline Fair & 1610 & 1 & $\longrightarrow$ \\
\hline Good & 4906 & 1 & $\longrightarrow$ \\
\hline Very Good & 12082 & 1 & $\longrightarrow$ \\
\hline Premium & 13791 & 1 & $\longrightarrow$ \\
\hline Ideal & 21551 & 1 & $\longrightarrow$ \\
\hline
\end{tabular}

Figure 11.28 Visualization Grammar Step 2 
You'd then select a coordinate system to place the geoms into. You'd use the location of the objects (which is itself an aesthetic property) to display the values of the $\mathrm{x}$ and $\mathrm{y}$ variables. At that point, you would have a complete graph, but you could further adjust the positions of the geoms within the coordinate system (a position adjustment) or split the graph into subplots (faceting). You could also extend the plot by adding one or more additional layers, where each additional layer uses a dataset, a geom, a set of mappings, a stat, and a position adjustment.

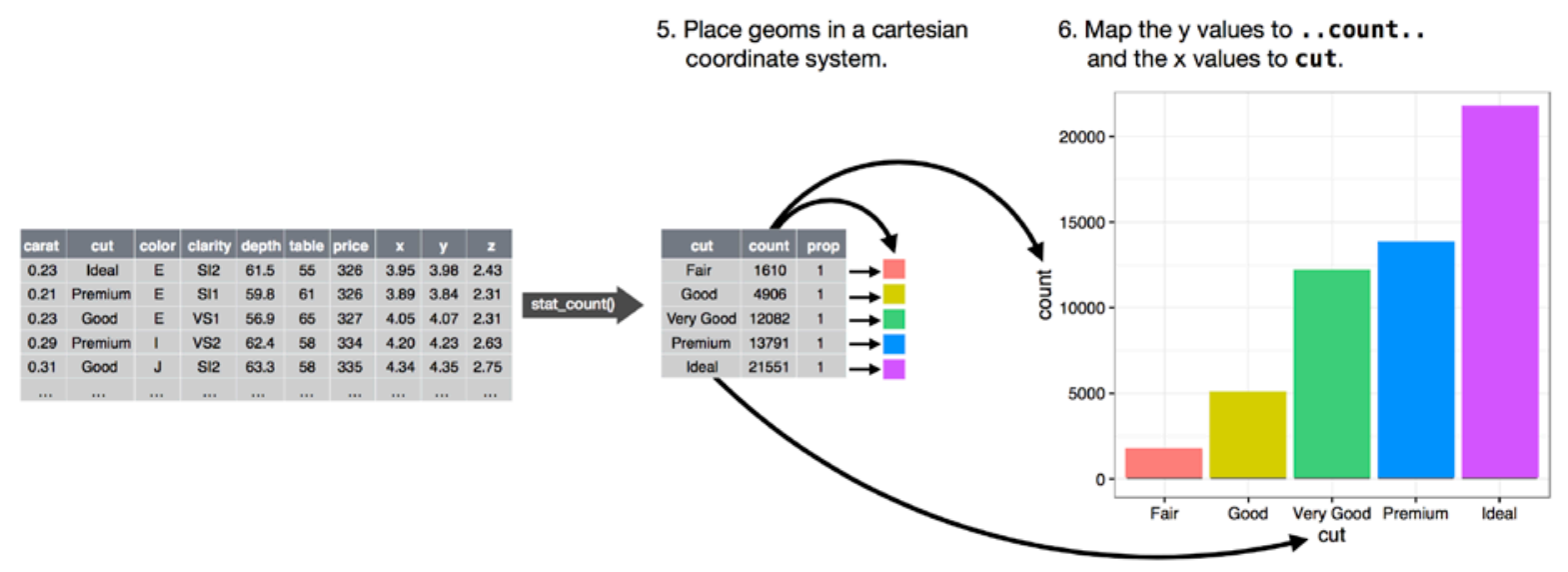

Figure 11.29 Visualization Grammar Step 3

You could use this method to build any plot that you imagine. In other words, you can use the code template that you've learned in this chapter to build hundreds of thousands of unique plots.

\subsubsection{Additional Resources}

1. ggfortify - plot some popular R packages with ggplot2

2. geom_map

3. geom_sf - ggplot2 working with simple feature and the simple feature package for geospatial analysis

4. ggmap - raster map tiles from popular online mapping services

5. interactive maps and charts with $\mathrm{R}$

\subsection{EXERCISE}

1. Plot the daily bike counts for Hawthorne and Tilikum with ggplot2; experiment with different options and select the one works best for you;

2. Plot weekly, monthly, and annual bike counts for Hawthorne and Tilikum;

3. Now think about and try out different ways to plot weather information along with your daily bike counts (separately or together); and

4. You can combine time series stats and ggplot2/ggfortify to plot seasonal variation, trend and noise separately. See here for an example of how to do this. 


\subsection{LEARNING MORE:}

1. ggplot2 tutorial by Prof. Jenny Bryan

2. R4DS Chapter 28. Graphics for communication

3. The ggplot2 Cheatsheet - RStudio

4. R Graphics Cookbook 


\subsection{SPLIT-APPLY-COMBINE PATTERN IN DATA MODELING}

\subsection{READINGS}

1. Wickham, Hadley, 2011. The Split-Apply-Combine Strategy for Data Analysis, Journal of Statistical Software, Volume 40, Issue 1.

2. R4DS Chapter 25. Many Models

If you are not familiar with the basic workflow of fitting models with $\mathrm{R}$, you should review two chapters:

1. R4DS Chapter 23. Model basics

2. $\quad$ R4DS Chapter 24. Model building

\subsection{SPLIT-APPLY-COMBINE}

A common analytical pattern is to:

- $\quad$ split data into pieces,

- $\quad$ apply some function to each piece, and

- combine the results back together again.

Generally, avoid using loops when you need to do Split-Apply-Combine, consider these alternatives instead:

1. Entry level: dplyr::group_by()

2. General approach: nesting

3. *apply functions and plyr package (non-tidyverse solution)

\subsection{LESSON}

The lesson below is adapted from UBC's STAT 545 by Professor Jenny Bryan.

\subsubsection{Think Before You Create Excerpts of Your Data ...}

If you feel the urge to store a little snippet of your data:

snippet <- my_big_dataset $\%>\%$

filter(some_variable == some_value)

\#\# or

snippet <- subset(my_big_dataset, some_variable == some_value)

Stop and ask yourself ...

Do I want to create mini datasets for each level of some factor (or a unique combination of several factors) ... in order to compute or graph something? If YES, use proper data aggregation techniques or facetting in ggplot2 -- don't subset the data. Or, more realistically, 
subset the data only as a temporary measure while you develop your elegant code for computing on or visualizing these data subsets.

If NO, then maybe you really do need to store a copy of a subset of the data. But seriously consider whether one of these strategies that subset at "compute time" will get the job done:

- $\quad$ Pass an intact data frame and use the subset = argument of a command. Many functions have it!

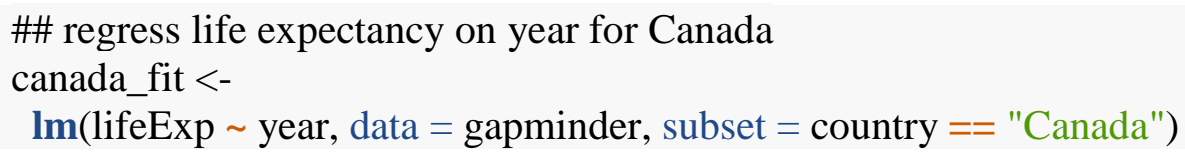

- $\quad$ Pipe filtered data into a command. A very general solution.

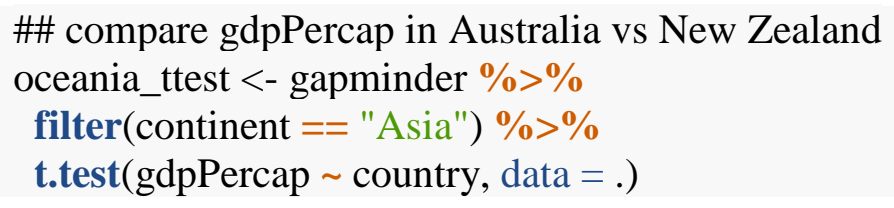

Creating copies and excerpts of your data clutters up your workspace, script and mind, often leading to confusion and mistakes. It can be useful during development, but try to eliminate this as you polish your analysis.

\subsubsection{Prerequisite}

Load tidyverse and gapminder:

library(gapminder)

library(tidyverse)

\subsubsection{Split-Apply-Combine}

A common analytical pattern is to

- $\quad$ split data into pieces,

- $\quad$ apply some function to each piece, and

- combine the results back together again.

$\mathrm{R}$ has specialized tools for this that are much nicer than whatever DIY approach you might be contemplating. Especially if it involves for() loops. There's nothing inherently wrong or evil about for() loops, but you will spend a lot of time and characters on bookkeeping tasks. That can be a great way to pilot a computation, because it is so blessedly concrete. But consider revisiting your implementation with higher-level data aggregation tools once you've established proof-ofprinciple.

In base $\mathrm{R}$, these are the "apply" functions, such as apply(), aggregate(), tapply(), and by(). We prefer to use similar tools from the tidyverse, due to a more consistent interface, analysis-friendly output, and a more concise way to describe what to compute. The Hadley Wickham article in the 
readings — the split-apply-combine strategy for data analysis — provides a good high-level overview, but know that the tidyverse approaches outlined here now supersede the plyr package described there.

\subsubsection{Entry Level: dplyr::group_by()}

The most lightweight solution is given by dplyr::group_by(). group_by() adds some extra grouping structure to a data frame, based on levels of one or more categorical variables, but leaves the data frame intact. Then you can do group-wise computations using various functions in the tidyverse that automatically honor these groups.

The main function here is summarized (), which collapses each group into a single row in a new group-summarized data frame.

When does this break down? If anything you want to compute for a group is more complicated than, say, a single number, you have outgrown dplyr::summarize(). Example: Getting the range of life expectancies seen for each continent doesn't work with the code below.

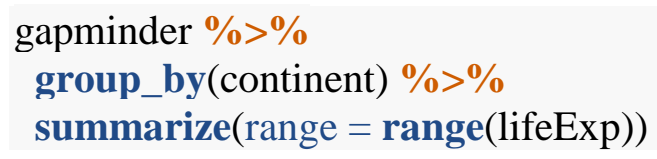

Now, in this case, we could reframe this in a summarize()-friendly form, but that doesn't work in general.

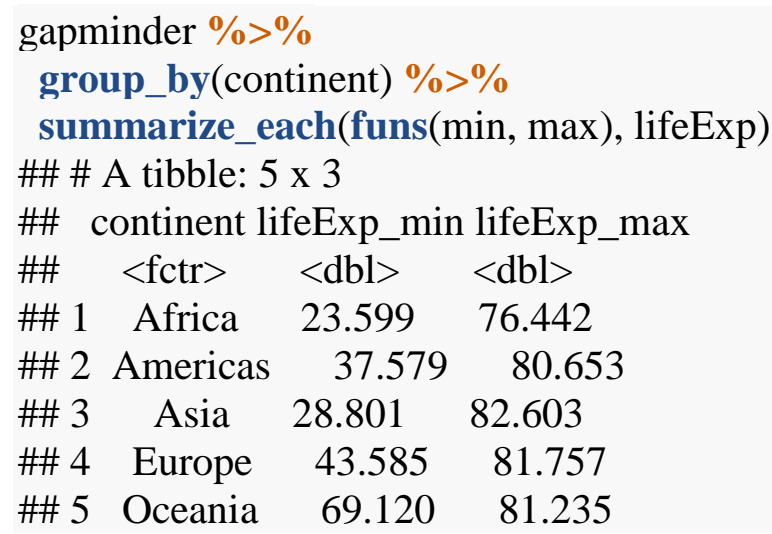

\subsubsection{General Approach: Nesting}

We use nesting as an extension of grouping in order to support more general group-wise computation. The collapse to a single row per group happens right away here, unlike the simple grouping above. When you nest data, the non-grouping variables are packaged into groupspecific data frames that are held in a special variable called a list-column. You then apply your computation to the components of this list (i.e., the data frames). List-columns take a little getting used to, but the payoff is huge. 
Let's get ready to fit a model for each country in the Gapminder dataset. First, we group, as above, and then nest. We group by country and continent in order to keep those two variables "on the outside."

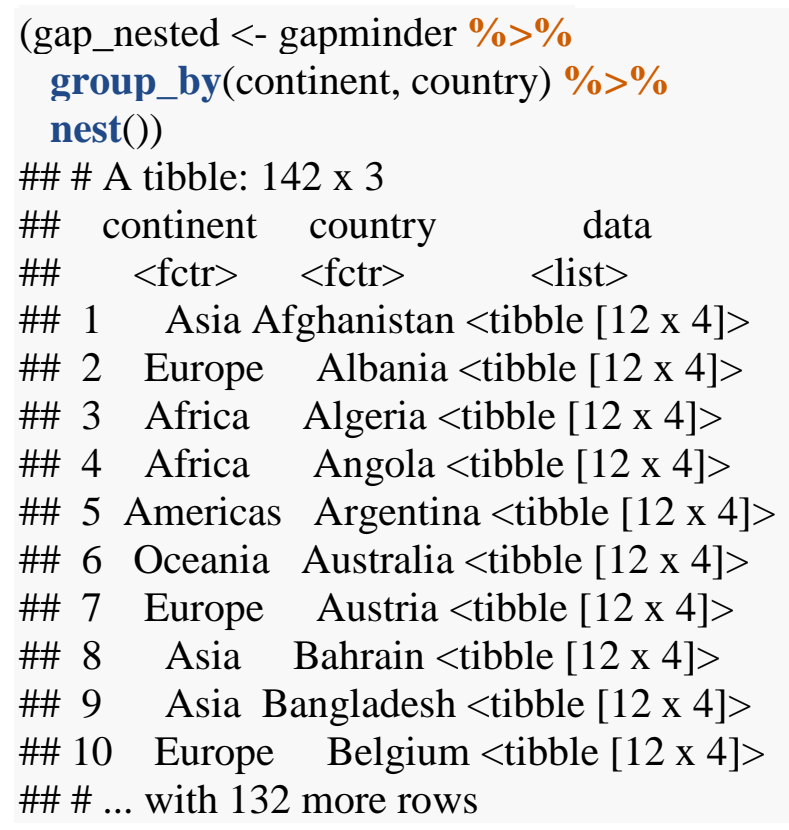

What do you notice?

- The immediate collapse to 142 rows, one per country.

- The familiar presence of continent and country.

- The unfamiliar look of the new data variable, which is ... a list! It is a list-column of continent-specific tibbles.

How on earth do you inspect it?!? In RStudio, gap_nested \%>\% View() is often helpful (moderately so in this case). It's often nicer to inspect a single element like so:

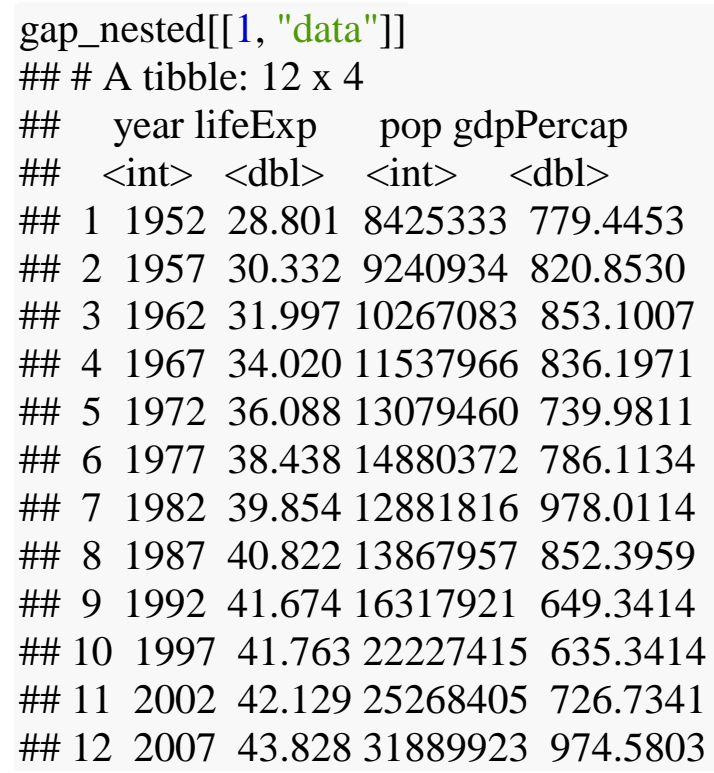


Here double square brackets are used to extract a single element and they are simplifying. An equivalent call is gap_nested[["data"]][[1]] (which I find even more opaque) or gap_nested\$data[[1]] (which is pretty nice). We're looking at the first of 142 country-specific data frames, which happen to be for Afghanistan.

The presence of list-columns is always a temporary, uncomfortable state.

\subsubsection{Apply a Function purrr::map() and mutate()}

How do we now iterate over the elements of gap_nested\$data? It is a list, so we use general approaches for applying a function to each element of a list. In base R, this means lapply(), but we will use the tidyverse function purrr::map(), which has a few advantages described elsewhere. Walk before you run (i.e., do an example first)!

Let's fit a model to the data from Afghanistan. The form of the right-hand side is so that our intercept has a nice interpretation.

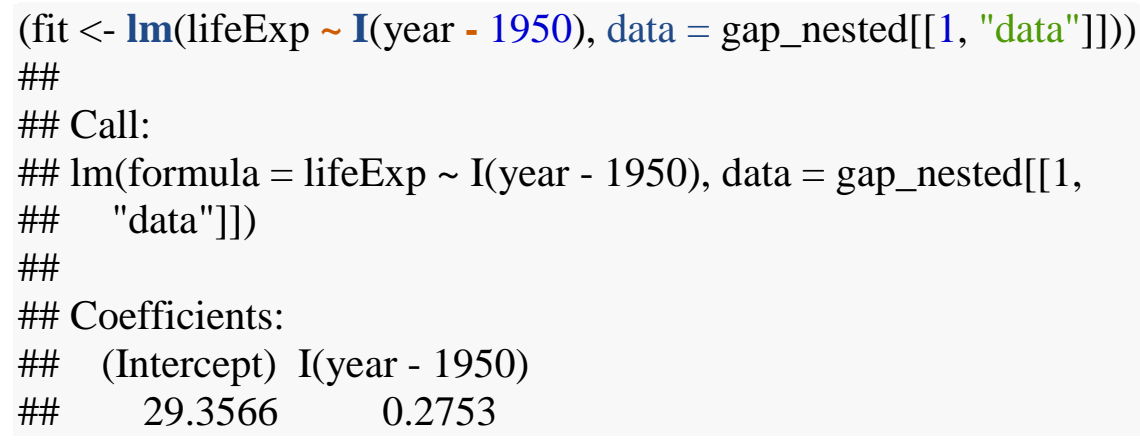

Let's capture that logic in a function and informally test that it returns the same results for Afghanistan.

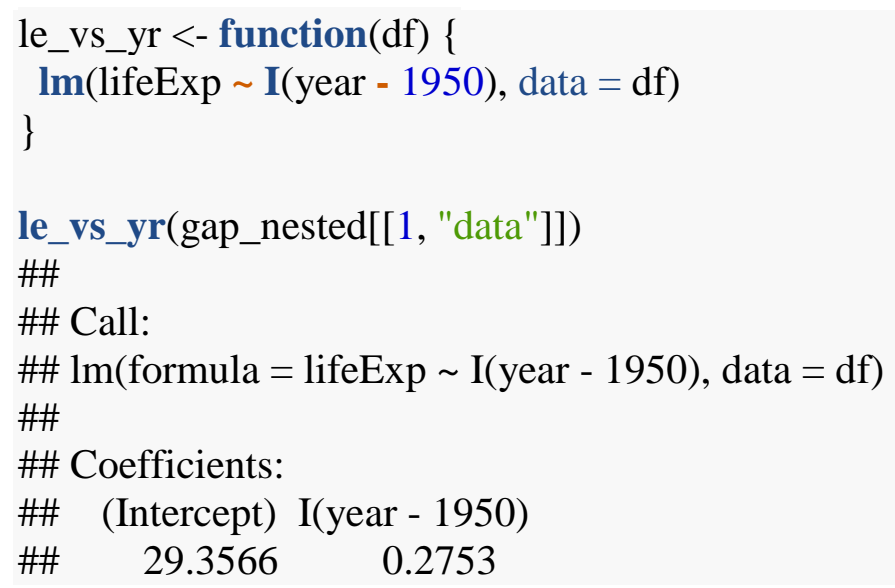

After you walk, jog before you run. Use purrr::map() to apply the fitting function le_vs_yr() to the first two elements of gap_nested\$data. 


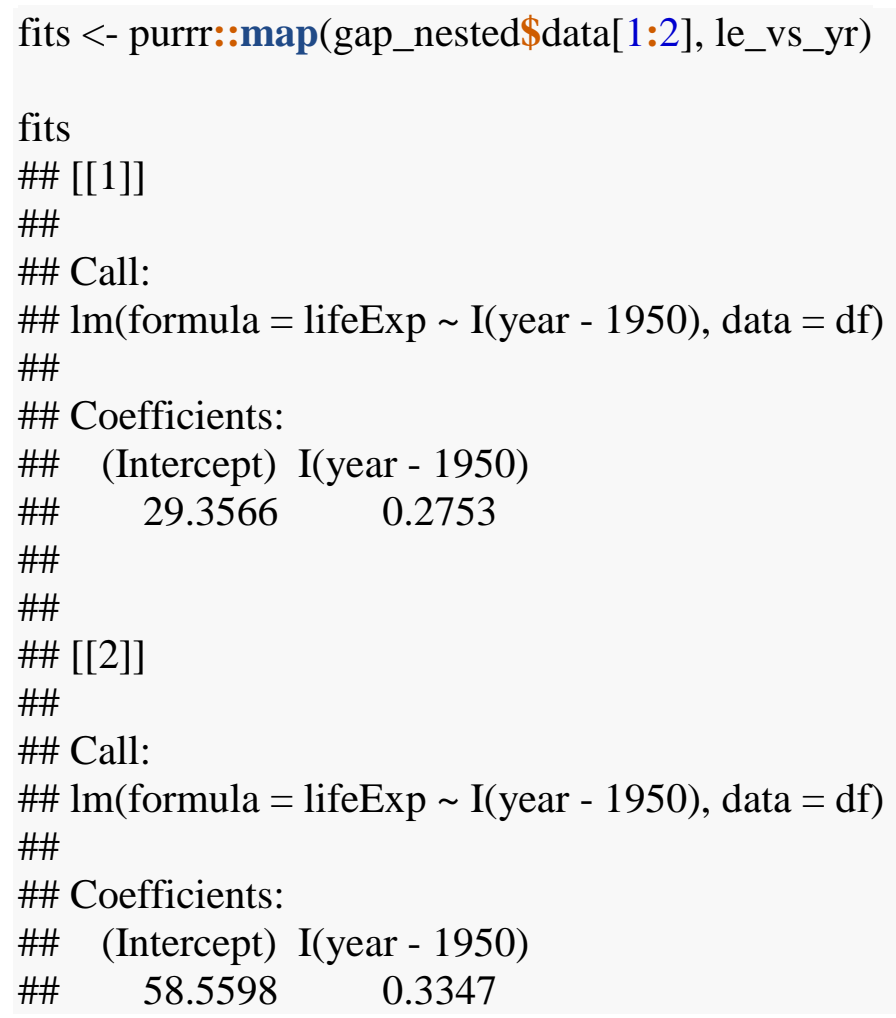

So, how do we run (i.e., scale this up to all countries)? And where do we put these fitted models? We'll use purrr::map() inside mutate(), meaning we store the models inside gap_nested in another list-column.

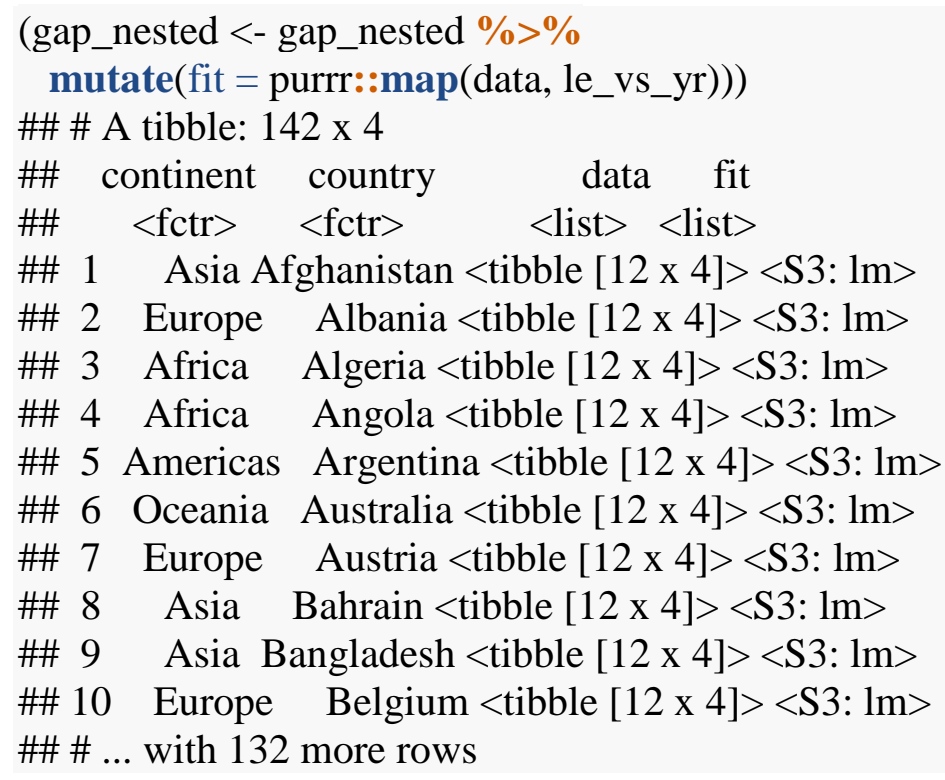

We have a new list-column, fit, that is even more intimidating than the previous, data. The fit variable holds 142 fitted linear models. What shall we do with that? 


\subsubsection{Always Have an Exit Strategy}

The list-column state is an uncomfortable and temporary one. The goal is always to pull information out of these complicated objects and package it as something simpler, usually a tibble. You always want a list-column exit strategy!

We are not afraid to fit linear models in this example, because of a fantastic package from David Robinson, broom, that provides exactly this for $\operatorname{lm}()$ and many other functions.

broom provides three key functions that take a $\operatorname{lm}()$ fit as input and give a useful tibble back:

- $\quad$ tidy(): a tidy version of summary() (e.g., a table with one row per parameter estimate)

- $\quad$ augment(): the original data, augmented with columns such as fitted values and residuals

- $\quad$ glance(): a one-row model summary

All of these are much friendlier than a fitted $\operatorname{lm}()$ object and set us up for interesting downstream analyses and plots.

\subsubsection{Simplify and Combine}

Let's look at the result of broom::tidy() for a single model. Walk before you run.

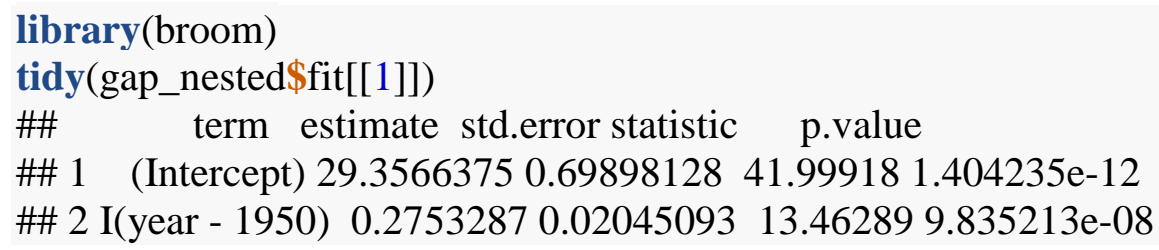

We get a two-row data frame, one with results for the intercept and one for the slope. This is much more approachable than fitted lm objects!

Apply tidy() to the model for each country with the same purrr::map() inside mutate() strategy as above.

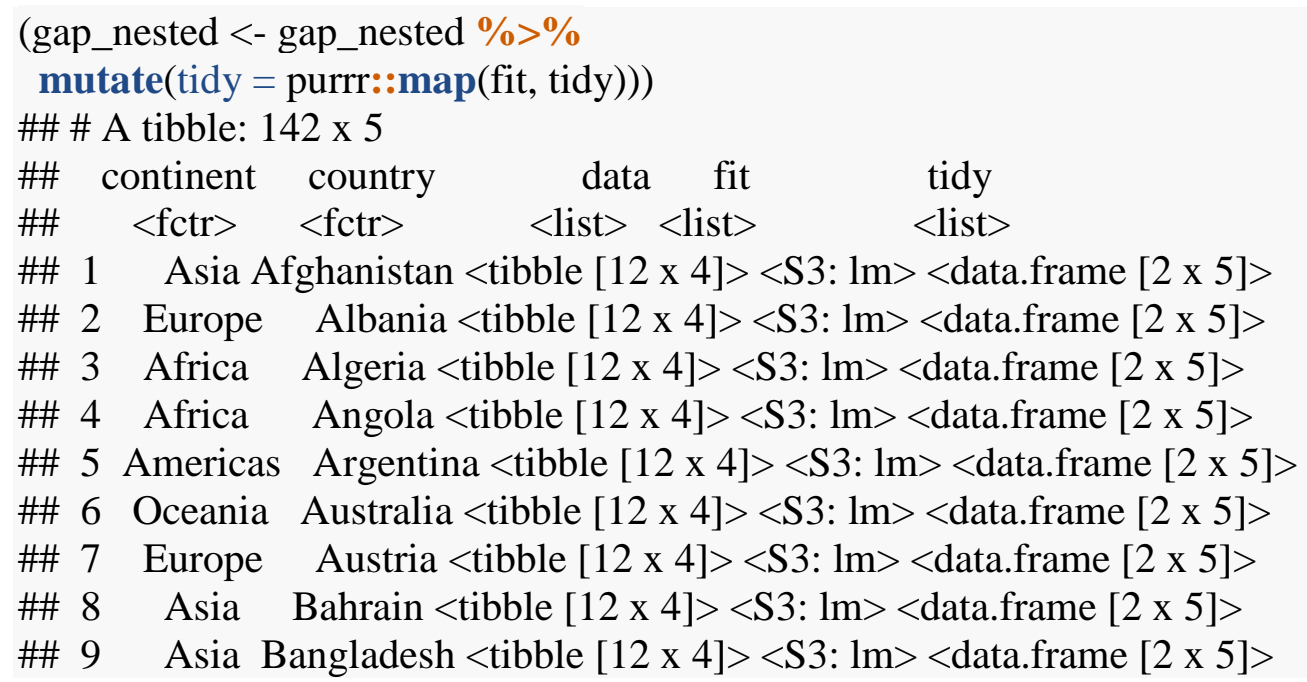


\#\# 10 Europe Belgium <tibble [12 x 4]><S3: $\operatorname{lm}><$ data.frame [2 x 5] $>$ \#\# \# ... with 132 more rows

The last step is now to simplify, preferably back to a normal tibble. We do this by retaining variables that are amenable to simplification and using unnest(), thus completing the circle.

(gap_coefs <- gap_nested $\%>\%$

select(continent, country, tidy) $\%>\%$

unnest(tidy))

\#\# \# A tibble: 284 x 7

\#\# continent country term estimate std.error statistic

\#\# $\quad<$ fctr $>\quad<$ fctr $>\quad<$ chr $>\quad<$ dbl $>\quad<$ dbl $>\quad<$ dbl $>$

\#\# 1 Asia Afghanistan (Intercept) 29.35663750 .69898127841 .999176

\#\# 2 Asia Afghanistan I(year - 1950) 0.27532870 .02045093413 .462890

\#\# 3 Europe Albania (Intercept) 58.55976181 .13357581251 .659325

\#\# 4 Europe Albania I(year - 1950) 0.33468320 .03316638710 .091036

\#\# 5 Africa Algeria (Intercept) 42.23641490 .75626904055 .848399

\#\# 6 Africa Algeria I(year - 1950) 0.56927970 .02212707025 .727749

\#\# 7 Africa Angola (Intercept) 31.70797410 .80428746339 .423683

\#\# 8 Africa Angola I(year - 1950) 0.20933990 .0235320038 .895964

\#\# 9 Americas Argentina (Intercept) 62.22501910.167091314 372.401279

\#\# 10 Americas Argentina I(year - 1950) 0.23170840 .00488879147 .395847

\#\# \# ... with 274 more rows, and 1 more variables: p.value <dbl>

\subsubsection{Recap}

The whole point of this was to apply a computation to all the pieces of a dataset and glue the results back together. First, let's review all of our work so far in one place. It's remarkably compact.

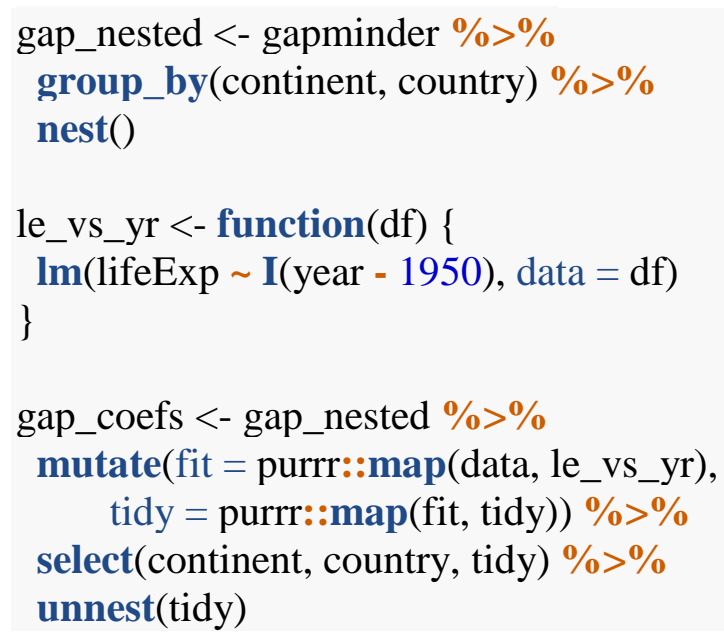

Reflect on how you would have obtained a data frame of country-specific intercepts and slopes from a regression of life expectancy on year. Did you know any approaches to solving this 
problem? If no, then rejoice that you now have one! If yes, does the approach outlined here seem simpler?

List-columns take some getting used to, but they are a required component of the strategy laid out above for general-purpose split-apply-combine.

\subsubsection{Enjoy the Payoff of Your Work}

Let's celebrate by exploring the estimated slopes and intercepts a bit. First, we recode the variables corresponding to "intercept" and "slope."

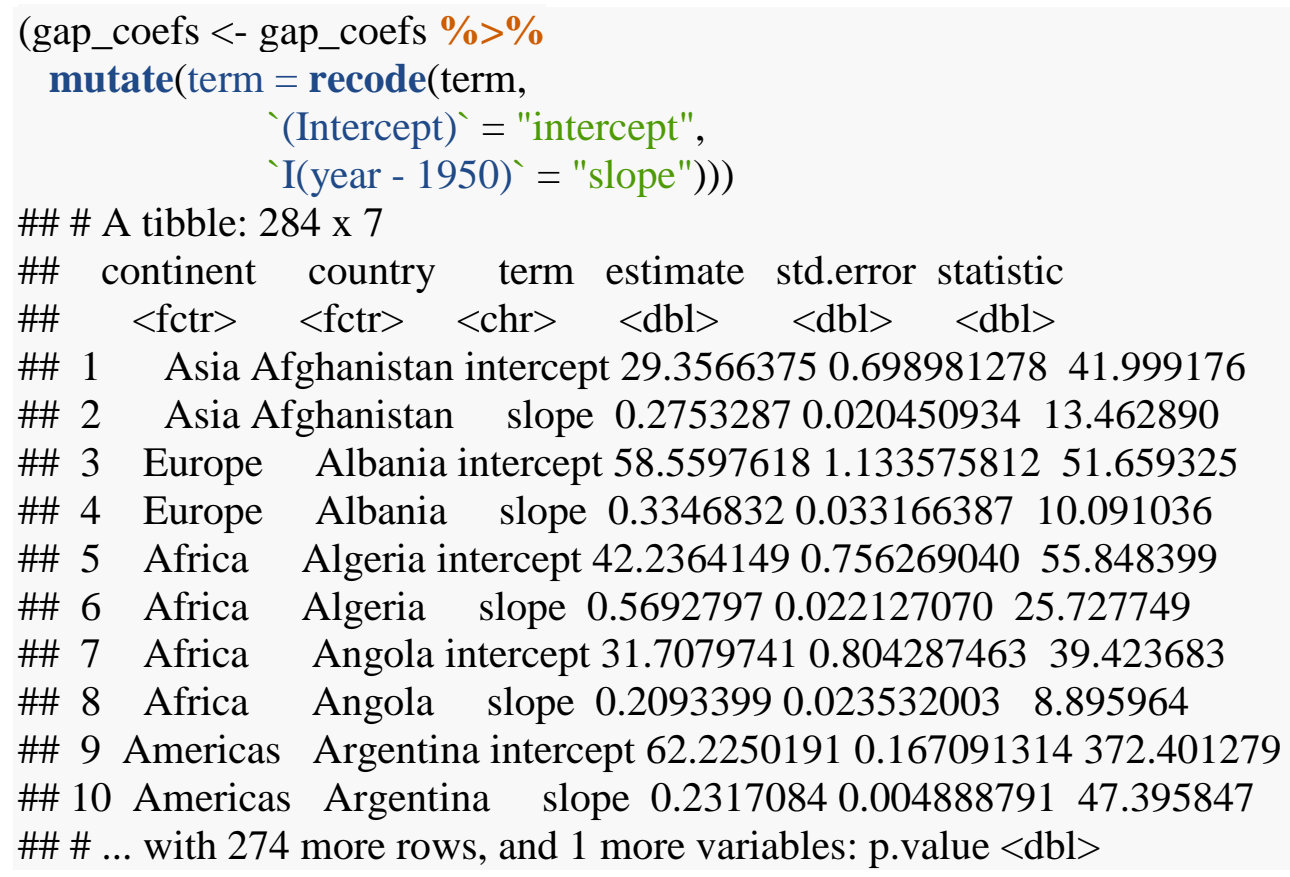

Due to the way we parametrized the model, the intercepts correspond to expected life expectancy in 1950. These numbers should be plausible as human life expectancies. The slopes correspond to change in expected life expectancy from one year to the next. We expect positive numbers to dominate, but they'll probably be less than one. A reshaped version of the estimates, gap_ests, is handy for numerical summarization and visualization.

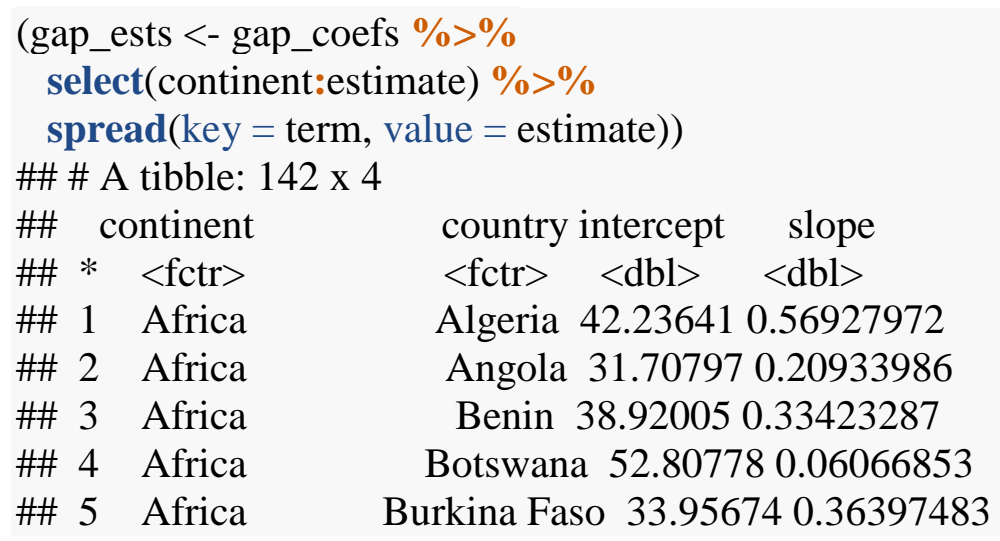




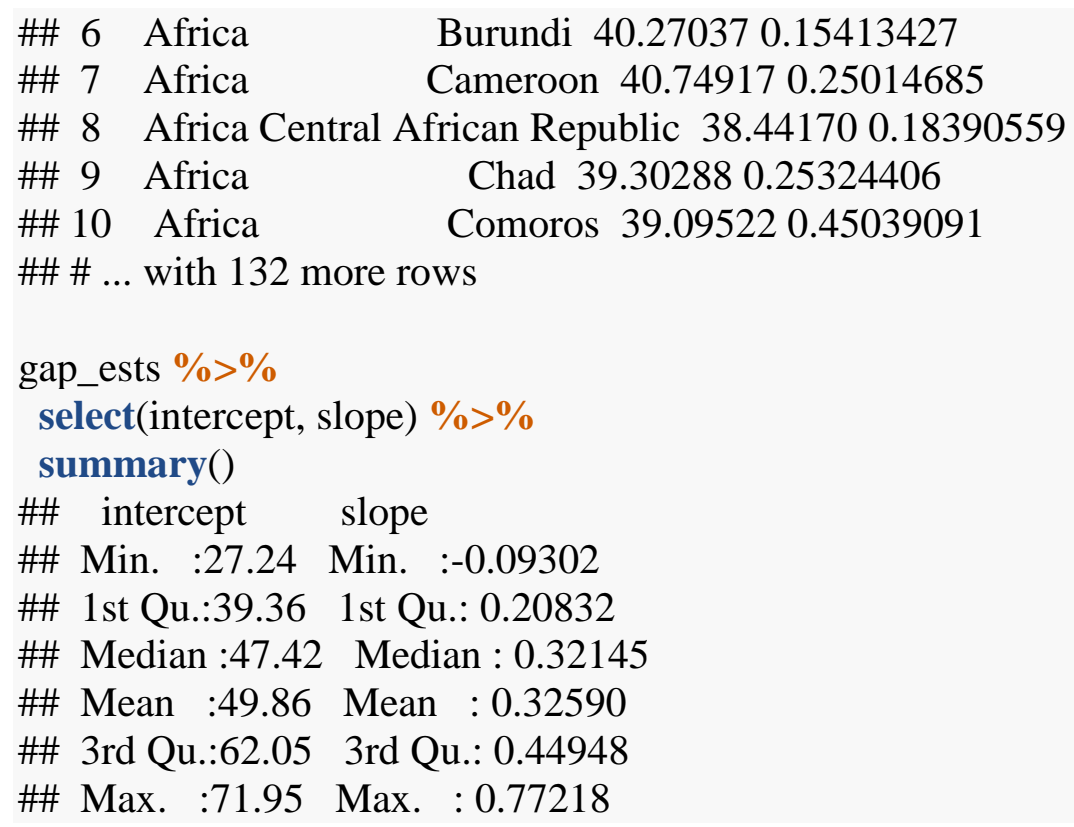

The numerical summaries look reasonable. We conclude with a look at the full distribution.

ggplot(gap_coefs, aes $(\mathrm{x}=$ estimate $))+$ geom_density ()$+$ geom_rug( $)+$ facet_wrap $(\sim$ term, scales = "free" $)$ ggplot(gap_ests, aes $(\mathrm{x}=$ intercept, $\mathrm{y}=$ slope $))+$ geom_point ()$+$ geom_smooth(se = FALSE, lwd = 2) 

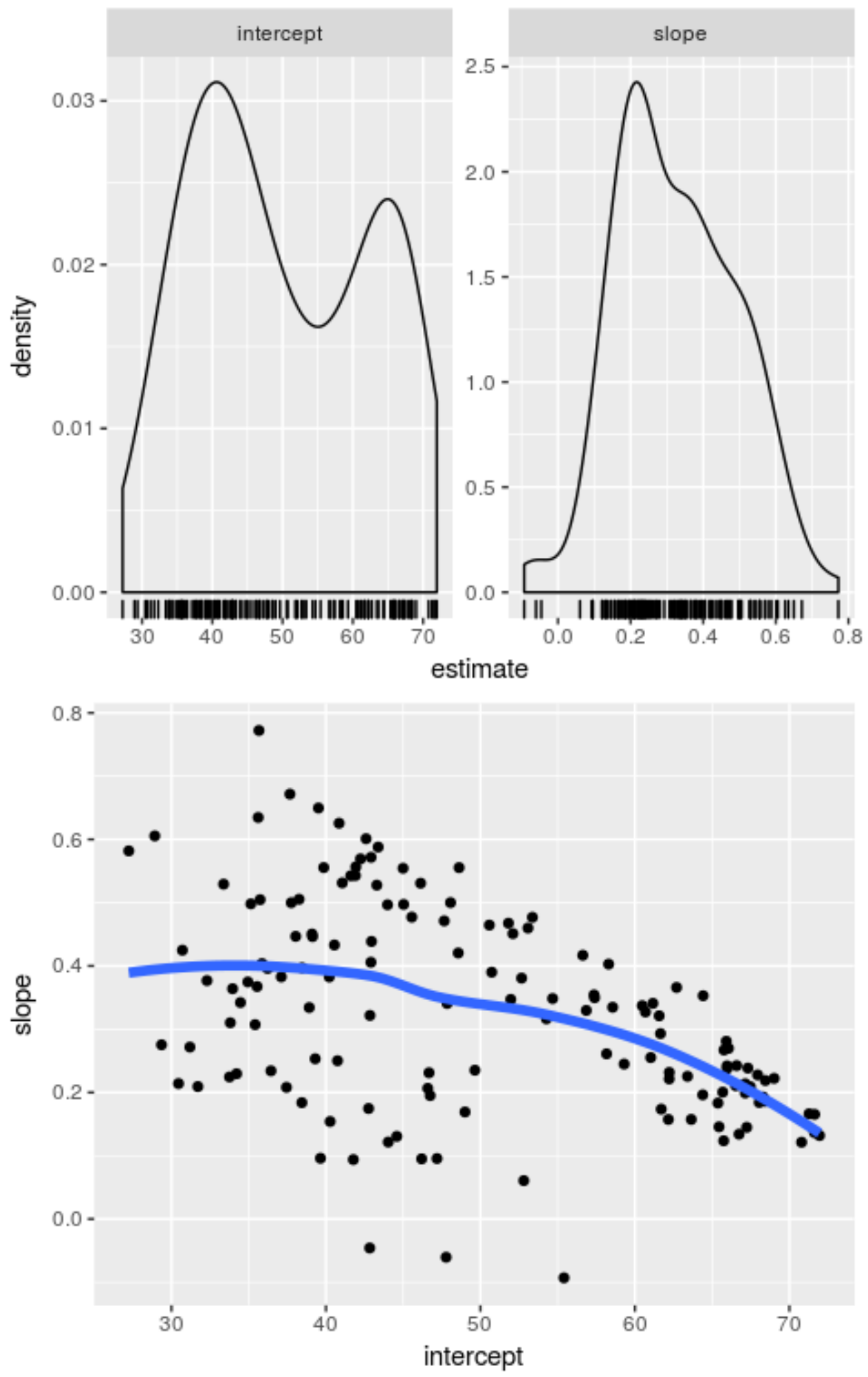

Figure 12.1 Distributions of model intercepts and slopes and their relationship in a scatter plot 


\subsection{EXERCISE}

1. Fit linear regression models of the daily bike counts on precipitation and max temperature, first for both bridges together and then for each bridge separately using the split-applycombine pattern.

2. When using ggfortify to plot weekly variation, trend and noise separately, you need to plot each bridge separately (sample code here). Use the split-apply-combine to avoid having to repeat for each bridge.

\subsection{RESOURCES:}

1. purrr package

2. purrr tutorial

3. Software Carpentry lesson on Split-Apply-Combine 


\subsection{WHERE TO GO FROM HERE}

Practice makes perfection. Use what you learned here as much as possible in your own research and work, even though you may not master everything first off. Once you find a solution to your data science problem, think about whether it conforms to the best practices for data science. If not, can you improve your solution according to the best practices?

\subsection{NOTICEABLE FEATURES NOT COVERED}

$\mathrm{R}$ is getting more powerful all the time. There are many exciting features and packages that we have not covered and much more are emerging. Below are a few features I think are worth mentioning.

\subsubsection{Automate Testing Your Code}

It is always a good idea to test your code with simple inputs to make sure it works correctly before adding complexity and deploying it for actual work (see best practices for data science 5). There are ways to automate the tests so you will not forget to run them. Hadley Wickham writes about testing for $\mathrm{R}$ packages and testthat is an $\mathrm{R}$ package specifically to make testing. And if you use GitHub or other popular public version control websites, it is possible to use Travis-CI to do "continuous integration" tests for your code. Julia Silge has a nice tutorial for beginners.

\subsubsection{Automate Your Workflow}

If you are a seasoned programmer and are familiar with the make toolchain, you can use it to automate your workflow too. Professor Jenny Bryan provides a tutorial for her STAT 545 class. A more research-oriented solution is to use the remake package:

The idea here is to re-imagine a set of ideas from make but built for R. Rather than having a series of calls to different instances of $\mathrm{R}$ (as happens if you run make on $\mathrm{R}$ scripts), the idea is to define pieces of a pipeline within an $\mathrm{R}$ session. Rather than being language agnostic (like make must be), remake is unapologetically $\mathrm{R}$ focused.

remake is still under heavy development and is not yet available on CRAN. You will have to install it from GitHub:

library(devtools) install_github("richfitz/remake")

\subsubsection{Develop Interactive Apps With Shiny}

Shiny allows you to develop interactive apps that run on top of R. Again, Professor Jenny Bryan has a nice tutorial for getting started with Shiny. 


\subsection{WHERE TO FIND HELP}

1. Help documents;

2. Package vignettes and manual;

3. R cookbook;

4. Stack Overflow and Cross Validated, A Q\&A community for programming and statistics;

5. Google is your friend; and

6. Ask a person who may know. 


\section{REFERENCES}

Merali, Zeeya. 2010. “Computational Science: ...Error.” Nature News 467 (7317): 775-77. doi:10.1038/467775a.

University Transportation Research Center. 2014. “Ground Transportation Technology Symposium: Big Data and Innovative Solutions for Safe, Efficient and Sustainable Mobility.” In. New York, NY.

Wilson, Greg, D. A. Aruliah, C. Titus Brown, Neil P. Chue Hong, Matt Davis, Richard T. Guy, Steven H. D. Haddock, et al. 2014. "Best Practices for Scientific Computing.” PLOS Biology 12 (1): e1001745. doi:10.1371/journal.pbio.1001745. 
Transportation Research and Education Center

Portland State University

1900 S.W. Fourth Ave., Suite 175

Portland, OR 97201 